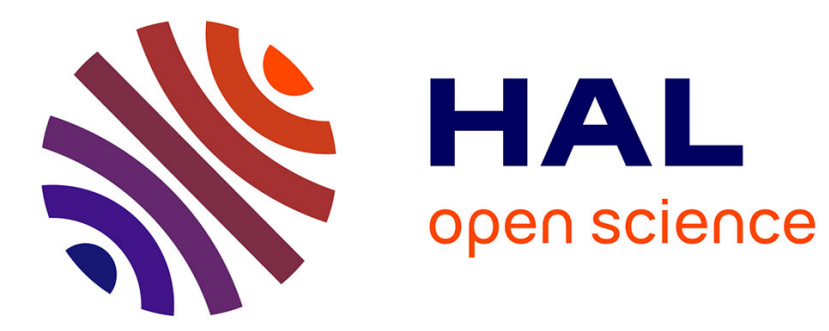

\title{
Quarante années de mobilité sociale en France: L'évolution de la fluidité sociale à la lumière de modèles récents
}

\author{
Louis-André Vallet
}

\section{- To cite this version:}

Louis-André Vallet. Quarante années de mobilité sociale en France : L'évolution de la fluidité sociale à la lumière de modèles récents. Revue française de sociologie, 1999, 40 (1), pp.5-64. 10.2307/3322517 . hal-03455225

\section{HAL Id: hal-03455225 \\ https://hal.science/hal-03455225}

Submitted on 29 Nov 2021

HAL is a multi-disciplinary open access archive for the deposit and dissemination of scientific research documents, whether they are published or not. The documents may come from teaching and research institutions in France or abroad, or from public or private research centers.
L'archive ouverte pluridisciplinaire HAL, est destinée au dépôt et à la diffusion de documents scientifiques de niveau recherche, publiés ou non, émanant des établissements d'enseignement et de recherche français ou étrangers, des laboratoires publics ou privés. 


\section{Quarante années de mobilité sociale en France. L'évolution de la} fluidité sociale à la lumière de modèles récents

Louis-André Vallet

\section{Citer ce document / Cite this document :}

Vallet Louis-André. Quarante années de mobilité sociale en France. L'évolution de la fluidité sociale à la lumière de modèles récents. In: Revue française de sociologie, 1999, 40-1. Fluidité et hiérarchie. L'évolution de la stratification sociale en France. pp. 5-64;

doi : $10.2307 / 3322517$

https://www.persee.fr/doc/rfsoc_0035-2969_1999_num_40_1_5146

Fichier pdf généré le 23/04/2018 


\section{Resumen}

Louis-André Vallet : Cuarenta años de mobilidad social en Francia. La evolución de la fluidéz social a la vista de los modelos recientes.

Este artículo trata de hacer conocer si una tendencia en el regimen de movilidad social de la sociedad francesa puede ser identificada a partir de la mitad del siglo a largo plazo. Comienza por una revisión de la literatura internacional sobre las tendencias temporales de la fluidéz social dentro de las sociedades modernas. Analizando las últimas investigaciones francesas que han concluído a una estabilidad de la desiguladad de las oportunidades sociales en las dos últimas décadas en este país, se argumenta que esta conclusión podría ser una consecuencia de la falta de rigor de las técnicas estadísticas utilizadas. A continuación el artículo analiza los cuadros de movilidad social padre-hijo y padre-hija, extraídas de encuestas nacionales representativas efectuadas en 1953, 1970, 1977, 1985 y 1993 ( $N=35741$ para los hombres y 18484 para las mujeres). La utilización de los modelos loglineales y de los modelos log-multiplicativos revela que la asociación estadística (medida por el logaritmo de los odds ratios) entre origen y posición social en esos cuarenta años ha disminuído a un ritmo anual de 0,5\%. A partir de mediados de este siglo este resultado corresponde a una tendencia lenta, pero contínua de la reducción de la desigualdad de las oportunidades sociales. De los doce millones de franceses, hombres y mujeres, entre 35 y 59 años con empleo en 1993, cerca de medio milión ocupan de este modo posiciones sociales que no habrían sido las suyas sin la presencia de este incremento de la fluidéz social en los últimos cuarenta años. El artículo concluye con la tesis de la invariabilidad temporal del regimen de la movilidad entre generaciones es insostenible para la sociedad francesa y que las causas de esta evolución quedan sin precisar.

\section{Zusammenfassung}

Louis-André Vallet: Vierzig Jahre der sozialen Mobilität in Frankreich. Die Entwicklung der sozialen Durchlässigkeit im Licht von kürzlichen Modellen.

Dieser Artikel beschäftigt sich mit der Frage, ob im Regim der sozialen Mobilität der französischen Gesellschaft seit Mitte dieses Jahrhunderts eine Langzeittendenz festgestellt werden kann. Er beginnt mit einer Überschau der internationalen Literatur zu den zeitlichen Tendenzen der sozialen Durchlässigkeit innerhalb der modernen Gesellschaften. Durch eine Analyse der kürzlichen französischen Forschungen, die auf die Stabilität der Ungleichheit der sozialen Chancen in Frankreich über die letzten zwanzig Jahre schliesst, argumentiert der Aufsatz, dass diese Schlussfolgerung lediglich mit zu schwachen angewendeten statistischen Techniken zusammenhangen könnte. Der weitere Verlauf des Artikels beschäftigt sich mit einer Analyse der Tabellen der sozialen Mobilität Vater-Sohn und Vater-Tochter, die aus repräsentativen nationalen Umfragen aus den Jahren 1953, 1970, 1977, 1985 und 1993 ( $N=35741$ für die Männer und 18484 für die Frauen). Die Verwendung von log-linearen und log-multiplikativen Modellen zeigt, dass die statistische Verbindung (gemessen mit dem Logarithmus der odds ratios) zwischen sozialen Herkunft und sozialen Stellung regelmassig um $0,5 \%$ pro Jahr in den letzten vierzig Jahren abgenommen hat. Dieses Ergebnis entspricht einer langsamen jedoch andauernden Tendenz zur Verminderung der Ungleichheit der sozialen Chancen seit der Mitte dieses Jahrhunderts. Von den zwölf Millionen Franzosen, Männer und Frauen, zwischen 35 und 59 Jahre, die 1993 eine Beschäftigung hatten, nimmt fast eine halbe Million soziale Stellungen ein, die sie nicht gehabt hätten, wenn die soziale Durchlässigkeit der letzten vierzig Jahren nicht zugenommen hätte. Der Aufsatz schliesst mit der Feststellung, dass die These der zeitlichen Unveränderlichkeit des Regims der Mobilität zwischen Generationen für die französische Gesellschaft nicht aufgestellt werden kann und, dass die Gründe zu dieser Entwicklung noch festgelegt werden müssen.

\section{Abstract}

Louis-André Vallet : Forty years of social mobility in France. Temporal trends in social fluidity illuminated by recent models.

The aim of this paper is to examine whether a long-term trend can be identified in the mobility regime 
of French society from the middle of the century. Its begins with a review of the international literature on temporal trends in social fluidity within modern societies. Analysing recent French research which has concluded that inequality of opportunity has remained unchanged in France during the last two decades, the paper argues that such a conclusion can only have resulted from the use of insufficiently powerful statistical techniques. The second part of the paper analyses father-son and father-daughter mobility tables drawn from national representative surveys carried out in 1953, 1970, 1977, 1985 and 1993 ( $N=35,741$ for males and 18, 484 for females). The use of log-linear and log-multiplicative models reveals that the statistical association (as measured with the logarithm of the odds ratio) between social origin and destination has declined steadily by $0.5 \%$ a year over a period of forty years. This finding highlights a slow but continuous trend towards a reduction in inequality of opportunity from the middle of the century. Of the twelve million French men and women between the ages of 35 and 59 who were in employment in 1993, nearly half a million would have belonged to different classes without this forty-year increase in social fluidity. The paper concludes that the thesis of temporal invariance in the intergenerational mobility regime cannot be maintained for France, but that the reasons of this change still remain to be ascertained.

\section{Résumé}

Cet article traite la question de savoir si une tendance de long terme peut être identifiée dans le régime de mobilité sociale de la société française depuis le milieu du siècle. Il s'ouvre par une revue de la littérature internationale sur les tendances temporelles de la fluidité sociale au sein des sociétés modernes. Analysant les recherches françaises récentes qui ont conclu à la stabilité de l'inégalité des chances sociales dans la France des deux dernières décennies, il argumente que cette conclusion pourrait n'être liée qu'à un manque de puissance des techniques statistiques mises en œuvre. La suite de l'article est consacrée à une analyse des tables de mobilité sociale père-fils et père-fille tirées d'enquêtes nationales représentatives conduites en 1953, 1970, 1977, 1985 et 1993 ( $N=35741$ pour les hommes et 18484 pour les femmes). L'usage de modèles log-linéaires et log-multiplicatifs révèle que l'association statistique (mesurée par le logarithme des odds ratios) entre origine et position sociales a diminué au rythme régulier de 0,5\% par an durant quarante ans. Ce résultat correspond à une tendance lente, mais continue, à la réduction de l'inégalité des chances sociales depuis le milieu du siècle. Parmi les douze millions de Français, hommes et femmes, de 35 à 59 ans qui ont un emploi en 1993, près d'un demi-million occupent ainsi des positions sociales qui n'auraient pas été les leurs en l'absence de cette augmentation de la fluidité sociale en quarante ans. L'article conclut que la thèse de l'invariance temporelle du régime de mobilité entre générations ne peut être soutenue pour la société française et que les causes de cette évolution restent à préciser. 


\title{
Quarante années de mobilité sociale en France
}

\author{
L'évolution de la fluidité sociale à la lumière \\ de modèles récents*
}

\begin{abstract}
RÉSUMÉ
Cet article traite la question de savoir si une tendance de long terme peut être identifiée dans le régime de mobilité sociale de la société française depuis le milieu du siècle. Il s'ouvre par une revue de la littérature internationale sur les tendances temporelles de la fluidité sociale au sein des sociétés modernes. Analysant les recherches françaises récentes qui ont conclu à la stabilité de l'inégalité des chances sociales dans la France des deux dernières décennies, il argumente que cette conclusion pourrait n'être liée qu'à un manque de puissance des techniques statistiques mises en œuvre. La suite de l'article est consacrée à une analyse des tables de mobilité sociale père-fils et père-fille tirées d'enquêtes nationales représentatives conduites en $1953,1970,1977,1985$ et $1993(\mathrm{~N}=35741$ pour les hommes et 18484 pour les femmes). L'usage de modèles log-linéaires et log-multiplicatifs révèle que l'association statistique (mesurée par le logarithme des odds ratios) entre origine et position sociales a diminué au rythme régulier de $0,5 \%$ par an durant quarante ans. Ce résultat correspond à une tendance lente, mais continue, à la réduction de l'inégalité des chances sociales depuis le milieu du siècle. Parmi les douze millions de Français, hommes et femmes, de 35 à 59 ans qui ont un emploi en 1993, près d'un demi-million occupent ainsi des positions sociales qui n'auraient pas été les leurs en l'absence de cette augmentation de la fluidité sociale en quarante ans. $L$ 'article conclut que la thèse de l'invariance temporelle du régime de mobilité entre générations ne peut être soutenue pour la société française et que les causes de cette évolution restent à préciser.
\end{abstract}

Est-il possible d'identifier des tendances temporelles de long terme dans le régime de mobilité sociale des sociétés modernes? Cette question reste, aujourd'hui comme hier, l'une des plus débattues parmi les sociologues de la stratification et de la mobilité. En 1927, Pitirim Sorokin y répondait négativement en émettant un doute quant à la possibilité de discerner une tendance

* Nous remercions le LASMAS - Institut du Longitudinal (CNRS) et le Laboratoire de Sociologie Quantitative (CREST - INSEE) qui ont mis à notre disposition les données des enquêtes utilisées. Nous remercions aussi Raymond Boudon, Louis Chauvel, John H. Goldthorpe, Michel Gollac, Dominique Merllié, Claude Thélot ainsi que nos collègues de la Revue française de sociologie de leurs commentaires sur la version initiale de ce texte. Les modèles utilisés dans cet article ont été estimés à l'aide du logiciel $\ell_{E M}$ (version 1.0 du 18 septembre 1997) développé par Jeroen K. Vermunt (Université de Tilburg). Voir aussi, du même auteur, l'ouvrage Log-linear models for event histories (1997). 
séculaire dans l'évolution de la mobilité au sein d'une société : «Dans le domaine de la mobilité verticale [...] il semble n'exister aucune tendance perpétuelle bien déterminée à l'accroissement ou bien à la diminution de l'intensité et du caractère général de la mobilité. Nous proposons cette thèse comme étant valide pour l'histoire d'un pays, pour celle d'un large corps social et, en définitive, pour l'histoire de l'humanité. » (1959, p. 152). Le même auteur reconnaissait toutefois que de nombreuses tendances avaient pu exister au cours de l'histoire et qu'en particulier, au sein des sociétés occidentales, l'hérédité des professions avait décru durant le dernier siècle. Mais, sur une longue période, Sorokin voyait les vagues de plus forte mobilité remplacées par les cycles de plus grande immobilité et il concluait ainsi, sur le très long terme, à l'existence de «fluctuations sans tendance».

Dans la sociologie contemporaine, la thèse de Sorokin qui portait sur les taux de mobilité absolus - c'est-à-dire sur la mobilité observée - a, dans une large mesure, trouvé sa continuation dans l'hypothèse plus subtile d'invariance temporelle du régime de mobilité formulée en 1975 par Hauser, Koffel, Travis et Dickinson. Selon cette thèse, l'existence de variations temporelles significatives dans les taux observés de mobilité ne serait due qu'à des transformations macrostructurelles - notamment l'évolution de la distribution des emplois indépendantes de la structure profonde de la mobilité alors que les taux de mobilité relatifs demeureraient stables dans le temps. En d'autres termes, l'évolution de la structure socioprofessionnelle au fil des générations conduirait à ce que les chances qu'ont les hommes d'une origine sociale donnée d'accéder à l'une plutôt qu'à l'autre de deux positions sociales se modifieraient au cours du temps ; en revanche, comparées aux chances correspondantes que connaissent les hommes d'une seconde origine sociale, celles des premiers demeureraient inchangées. Le bouleversement phénotypique de la mobilité observée irait donc de pair avec la permanence génotypique du niveau d'association statistique entre origine et position sociales. C'est précisément ce qu'observaient Hauser et al. (1975) sur les données de mobilité américaines disponibles en tirant profit des méthodes de modélisation log-linéaire récemment introduites en sociologie par Goodman (1972). Popularisée dans l'ouvrage de Goldthorpe (1980) sous le nom de fluidité sociale constante, la même hypothèse a été confrontée, souvent avec succès, aux données empiriques recueillies dans de nombreux pays, conduisant ainsi à conclure à la forte inertie des régimes de mobilité sociale entre générations (voir par exemple Goldthorpe, Payne, 1986). Le titre de l'ouvrage comparatif qu'Erikson et Goldthorpe (1992) ont consacré à la mobilité sociale dans les sociétés industrielles - The constant flux - porte ainsi la marque de la puissance descriptive de cette thèse d'invariance temporelle de la fluidité sociale. Elle conduit à supposer qu'un niveau fixe d'inégalité des chances sociales est inscrit au cœur de la structure des sociétés modernes.

«Fluctuations sans tendance» des taux absolus et invariance temporelle des taux relatifs ne sont pas les seules hypothèses énoncées par les sociologues à propos de l'évolution de la mobilité sociale. Dans la lignée des travaux de Parsons (1951) et de Kerr et al. (1960) sur les exigences fonctionnelles qu'impose le processus d'industrialisation, des sociologues nord-américains ont dé- 
veloppé une argumentation conduisant à anticiper une croissance lente, mais continue, du niveau général de la fluidité sociale caractéristique des sociétés modernes. Dans The American occupational structure (1967, p. 429), Blau et Duncan énoncent ainsi que le changement technologique a redéfini les conditions du recrutement dans les professions, au sein de l'économic américaine. Selon ces auteurs, une tendance fondamentale vers un universalisme croissant caractérise la société industrielle : cette dernière serait sous l'emprise grandissante de critères objectifs d'évaluation universellement acceptés. Blau et Duncan s'attendent ainsi à ce que les statuts supérieurs puissent de moins en moins être directement hérités et que leur possession doive de plus en plus être légitimée par la preuve de compétences personnelles particulières. Ils avancent donc que les sociétés modernes se caractériseraient par moins d'ascription c'est-à-dire moins d'importance et de poids accordés aux aspects hérités de la famille d'origine - et plus d'achievement - plus d'importance et de poids accordés au statut acquis, par le biais de l'éducation en premier lieu. Étudiant, à partir de l'enquête Occupational changes in a generation de 1962 et au sein de comparaisons intercohortes, les déterminants du niveau d'éducation atteint et du statut du premier emploi exercé, Blau et Duncan n'ont cependant décelé aucune tendance temporelle claire. Trois années plus tard, Treiman (1970) a aussi avancé l'idée d'une relation directe entre niveau de développement économique et mobilité sociale et a énoncé la thèse de l'industrialisme selon laquelle le niveau de transfert direct de statut, des parents aux enfants, irait en diminuant avec le processus d'industrialisation d'une société. Celui-ci transformerait en effet, non seulement les institutions économiques comme la structure du marché du travail, mais aussi les institutions sociales telles que la famille et le système éducatif. Directement ou indirectement, l'industrialisation mettrait à bas les anciennes barrières, ouvrirait de nouvelles possibilités d'ascension sociale et déplacerait les bases de l'acquisition du statut de l'ascription à l'achievement. Outre les transformations de la distribution socioprofessionnelle qu'elle impose et la mobilité structurelle qu'elle implique, l'industrialisation desserrerait donc le niveau de dépendance des destinées individuelles à l'égard des milieux d'origine et engendrerait ainsi une ouverture graduelle du régime de mobilité des sociétés. En plus de retenir l'attention des sociologues, cette thèse a aussi fait l'objet de recherches d'histoire sociale au cours des deux dernières décennies (voir notamment Kaelble, 1981 ; Miles, Vincent, 1993 ; Van Leeuwen, Maas, 1996).

\section{Un débat international sur l'évolution temporelle de la fluidité sociale}

Dans une recherche ambitieuse, Ganzeboom, Luijkx et Treiman (1989) réexaminent la question des variations nationales et temporelles du régime de mobilité sociale entre générations. Ils utilisent à cette fin un ensemble de 149 tables de mobilité provenant de 35 pays, toutes recueillies, à l'exception de cinq d'entre elles, à partir de 1955 et présentées dans la même nomenclature en six classes. Pour décrire, au sein de chaque table de mobilité, la force et la structure 
du lien entre origine et position sociales, les auteurs mettent en œuvre un modèle général d'analyse dû à Goodman (1979) et qui représente cette association statistique par trois ensembles de paramètres : deux échelles de scores estimés, relatives l'une, aux classes d'origine, l'autre, aux classes de destination; un paramètre unique qui mesure la force générale de l'association intrinsèque entre origine et position (1). Ganzeboom, Luijkx et Treiman conduisent leur étude des variations sociétales et temporelles de la fluidité sociale en deux étapes. En premier lieu, ils sélectionnent deux modèles statistiques qui mesurent les différences de régime de mobilité sous une forme parcimonieuse. Pour chaque table est ainsi estimé un paramètre général pour la force du lien entre la classe sociale du père et celle du fils et, éventuellement, un second paramètre exprimant la tendance d'ensemble à l'hérédité sociale. Considérant alors chaque table de mobilité comme une observation particulière, les auteurs se demandent ensuite dans quelle mesure les variations de ces paramètres dépendent et peuvent être expliquées par le pays considéré, l'année de l'enquête, les écarts existant dans la qualité des données et les erreurs de mesure.

Selon les analyses effectuées, un cinquième de la variance du paramètre général d'association provient des différences de qualité entre les données, mais un tiers supplémentaire est à porter au compte de la variable «pays». Ganzeboom et al. en déduisent que si les nations sont assez semblables dans la forme générale de la fluidité sociale, elles diffèrent nettement dans le niveau de celle-ci. Enfin, une fraction modeste-2 à $3 \%$ - mais significative de la variance renvoie aux périodes où les données ont été recueillies. Toutes les estimations produites par les auteurs sont ici convergentes. Elles conduisent à conclure que la force du lien entre origine et position sociales décroît au rythme faible, mais régulier, d'environ un pour cent par an et Ganzeboom, Luijkx et Treiman commentent leur analyse en ces termes : «Ce résultat fournit un support empirique important en faveur de l'idée qu'il existerait une tendance séculaire mondiale à l'augmentation de la fluidité sociale [...]. La décroissance du paramètre général d'association est d'environ un pour cent par an. Bien qu'il s'agisse, à court terme, d'une quantité négligeable (et par conséquent difficile à estimer sur des périodes brèves), elle implique un changement très substantiel à long terme.» (1989, pp. 44-45).

Pour confirmer leur découverte d'une tendance séculaire mondiale à l'ouverture des sociétés (2), Ganzeboom et al. réanalysent de manière séparée les données relatives aux pays pour lesquels ils disposent d'au moins trois tables de mobilité de qualité suffisante. Il apparaît alors que, pour quinze des dix-huit

(1) Connu sous le nom de «modèle (quasi) log-multiplicatif à effets lignes et colonnes », ce modèle incorpore en outre six paramètres diagonaux pour capter la tendance, spécifique à chaque classe, à l'hérédité sociale entre générations. Nous en donnerons une présentation plus formalisée dans l'avant-dernière section de cet article où nous l'utiliserons, à titre de confirmation de nos résultats, sur les données françaises. Voir aussi Clogg et Shihadeh (1994) pour une présentation générale de ce modèle - qui ne nécessite pas d'hypothèse a priori sur l'ordre des catégories dans la structure sociale - ainsi que de ses extensions.

(2) Nous traduisons ainsi l'expression «our discovery of a world wide secular trend toward increased societal openness » qu'utilisent les auteurs (1989, p. 45). 
pays concernés, le coefficient estimé est de signe négatif, traduisant ainsi une tendance linéaire quasi générale à la décroissance de l'association statistique entre origine et position sociales (3) et les auteurs calculent enfin qu'un tel résultat - ou un autre encore plus extrême - n'aurait que trois chances sur mille d'advenir sous l'hypothèse nulle d'absence de transformation temporelle.

En retenant l'attention de la communauté des sociologues, la recherche de Ganzeboom, Luijkx et Treiman a aussi fait l'objet de plusieurs critiques. Jones (1992) a, en premier lieu, mis en lumière le fait que les auteurs n'avaient pas testé l'hypothèse d'une fluidité sociale commune aux 149 tables étudiées. Réanalysant leurs données, il montre alors que cette hypothèse rend compte de $95 \%$ de l'association statistique expliquée par le modèle que Ganzeboom et al. préfèrent, les $5 \%$ restant tenant alors aux variations des régimes de mobilité, entre pays et au cours du temps. Conclure, comme le font Ganzeboom, Luijkx et Treiman (1989, p. 48), que l' " hypothèse de fluidité sociale commune est tout simplement incorrecte » apparaît alors nettement exagéré.

Dans le chapitre qu'ils consacrent aux tendances temporelles de la mobilité sociale, Erikson et Goldthorpe passent aussi au crible la même recherche (1992, pp. 99-101). Ils soulignent tout d'abord l'inconvénient qu'il y a à traiter avec la même importance et dans une analyse unique des données de qualité et de robustesse très inégales. Par exemple, si Ganzcboom, Luijkx et Treiman utilisent, pour la France, les enquêtes Formation - Qualification Professionnelle de 1964 et 1970, leurs tables de mobilité de 1958 et 1967 ne portent respectivement que sur 335 et 743 individus. Erikson et Goldthorpe relèvent ensuite un aspect des résultats de Ganzeboom et al. qui s'accorde mal avec leur conclusion générale : le fait que, si la décroissance du lien entre classe sociale du père et classe sociale du fils est significative pour le paramètre général d'association, il n'en va pas de même pour la tendance d'ensemble à l'hérédité sociale. Une troisième critique met en avant le caractère restrictif du modèle que les auteurs préfèrent : estimant une échelle unique pour les classes d'origine et les classes de destination, il ne s'ajuste en réalité correctement qu'à une minorité des tables de mobilité rassemblées. Rejoignant la critique de Jones, Erikson et Goldthorpe montrent pour finir que le respect des critères de sélection de modèles que Ganzeboom, Luijkx et Treiman mettent en œuvre aurait dû en fait les conduire à mettre en avant l'hy pothèse d'invariance nationale et temporelle de la fluidité sociale.

C'est enfin Wong (1994) qui s'est livré à une analyse secondaire soigneuse des mêmes données, et tout particulièrement de celles relatives aux États-Unis entre 1947 et 1986, à l'Angleterre (1951-1986) et au Japon (1955-1975). Contrairement à la stratégie adoptée par Ganzeboom, Luijkx et Treiman, les versions différentes d'un même modèle sont ici confrontées de manière systématique - estimations sous hypothèse d'invariance temporelle, sous hypothèse

(3) Cette tendance linéaire n'est toutefois significative que pour neuf ou douze pays (selon le modèle considéré) dont la France pour laquelle les tables de mobilité utilisées ont été recueillies en $1958,1964,1967$ et 1970. 
de changements quelconques d'une date à une autre, sous hypothèse de tendance linéaire enfin. Les résultats ainsi obtenus remettent en cause la conclusion principale de Ganzeboom et al. : l'évolution temporelle de la fluidité sociale est souvent insignifiante et difficile à mettre en évidence. Parmi les dix-huit pays étudiés, la conclusion irréfutable d'une ouverture progressive du régime de mobilité entre générations ne peut être établie que pour la Hongrie et la Suède ; elle est moins assurée s'agissant de l'Angleterre, des États-Unis, de la France et des Pays-Bas; dans les autres pays, les analyses statistiques confortent l'hypothèse d'une très grande stabilité du régime de mobilité. Dans sa conclusion, Wong est ainsi conduit à exprimer ses doutes quant à l'existence d'une tendance universelle à l'ouverture des sociétés et renvoie l'explication des changements temporels de fluidité sociale à la prise en compte des contextes historiques nationaux.

Pour tester les prédictions de la «théorie libérale de l'industrialisme »-selon l'expression qu'ils emploient -, Erikson et Goldthorpe (1992, pp. 86-96) mettent en ouvre une méthodologie très différente de celle de Ganzeboom, Luijkx et Treiman. Disposant, pour neuf pays européens, d'une enquête nationale représentative conduite dans la première moitié des années soixante-dix et dont les données ont été recodées dans une nomenclature homogène de classes, ils analysent les tendances de la mobilité sociale en confrontant les expériences d'hommes appartenant à quatre cohortes de naissance, i.e. âgés de 25-34 ans, 35-44 ans, 45-54 ans ou 55-64 ans au moment de l'enquête. Erikson el Goldthorpe estiment en premier lieu un modèle log-linéaire qui postule l'identité des taux relatifs de mobilité entre les cohortes. Dans huit des neuf pays étudiés, un tel modèle rend compte de plus de $90 \%$ de l'association totale observée entre origine et position sociales; dans aucun d'eux, il ne classe de manière erronée plus de $5 \%$ de l'effectif total considéré. Ces résultats apportent de l'cau au moulin de l'hypothèse de stabilité temporelle des régimes de mobilité; comme l'indiquent les auteurs, ils suggèrent en effet que si des transformations sont intervenues dans l'ouverture des sociétés, elles ne sont en aucun cas très substantielles. Erikson et Goldthorpe reconnaissent toutefois immédiatement l'insuffisance d'un tel test empirique : «[Le test que nous avons conduit] a un caractère très général ou "global" et n'est doté par conséquent que d'une faible puissance pour détecter des évolutions qui pourraient avoir pris place dans des aspects plus particuliers des régimes de mobilité. En d'autres termes, même là où l'hypothèse représentée par le modèle de fluidité sociale constante ne peut à coup sûr être rejetée, la possibilité demeure encore que certains changements soient advenus au fil des cohortes qui, tout en étant globalement modestes, pourraient néanmoins avoir un intérêt réel. Et, à ce propos, nous devons clairement reconnaître que la théoric libérale n'affirme pas seulement que le pattern de fluidité sociale se transforme, mais qu'il le fait dans une direction particulière, c'est-à-dire vers une fluidité accrue. Ainsi, si nous devons rendre justice à cette théoric, il nous faut tester aussi précisément que nous le pouvons l'existence de tendances de cette sorte.»(1992, pp. 90-91).

Pour avancer dans cette voie, Erikson et Goldthorpe envisagent deux modifications du modèle d'invariance temporelle. La première consiste à supposer 
que la forme générale de la fluidité sociale est stable au cours du temps, mais que son niveau varie systématiquement d'une cohorte à une autre. En retenant comme référence l'une des cohortes de naissance, l'estimation, pour chacune des autres, d'un paramètre supplémentaire traduit alors le fait que le régime de mobilité qui la caractérise est uniformément plus proche (ou plus éloigné) de la situation d'égalité des chances sociales que représente l'absence complète de lien entre classe d'origine et classe de destination (4). La seconde modification qu'Erikson et Goldthorpe envisagent porte sur l'immobilité. Elle consiste à postuler la stabilité temporelle du régime de mobilité, mis à part le fait que la tendance intrinsèque à «hériter» de la position paternelle varierait, pour toutes les classes, d'une cohorte à une autre. Dans ce cadre, la théorie générale de l'industrialisme conduit à attendre un accroissement du niveau général de la fluidité et/ou une diminution de la propension à l'hérédité sociale, des cohortes de naissance les plus anciennes aux plus récentes.

Le résultat extrême où les deux modifications envisagées s'accompagneraient d'une amélioration de la qualité de l'ajustement et exprimeraient une variation monotone, à la fois dans l'accroissement du niveau général de la fluidité sociale et dans l'affaiblissement de la propension à l'immobilité, n'apparaît dans aucun des pays étudiés. C'est la société hongroise qui est la plus proche d'une telle situation en manifestant une tendance continue à l'égalisation des chances sociales pour les trois cohortes les plus anciennes, mais non la plus récente. Dans trois autres pays, le modèle de différence uniforme est significativement plus proche des données que celui d'invariance temporelle : les paramètres estimés pour la République fédérale d'Allemagne et la Suède ne mettent toutefois au jour aucune variation temporelle régulière ; ceux relatifs à la France font en revanche apparaître une tendance à la diminution de la fluidité sociale, des plus âgés aux plus jeunes (5). Dans les cinq pays restant, outre le fait qu'aucune des modifications apportées au modèle d'invariance temporelle n'est significative, les paramètres estimés ne suggèrent pas d'évolution notable vers une plus grande ouverture des sociétés. C'est donc en faveur de la stabilité des régimes de mobilité beaucoup plus que de leur variation prédite par la théorie libérale qu'Erikson et Goldthorpe concluent leur analyse : «Nous disposons bien de résultats probants qui montrent qu'un degré considérable de stabilité des

(4) D'où le nom de «modèle log-multiplicatif de différence uniforme» ou UNIDIFF: qu'Erikson et Goldthorpe attribuent à cette représentation statistique. Le même modèle a aussi été proposé. de manière indépendante ct au même moment, par Xie (1992). Nous le développerons de manière formalisée dans la suite de cet article pour l'utiliser sur les données françaises.

(5) Les auteurs notent cependant qu'un tel résultat n'est pas dans la ligne d'une recherche précédente qui, à partir de la même enquête de 1970 et de données antérieures de 1953, mettait en évidence un affaiblissement de la propension à l'immobilité sociale dans la société française
(Goldthorpe, Portocarero, 1981). Pour expliquer cette divergence, Erikson et Goldthorpe soulignent que cette recherche couvrait une période beaucoup plus longue en incluant des générations masculines plus anciennes. Il est vrai ćgalement que la méthodologie mise en ouvre dans leur ouvrage a pour inconvénient de confondre les effets de gćnération et les effets de cycle de vie (ou de carric̀re) puisqu'elle consiste à comparer les expériences d'hommes qui, tout cn appartenant à des générations distinctes, sont aussi observés à des stades différents de leur vie professionnelle. 
taux relatifs a sous-tendu l'expérience de mobilité des hommes de générations successives, au sein de nos neuf nations; et nous pouvons souligner le contraste entre le caractère plutôt ténu des transformations que nous avons été capables de discerner dans les taux relatifs et les changements beaucoup plus importants et plus rapides dont nous pourrions souvent trouver trace dans les taux absolus, qu'il s'agisse de la mobilité totale ou encore des destinées sociales. » (1992, p. 96). Réunissant les données des neuf pays dans une analyse d'ensemble, Erikson et Goldthorpe montrent enfin que la variation des régimes de mobilité au fil des cohortes a été bien inférieure aux différences de fluidité sociale qui existent entre les sociétés considérées.

Ce n'est pas seulement dans le cadre d'entreprises comparatives, mais aussi au sein d'études nationales que, pour quelques pays au demeurant déjà cités, une tendance progressive à l'égalisation des chances sociales a pu être relevée. Dès 1983, Erikson avait mis en évidence une ouverture graduelle de la société suédoise et ce résultat a été confirmé, plus récemment, à partir de modèles d'analyse plus sophistiqués (Jonsson, Mills, 1993). Une tendance analogue a pu être décrite pour les Pays-Bas entre 1954 et 1977 (Ganzeboom, De Graaf, 1984) comme entre 1970 et 1985 (Luijkx, Ganzeboom, 1989) (6). Elle est aussi solidement documentée, s'agissant de la Hongrie (voir notamment Ganzeboom, Luijkx, Róbert, 1989; Wong, Hauser, 1992). Le cas des États-Unis mérite enfin une attention particulière. Trois années après que Hauser a formulé l'hypothèse d'invariance temporelle du régime de mobilité sociale entre générations, sa «présomption initiale» a dû être «substantiellement modifiée » (Featherman, Hauser, 1978, p. 137) à partir de la comparaison, à onze ans de distance, des données recueillies par Blau et Duncan avec celles de l'enquête Occupational changes in a generation de 1973. Dans les résultats de leurs analyses, Featherman et Hauser observent en effet un ensemble de changements qui les incite à conclure en faveur d'un accroissement des chances relatives de mobilité sociale entre générations (7). Ultérieurement, Hout (1984) a confirmé qu'audelà des transformations structurelles de la société américaine, l'association statistique entre statut du père et statut du fils - mesurés au moyen de l'indice socio-économique de Duncan - avait décru en moyenne de $28 \%$ entre 1962 et 1973. Le même auteur a prolongé l'analyse sur une période plus récente à partir des données des General social surveys conduites entre 1972 et 1985 (Hout, 1988). Pour les hommes comme pour les femmes, c'est de nouveau une chute de l'association statistique entre origine et position sociales qui est mise en évidence; elle est d'intensité comparable à celle observée dans la période antérieure. Introduisant le niveau d'éducation obtenu comme variable intermédiaire entre origine et position sociales, Hout $(1984,1988)$ éclaire enfin les ressorts de cette ouverture progressive du régime de mobilité américain. D'une part, pour les individus qui ne disposent pas d'un diplôme universitaire, le lien

(6) Voir aussi, pour une confirmation de ce résultat, Erikson et Goldthorpe, 1992, p. 172.

(7) Cherkaoui (1992, pp. 180-182) a déjà souligné ce point dans sa présentation de l'en- quête conduite par Featherman et Hauser pour reproduire cclle de Blau et Duncan, réalisée onze ans plus tôt. 
entre la position occupée et le milieu d'origine s'affaiblit au cours de la période. D'autre part, la position sociale est indépendante du milieu de naissance parmi les titulaires d'un diplôme d'université dont l'importance relative s'accroît entre 1962 et 1973 comme entre 1972 et 1985. L'augmentation progressive de la fluidité sociale dans la société américaine résulte ainsi pour partie d'un effet de composition : la croissance, dans la population employée, de la part des plus diplômés pour lesquels l'association statistique entre origine et position sociales est la plus faible.

Dans les chapitres qu'ils consacrent à trois sociétés non européennes Australie, États-Unis, Japon -, Erikson et Goldthorpe (1992, pp. 325-326) réexaminent, à l'intérieur de leur nomenclature de classes, les données recueillies par Featherman et Hauser comme celles utilisées par Hout. Reconnaissant qu'une tendance à l'égalisation des chances sociales aurait pu se manifester aux Etats-Unis, ils argumentent toutefois, d'un point de vue statistique, qu'elle n'aurait été que légère. Par exemple, lorsqu'il est appliqué aux cohortes de naissance de l'enquête américaine de 1973, le modèle de différence uniforme dans le régime de mobilité ne s'ajuste pas significativement mieux aux données que celui de fluidité sociale constante. De même, dans le modèle que Hout utilise, l'affaiblissement du lien entre origine et position sociales qu'il met en évidence ne concerne que la seule dimension du statut alors qu'une tendance inverse paraît se dessiner pour l'autre dimension d'autonomie dans le travail qui, du point de vue d'une analyse de classe, est d'une grande pertinence.

\section{La société française : une quasi-constance de la fluidité sociale?}

Dans notre pays, une proposition largement acceptée parmi les sociologues est que la mobilité sociale a beaucoup augmenté, au cours des dernières décennies, en raison des transformations structurelles qu'a connues la société française, mais que l'inégalité des chances sociales ou la structure de la mobilité y sont en revanche demeurées largement inchangées. Cette dernière conclusion s'est progressivement imposée à partir de la publication, par Raymond Boudon en 1973, d'un ouvrage marquant : L'inégalité des chances. La mobilité sociale dans les sociétés industrielles. Observant que les sociétés occidentales ont connu une diminution de l'inégalité des chances scolaires, mais que la mobilité sociale qui les caractérise ne paraît affectée que de variations faibles et erratiques, l'auteur développe un modèle formel d'allocation des positions sociales en fonction du milicu d'origine et du niveau scolaire et il examine les conséquences qu'engendre, sur un tel système, un processus de démocratisation de l'enseignement (8). Sa conclusion majeure est qu'une diminution de l'inégalité des chances scolaires n'est pas incompatible avec la stabilité de la structure de la mobilité que les données disponibles mettent en évidence. L’inadéquation

(8) Voir en particulier les chapitres 6 (Esquisse d'une théorie formelle de la mobilité sociale) et 7 (Données sur la mobilité sociale et son évolution). 


\section{Revue française de sociologie}

relative de la structure scolaire et de la structure sociale, l'évolution moins rapide de la seconde que de la première, le processus de file d'attente qu'engendre la réduction des espérances sociales attachées aux niveaux scolaires inférieur et moyen, la présence éventuelle d'un effet de dominance qui, à niveau scolaire égal, tend à attribuer les meilleures positions sociales aux individus dont l'origine est la plus élevée, concourent à un tel résultat: «De façon générale, l'augmentation considérable des taux de scolarisation et la démocratisation de l'enseignement n'impliquent ni que la mobilité doive augmenter, ni que sa structure soit modifiée dans le temps. Le modèle conduit à des variations faibles et oscillatoires plutôt qu'unidirectionnelles de la mobilité. Cette conséquence théorique est, comme les précédentes, conforme aux résultats de l'observation. » (Boudon, [1973] 1978, p. 215).

Dans un article récent et sur la base de données françaises obtenues en 1982 et 1994 pour les hommes de 42 à 54 ans qui ont un emploi, Forsé (1997) a réexaminé, d'un point de vue empirique, la théorie développée par Boudon. Sa conclusion est que le modèle en ressort largement confirmé : alors qu'en douze ans et pour des populations comparables, le niveau moyen d'éducation a glissé vers le haut et que l'inégalité des chances scolaires en fonction du milieu d'origine s'est un peu réduite, l'indice de diagonalité nette (Forsé, Chauvel, 1995) des tables de mobilité ne varie pas significativement et l'auteur conclut ainsi, pour la société française, à la «quasi-constance de la fluidité sociale» (p. 234). Un diagnostic identique avait déjà été formulé par Gollac et Laulhé (1987) : ajustant à une table de mobilité de 1977 les distributions socioprofessionnelles de 1985 et la comparant alors aux données récllement obtenues à cette date, ils n'observaient que des variations ténues du régime de mobilité (9). C'est encore la même méthode que Merllié et Prévot (1997, pp. 56-58) mettent en œuvre pour analyser les variations du lien entre origine et position sociales de 1977 à 1985, puis 1993; ils relèvent alors que «les différences, de faible ampleur, sont de sens variable selon les catégories et ne permettent pas de conclure à une évolution significative». Enfin, Goux et Maurin (1997a, 1997b) ont mis à profit les ressources de la modélisation log-linéaire pour analyser les formes du processus d'acquisition du statut, i.e. les liens entre origine sociale, niveau d'études, position à l'entrée dans la vie active et position ultéricure, et leurs variations éventuelles entre 1977 et 1993 (parfois 1970 et 1993). Une de leurs conclusions majeures a trait à la stabilité temporelle de ces relations : « Les inégalités scolaires et sociales ne semblent ni plus ni moins intenses aujourd'hui qu'il y a une ou deux décennies. D'autres choix de nomenclatures (ou de techniques statistiques) auraient peut-être conduit à des estimations et des évaluations un peu différentes. Ces résultats ont toutefois valcur de première approximation. » (1997a, p. 25). Les auteurs parviennent à cette conclusion en

(9) Voir, dans l'article de Gollac et Laulhé, l'encadré «1977-1985: les destinées évoluent peu» (p. 89). I a méthode utiliséc est un algorithme itératif d'ajustement proportionnel (algorithme RAS ou de Deming-Stephan) qui respecte les odds ratios du tableau initial tout en en trans- formant les marges (voir pour une description plus précise Vallet, 1986. p. 673). Cette procédure est en fait équivalente à l'estimation d'un modèle log-linéaire sans interaction entre origine, position et temps, modèle qui exprime l'hypothèse de constance de la fluidité sociale. 
s'appuyant sur un double constat : d'une part, quel que soit l'aspect étudié dans le processus d'acquisition du statut, un modèle statistique qui postule que les relations fondamentales (10) ne se transforment pas au cours du temps reconstruit correctement les données observées dans les enquêtes françaises; d'autre part, lorsqu'une telle interaction avec le temps est introduite, elle ne s'avère pas significative d'un point de vue statistique dans un test global (11).

Les recherches les plus récentes concluent donc en faveur de la stabilité de l'inégalité des chances sociales dans la France des dernières décennies. On argumentera ici que, pour deux ordres de raisons, cette conclusion mérite d'être interrogée et de faire l'objet d'un examen plus approfondi. Le premier aspect est méthodologique. On conviendra aisément que le régime de mobilité d'une socićté en forme une caractéristique structurelle qui, à supposer qu'elle se transforme, ne le fait que lentement, au fil du temps et des générations. Cette évolution - hypothétique - est donc, par nature, difficile à mettre en évidence. Aussi exige-t-elle d'être étudiée à partir de modèles d'analyse dotés d'une forte puissance statistique, c'est-à-dire qui capturent l'évolution temporelle éventuelle en un nombre restreint de paramètres. Tel est précisément le sens de la remarque - citée plus haut - d'Erikson et Goldthorpe (1992, pp. 90-91) comme de leur introduction, au-delà du modèle de fluidité sociale constante, du modèle de différence uniforme ou encore de celui qui postule une variation dans la propension à l'immobilité. Telle est aussi la caractéristique des diverses recherches étrangères présentées dans la première partic. Or, les travaux qui viennent d'être évoqués à propos de la France ont pour point commun de fonder leur conclusion sur la scule prise en considération du modèle d'invariance temporelle ou d'un équivalent de celui-ci (12). Que la réponse apportée apparaisse en faveur de la constance pourrait ainsi ne pas devoir être interprété comme le signe qu'il n'existerait aucune variation sur tel ou tel aspect particulier de la fluidité sociale, mais serait aussi susceptible de traduire l'incapacité de la modélisation mise en cuvre à détecter une telle variation - à supposer qu'elle existe.

Une seconde raison invite à remettre sur le métier l'analyse des tendances temporelles de la fluidité sociale, dans la société française: si, comme on l'a souligné, nombre de recherches concluent à la stabilité, ce n'est pas le cas de toutes. Analysant les destinées sociales des hommes de 40 à 59 ans qui occupent un emploi, Claude Thélot (1982) a mis en évidence, pour le troisième quart du siècle, une forte croissance de la mobilité observée. Dans une nomenclature distinguant huit positions sociales, $50,5 \%$ des hommes étaient classés dans la même catégorie sociale que leur père en 1953 ; ils n'étaient plus que $37,8 \%$ en 1977. La majeure partie de cette évolution tenait au fait qu'en vingt-quatre ans

(10) Par exemple, le lien entre origine sociale et niveau d'études atteint, entre niveau d'études atteint et position sociale, ou cncore entre origine et position sociales.

(11) C'est-à-dire un test utilisant la totalité des paramètres relatifs à la liaison entre les trois variables considérées. Par exemple, à supposer que trois dates et six catégories d'origine et de position sociales soient distinguées, un tel test utilise $(3-1)(6-1)(6-1)=50$ degrés de liberté.

(12) La recherche de Forsé représente, à cet égard, une exception; l'indice de diagonalité nette que l'auteur utilise diminue en fait très légèrement - de 0,73 à 0,72 - mais non significativement en douze ans. 
les transformations de la société française avaient accru l'écart entre la structure sociale des pères et celle des fils. Elle ne lui était cependant pas totalement réductible. Mettant en évidence qu'un quart environ de la chute de l'immobilité sociale entre générations provenait d'un affaiblissement de la tendance «pure » à l'hérédité sociale, l'auteur concluait à une certaine ouverture du régime de mobilité : «Dans le cadre de cette analyse on peut dire que la société française s'est légèrement assouplie : d'une génération à la suivante, toutes choses égales d'ailleurs, la tendance à la reproduction s'est atténuée dans la plupart des groupes sociaux. » (1982, p. 79). Cette conclusion est au demeurant confirmée par la recherche de Goldthorpe et Portocarero (1981) et elle est aussi compatible avec les résultats obtenus par Ganzeboom, Luijkx et Treiman (1989) ou encore Wong (1994) à propos de la France. On a enfin pu montrer que, pour la même période, un affaiblissement comparable de la propension à l'immobilité caractérisait aussi l'expérience de mobilité des femmes, par la profession (tableaux père-fille) ou le mariage (tableaux père-mari) (Vallet, 1991, 1992) (13).

Il importe donc d'étudier à quel degré l'ouverture du régime de mobilité de la société française, mise en évidence dans un troisième quart de siècle caractérisé par l'expansion économique que l'on sait, s'est prolongée ou interrompue dans la période ultérieure. Là encore, si la plupart des recherches tendent à conclure à l'arrêt d'un tel mouvement, elles ne sont pas unanimes : utilisant le modèle log-multiplicatif de différence uniforme sur les données françaises de 1970 et 1985, Goldthorpe (1995, pp. 68-69) relève des signes d'un accroissement faible du niveau général de la fluidité sociale sur la période considérée (14).

Aussi la suite de cet article vise-t-elle à réévaluer les tendances temporelles de la mobilité entre générations dans la société française. En comparaison des recherches antérieures, celle-ci se caractérisera par trois éléments nouveaux. En premier lieu, l'examen portera sur une période de quarante ans, du début des années cinquante à la décennie en cours. Dans le champ de la mobilité sociale,

(13) Ce n'est pas pour la seule période des Trente Glorieuses qu unc légère ouverture du régime de mobilité de la société françaisc a pu ĉtre mise au jour. Fukumoto el Grusky (1993) ont réanalysé le corpus de données constitué par l'historien américain William H. Sewell Jr (1985) à partir des registres de mariage de la ville de Marseille en 1821-1822,1846-1851 et 1869. Pour la cité phocéenne, la premic̀re table de mobilité (1821-1822) correspond au crépuscule de la période pré-industrielle; la seconde (1846-1851) intervient après le décollage initial du processus d'industrialisation; la troisième (1869) se situe enfin au point culminant de la « révolution commerciale». Fukumoto et Grusky mettent en évidence une tendance ténue, mais régulière, à l'affaiblissement de la propension à l'immobilité (-10\% durant la premic̀re transition, $-14 \%$ durant la seconde) et à la diminution du niveau général d'association statistique entre origine et position sociales $(-7 \%$ et $-9 \%$ respectivement). Soulignons une dernière fois que de telles évolutions ne peuvent être mises au jour qu'à l'aide d'outils d'analyse d'une fincsse suffisante; comme l'écrivent les auteurs : «Ce que nous devons toutefois noter immédiatement, c'est que le modèle de fluidité sociale constante est un instrument peu sensible pour détecter des changements dans le régime de mobilité sous-jacent. Si nous souhaitons conduire un test plus puissant de l'hypothèse nulle, il nous faut, en premier lieu, imaginer des modèles susceptibles de rendre compte du lien entre origine et position sous une forme parcimonicuse. » (1993, p. 47).

(14) Fixé à 0 pour la table de mobilité de 1970 , le paramètre log-multiplicatif est estimé à -0,06 pour celle de 1985 et celle différence s'avère significative au seuil de un pour cent. 
la France est en effet, avec la Hongrie, l'un des rares pays à disposer, sur une période aussi longue, de données statistiques à la fois fort homogènes, nombreuses, de bonne qualité et assez régulièrement espacées dans le temps. En second lieu, c'est à la fois l'expérience de mobilité des hommes et celle des femmes qui seront étudiées pour parvenir ainsi à une évaluation plus assurée des tendances temporelles. Enfin, notre recherche s'appuiera sur les instruments d'analyse employés aujourd'hui dans ce domaine, notamment le modèle logmultiplicatif de différence uniforme et le modèle log-multiplicatif d'association de Goodman. Nous débuterons en explicitant, à l'aide d'un exemple, la distinction conceptuelle entre mobilité observée et fluidité sociale, puis nous présenterons les données et la nomenclature des positions sociales que nous utilisons. Il sera alors possible de construire des tables de mobilité (destinécs et recrutements) sur une période de quarante ans et d'observer ainsi les transformations des taux absolus de mobilité entre générations. Une procédure simple - fondée sur l'examen de paires de tables de mobilité successives, pour les hommes et les femmes - nous permettra ensuite de mettre en évidence que le niveau général de la fluidité sociale s'est accru continûment en France du début des années cinquante au début des années quatre-vingt-dix. L'existence de cette tendance régulière sera alors confirmée par l'usage de diverses modélisations embrassant, en un seul regard, toute la période considérée. En conclusion, nous discuterons la portée de ces résultats pour la société française et dans le cadre du débat international sur l"évolution des régimes de mobilité des sociétés; nous indiquerons enfin comment cette recherche pourra être poursuivie en vue d'éclairer les ressorts de la tendance de long terme mise en évidence.

\section{Mobilité observée et fluidité sociale versus mobilité structurelle et mobilité nette}

L'analyse de la mobilité sociale entre générations comme l'étude de son évolution temporelle ont longtemps reposé sur la distinction conceptuelle entre mobilité structurelle (ou mobilité «forcée») et mobilité nette (ou «pure», ou encore d'échange ou de circulation). Elle consistait à envisager la mobilité totale, observée dans une société à un instant donné, comme la somme de deux composantes dont l'une - la mobilité structurelle - était vue comme résultant de la seule évolution de la structure sociale au cours du temps (de la génération des pères à celle des fils) et l'autre - la mobilité nette - mesurait le degré de fluidité ou d'ouverture intrinsèque de la société considérée. À partir de cette distinction, une mesure de la mobilité nette était obtenue en défalquant de la quantité de mobilité totale celle due à la seule transformation structurelle ; cette dernière était évaluée à partir de la dissemblance entre la distribution socioprofessionnelle des pères et celle des fils, telle qu'elle apparaît dans les marges de la table de mobilité (voir pour une présentation et une discussion plus approfondies Bertaux, 1969 ; Cuin, 1993, pp. 124-128 et pp. 230-233). 
Posant en réalité plus de problèmes qu'elles n'en résolvent, cette distinction conceptuelle et son opérationalisation sont, depuis une vingtaine d'années, largement tombées en désuétude (15). Elles ont en premier lieu l'inconvénient de définir comme un résidu ce qui intéresse au premier chef le sociologue : la mobilité nette. Plus fondamentalement, on s'est avisé que l'évaluation de la mobilité structurelle était affectée de biais. Divers phénomènes démographiques - nuptialité et fécondité différentielles dans la «génération » des pères, mortalité différentielle dans celle des fils, migrations - ont pour conséquence que la répartition des fils selon le milieu d'origine n'est, en un sens strict, représentative d'aucune structure sociale réelle du passé. Cette observation, que la littérature anglo-saxonne a retenue comme étant la réponse de Duncan (1966) à Kahl, avait en fait été formulée, dès 1950 en France, par Brésard. Enfin, Goldthorpe (1980) a mis l'accent sur la contradiction interne qui existe entre la distinction conceptuelle et sa mise en œuvre : alors que mobilité structurelle et mobilité nette ne peuvent être empiriquement distinguées que par une partition des mouvements individuels observables dans une table, ces concepts n'ont de signification qu'à un niveau supra-individuel ou macrosociologique.

En lui substituant, depuis la fin des années soixante-dix, la distinction entre mobilité observée (ou taux absolus de mobilité) et fluidité sociale (ou taux relatifs de mobilité), les sociologues de la stratification ont retenu l'esprit du paradigme précédent en en laissant de côté les écueils. Abandonnant l'idée contestable qu'il existerait deux types de mobilité, la nouvelle distinction consiste, dans son essence, à reconnaître que les phénomènes de mobilité sociale peuvent - voire doivent - être étudiés selon deux points de vue, différents et complémentaires.

Celui des taux absolus de mobilité - ou de la mobilité observée - consiste à analyser cette dernière telle qu'elle est enserrée et affectée par l'état de la distribution socioprofessionnelle des pères et de celle des fils. Le poids total de l'immobilité, l'examen des destinées comme celui des recrutements sont les outils de cette appréhension en quelque sorte «en surface» qui est aussi la plus proche de l'expérience sensible qu'ont les individus des phénomènes de mobilité.

D'un autre côté, le point de vue de la fluidité sociale - ou des taux relatifs de mobilité - consiste en l'étude de la structure et de la force du lien entre origine et position sociales lorsque cette association statistique est envisagée indépendamment de l'état de la distribution socioprofessionnelle des pères et de celle des fils. Cette appréhension «en profondeur» des tableaux de mobilité utilise une mesure d'association qui dispose de cette propriété d'indépendance à l'égard des distributions marginales - le odds ratio ou rapport des chances relatives - ainsi que la modélisation log-linéaire, méthode statistique permettant de formuler et d'éprouver des hypothèses relatives aux odds ratios (16). Plutôt

(15) Voir cependant, pour une vue d'ensemble de tentatives plus récentes d'opérationalisation de la même distinction, Luijkx (1994).
(16) Pour unc présentation de l'usage de la modćlisation log-linéaire dans l'analyse comparative de données d'enquête, on pourra consulter l'article de Cautrès (1997). 
que de soutenir que la somme d'une part nette et d'une part structurelle constitue la mobilité totale, la nouvelle distinction conceptuelle appréhende donc les phénomènes de mobilité dans les termes suivants : à un instant donné, un régime intrinsèque de mobilité ou de fluidité sociale se combine à l'état des distributions socioprofessionnelles des fils et des pères propre à ce moment historique pour donner naissance à la mobilité observée à cet instant dans la société considérée.

Mobilité observée et fluidité sociale forment ainsi deux approches différentes et complémentaires. En effet, les tables de mobilité obtenues dans une même société à deux dates différentes - ou pour les hommes et les femmes d'une même société, ou encore pour deux sociétés - peuvent différer sous l'angle de la mobilité observée, mais être semblables du point de vue de la fluidité sociale. Dans ce cas, un même «squelette »-le régime de mobilité - est en quelque sorte habillé différemment parce que la structure sociale a évolué au cours du temps - ou que la distribution socioprofessionnelle diffère selon le sexe, ou encore que les sociétés considérées sont parvenues à des niveaux de développement différents et ont connu des histoires distinctes. En d'autres termes, plus statistiques, deux tableaux de contingence peuvent présenter la même association entre variables, mais des marges différentes.

Soit par exemple une société fictive où n'existeraient que deux positions sociales - celle de cadre et celle d'ouvrier - et où la mobilité entre générations a été observée à deux dates entre lesquelles un processus de tertiarisation est intervenu - la part des positions sociales disponibles au niveau cadre a crû de $25 \%$ à $35 \%$.
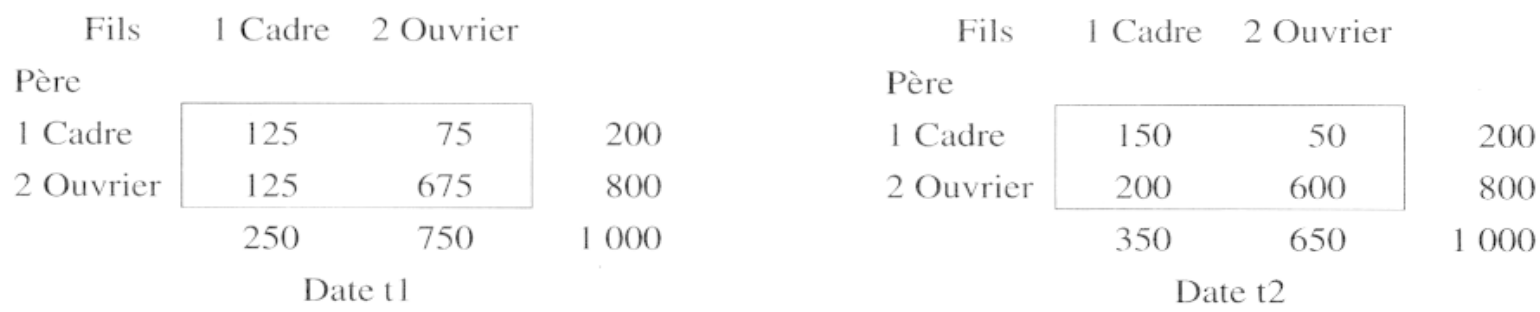

Dans une telle société, la mobilité observée a augmenté au cours du temps. À la date $\mathbf{t} 2$, en effet, un quart des hommes interrogés ne sont pas classés dans la même catégorie sociale que leur père; ils n'étaient que $20 \%$ en $\mathrm{t} 1$. De même, parmi les fils d'ouvrier, les chances d'accès à la position de cadre se sont accrues; de $15,6 \%$ en $\mathrm{t}$, elles passent à $25 \%$ en $\mathrm{t} 2$. Parmi les fils de cadre, la même destinée sociale est aussi devenue plus fréquente : $75 \%$ en $\mathrm{t} 2$ contre $62,5 \%$ précédemment. Toutes ces transformations portent la marque du processus de tertiarisation intervenu qui, en accroissant les chances d'accès à la position de cadre, a aussi augmenté la dissemblance entre la distribution socioprofessionnelle des fils et celle des pères.

Pour autant, l'inégalité relative entre fils de cadre et fils d'ouvrier dans l'accès à la première position plutôt qu'à la seconde a-t-elle varié au cours du temps? Le odds ratio ou rapport des chances relatives qui s'écrit : 


$$
\frac{\left(n_{11} / n_{12}\right)}{\left(n_{21} / n_{22}\right)} \text { ou encore } \frac{\left(n_{11} / n_{21}\right)}{\left(n_{12} / n_{22}\right)} \text {, soit } \frac{\left(n_{11} n_{22}\right)}{\left(n_{12} n_{21}\right)}
$$

traduit, dans sa première expression, le résultat de la concurrence entre les individus des deux origines sociales pour atteindre la première plutôt que la seconde des deux positions (Goldthorpe, 1980). Plus sa valeur est proche de 1, plus cette compétition apparaît égale ou parfaite. Or, il est aisé de calculer pour notre société fictive que cette quantité n'a pas varié de $\mathrm{t} 1$ à $\mathrm{t} 2$ :

$$
\frac{(125 / 75)}{(125 / 675)}=\frac{(150 / 50)}{(200 / 600)}=9
$$

Ainsi, en $\mathrm{t} 2$ comme en $\mathrm{t} 1$, les chances de devenir cadre plutôt qu'ouvrier sont toujours 9 fois plus fortes pour les fils de cadre que pour les fils d'ouvrier. Mesurée par le rapport des chances relatives ou odds ratio, la distance qui sépare les deux milieux d'origine dans l'acquisition de l'une plutôt que de l'autre des deux positions sociales est demeurée inchangée ou encore l'intensité du lien «pur» entre origine et position sociales n'a pas faibli de tl à t2 (17).

Dans la société considérée, la mobilité observée a donc augmenté, mais la fluidité sociale est restée constante. Il n'y a ici nulle contradiction. L'évolution de la mobilité observée dépend en effet à la fois d'une variation éventuelle de la fluidité sociale $e t$ de l'évolution générale de la société que reflètent approximativement les distributions marginales et qui peut être plus ou moins rapide selon le moment historique. Dans notre société fictive, le surcroît de mobilité observée résulte ainsi uniquement de l'écart, croissant de $t 1$ à $t 2$, entre la distribution socioprofessionnelle des fils et celle des pères; il ne traduit en revanche aucune tendance à l'ouverture du régime de mobilité. Savoir si, comme l'ont conclu plusieurs recherches récentes, la société française a connu un développement analogue à celui de notre société fictive forme donc la question centrale de cet article.

\section{Quarante années de mobilité sociale en France : les données disponibles}

Pour étudier l'évolution de la mobilité entre générations sur près d'un demi-siècle, l'enquête réalisée par l'Institut National d'Études Démographiques

(17) Compris entre 0 et $+\infty$, le odds rutio vaut 1 en situation d'indépendance statistique (absence de lien entre origine et position sociales) et l'usage du logarithme néperien rétablit la symétrie de la mesure : I og (odds ratio) est compris entre $-\infty$ et $+\infty$. Pour une démonstration du fait que le odds ratio mesure l'association statistique au cour du tableau de contingence, i.e. indépendamment des distributions marginales, on pourra consulter Vallet (1988, p. 400, note 8). Dans une table de mobilité à NI lignes et NJ colonnes, un ensemble de (NI-1)(NJ-1) odds ratios indépendants suffit à décrire l'association statistique entre origine et position. Enfïn, l'hypothèse d'invariance de la fluidité sociale peut ĉtre représentéc par le modèle log-linéaire d'absence d'interaction entre origine, position et temps : celui-ci suppose que l'association, mesurée par les odds ratios, entre deux variables - origine et position - cst constante selon les modalités de la troisième variable - le temps. 
en 1948 ne peut être mise à profit : elle étudiait la relation entre fécondité et mobilité sociale, mais n'utilisait pas un échantillon visant une représentativité nationale. Il est possible en revanche de faire usage de l'enquête sur l'emploi de juin 1953 : Jacques Desabie $(1954,1955)$ y avait en effet introduit trois questions supplémentaires, dont la suivante : «Lorsque vous avez quitté l'école, quelle était la profession de votre père (ou mère, ou tuteur)? » Les données individuelles de cette enquête ne sont plus disponibles et seuls subsistent des résultats sous forme de tableaux. Ces tables de mobilité sont établies séparément pour les hommes et les femmes, par tranche d'âge décennale. Elles ne permettent pas de distinguer les Français des étrangers et, dans la population active, les individus qui occupent un emploi de ceux qui connaissent le chômage. Nous avons retenu pour l'analyse les hommes et femmes actifs, âgés de 30 à 59 ans au moment de l'enquête ; les tables de mobilité correspondantes portent sur 3483 hommes et 2007 femmes (18).

L'enquête Formation - Qualification Professionnelle conduite par l'Institut National de la Statistique et des Études Économiques en 1964 ne peut, quant à elle, être employée : seuls les individus âgés d'au plus 46 ans y ont déclaré la situation professionnelle de leur père. Nous utilisons en revanche les quatre enquêtes ultérieures de la même série : 1970, 1977, 1985 et 1993. À chaque date, l'indicateur principal d'origine sociale est construit à partir d'une question identique, complétée par d'autres, plus précises : «Quelle était la situation professionnelle de votre père (tuteur) à l'époque où vous avez cessé de fréquenter régulièrement l'école ou l'université ? "Nous avons retenu pour l'analyse l'ensemble des Français, actifs ayant un emploi et âgés de 35 à 59 ans au cours de l'année d'enquête. Il s'agit de 10011 hommes en 1970, 9407 en 1977, 8827 en 1985 et 4013 en 1993 ; pour les femmes, les effectifs correspondants sont respectivement $3561,4556,5178$ et 3182 (19).

Sur une période de quarante ans, nous avons donc à notre disposition cinq tables de mobilité qui rapprochent la position professionnelle d' hommes de celle que détenait leur père à un âge voisin $(\mathrm{N}=35741)$ et nous disposons aussi de tables analogues relatives aux femmes $(N=18484)(20)$. La forte homogénéité de ces données issues d'une même série d'enquêtes nous place dans une situation optimale pour une étude des tendances temporelles. Seule l'enquête de 1953 pourrait être un peu moins comparable aux autres (Thélot, 1983); il conviendra donc de s'assurer que les conclusions obtenues ne reposent pas trop étroitement sur la prise en compte de celle-ci. Le Tableau I dresse une vue synthétique de l'intervalle temporel couvert par notre analyse. Selon leur année de naissance et leur âge de fin d'études, les membres des générations observées dans la table

(18) Ces tables figurent dans Vallet (1991, p. A40).

(19) Ces échantillons représentent environ 5712000 hommes et 3397000 femmes en 1970 , 5971000 et 3700000 en 1977,6139000 et 4404000 en 1985,6937000 et 5399000 en 1993.

(20) Le taux de réponse à l'enquête de 1953 nous est inconnu; il s'élève à $87,8 \%$ en 1970 ,
$86,9 \%$ en $1977,87,8 \%$ en 1985 et $88,3 \%$ en 1993. Pour ces quatre dates, les données que nous analysons sont redressées afin de tenir compte des non-réponses intervenues. Il est done peu probable qu'un biais systématique dans la qualité des enquêtes affecte les analyses que nous conduisons. 
de mobilité la plus ancienne ont quitté le système scolaire et sont entrés sur le marché du travail entre la première décennie du siècle et l'immédiat aprèsSeconde Guerre mondiale. À l'opposé, c'est entre cette dernière période et le début des années quatre-vingt que les générations étudiées dans la table de mobilité la plus récente ont réalisé leur insertion professionnelle. Le même tableau fait apparaître que les trente générations étudiées en 1953 échappent à l'observation et sont entièrement renouvelées dès l'enquête de 1985. De même, la quasi-totalité de celles prises en compte en 1970 sont remplacées lors de l'observation de 1993. À l'évidence, une telle situation est propice à la mise au jour de tendances de long terme - même ténues - qui pourraient exister.

\begin{tabular}{|c|c|c|c|c|c|}
\hline & $\begin{array}{c}1953 \\
(30-59 \text { ans })\end{array}$ & $\begin{array}{c}1970 \\
(35-59 \text { ans })\end{array}$ & $\begin{array}{c}1977 \\
\text { (35-59 ans) }\end{array}$ & $\begin{array}{c}1985 \\
(35-59 \text { ans })\end{array}$ & $\begin{array}{c}1993 \\
(35-59 \text { ans })\end{array}$ \\
\hline $\begin{array}{l}\text { Naissance entre } \\
\hat{A} \text { ge approximatif } \\
\text { de fin d'études entre } \\
\text { Fin des études et entrée } \\
\text { dans la vie active entre }\end{array}$ & $\begin{array}{l}1894 \text { et } 1923 \\
12 \text { et } 25 \text { ans } \\
1906 \text { et } 1948\end{array}$ & $\begin{array}{l}1911 \text { et } 1935 \\
12 \text { et } 25 \text { ans } \\
1923 \text { et } 1960\end{array}$ & $\begin{array}{l}1918 \text { et } 1942 \\
12 \text { et } 25 \text { ans } \\
1930 \text { et } 1967\end{array}$ & $\begin{array}{l}1926 \text { et } 1950 \\
14 \text { et } 25 \text { ans } \\
1940 \text { et } 1975\end{array}$ & $\begin{array}{l}1934 \text { et } 1958 \\
14 \text { et } 25 \text { ans } \\
1948 \text { et } 1983\end{array}$ \\
\hline
\end{tabular}

Lire ainsi

Des 30 générations

observées dans la table

de mobilité de 1953,

13 sont encore présentes

dans celle de 1970 ,

6 dans celle de 1977 ,

aucune en 1985 et 1993.

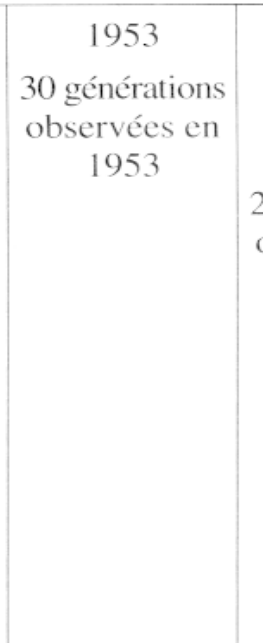

1970

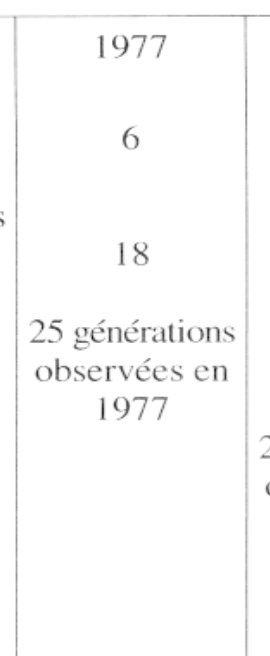

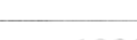

1993

25 générations

observées en 1970

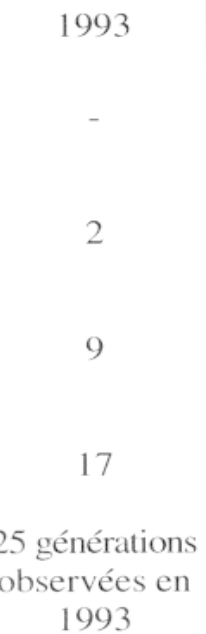

Une difficulté réelle a trait, en revanche, à la nomenclature des positions sociales qui peut être utilisée sur une période aussi longue. I.es tableaux de l'enquête sur l'emploi de 1953 font usage d'un regroupement des Catégories socioprofessionnelles (CSP) qui allaient être employées, dès l'année suivante, pour le recensement de population. Il constitue la nomenclature des positions sociales en huit classes que nous mettons en ceuvre (Tableau II) et qui peut aussi être utilisée sans difficultés pour les enquêtes de 1970 et 1977. Ce regroupement a déjà été employé par Thélot (1982) et il est aussi assez proche de celui effectué par Erikson, Goldthorpe et Portocarero (1979) dans leurs premiers travaux comparatifs sur la France, l'Angleterre et la Suède. Le passage à la nomenclature des Professions et catégories socioprofessionnelles (PCS), intervenu en 1982, rompt en revanche l'homogénéité du codage pour les enquêtes de 1985 et 1993. 
Aussi notre choix a-t-il été d'utiliser la version à deux chiffres de cette nouvelle codification pour reconstruire des catégories aussi proches que possible de celles observées en 1953, 1970 et 1977. La correspondance fournie ci-dessous n'est qu'approximative car la transposition précise des PCS aux CSP (ou inversement) est, en un sens strict, impossible (Seys, 1984). On peut cependant espérer que l'équivalence que nous établissons ne déforme que de façon mineure les faits de mobilité observés et ne nuise ainsi guère à l'étude temporelle de long terme. En outre, les données de l'enquête Formation-Qualification Professionnelle de 1977 ont été recodées a posteriori dans la nomenclature des PCS et cela fournira un moyen de raccorder les séries comme de mieux apprécier les biais éventuels qu'introduisent, dans l'analyse de la longue durée, ces variations des usages statistiques. À l'aide de notre nomenclature des origines et positions sociales en huit classes, il est dès lors possible d'observer en premier lieu l'évolution des taux absolus de mobilité.

TАВLЕАЧ II. - Une nomenclature des origines et positions sociales en huit classes

\begin{tabular}{|c|c|c|}
\hline & Enquêtes de 1953, 1970 et 1977 & Enquêtes de 1977, 1985 et 1993 \\
\hline 1. & Agriculteurs exploitants (CSP OO) & Agriculteurs exploitants (PCS 11, 12, 13) \\
\hline 2. & Salariés agricoles (CSP 10) & Ouvriers agricoles (PCS 69) \\
\hline 3. & $\begin{array}{l}\text { Artisans, petits commerçants, patrons pêcheurs } \\
\text { (CSP 22, 23, 27) }\end{array}$ & $\begin{array}{l}\text { Artisans, commerçants et assimilés } \\
(\text { PCs 21, 22) }\end{array}$ \\
\hline 4. & $\begin{array}{l}\text { Industriels, gros commerçants, professions } \\
\text { libérales (CSP 21, 26, 30) }\end{array}$ & $\begin{array}{l}\text { Chefs d'entreprise de } 10 \text { salariés ou plus, } \\
\text { professions libérales (PCS 23, 31) }\end{array}$ \\
\hline 5. & Cadres supérieurs (Csp 32, 33, 34) & $\begin{array}{l}\text { Cadres et professions intellectuelles supérieures } \\
\text { (PCS } 33 \text { à } 38)\end{array}$ \\
\hline 6. & Cadres moyens (CSp 41 à 44) & $\begin{array}{l}\text { Professions intermédiaires (sauf contremaîtres, } \\
\text { agents de maîtrise) (PCS } 42 \text { à } 47 \text { ) }\end{array}$ \\
\hline 7. & $\begin{array}{l}\text { Employés, personnels de service, autres actifs } \\
(\mathrm{Csp} 51,53,70 \text { à } 72,80 \text { à } 82)\end{array}$ & Employés (PCs 52 à 56) \\
\hline 8. & Contremaîtres et ouvriers (CSP 60 à 68) & $\begin{array}{l}\text { Contremaîtres, agents de maîtrise, ouvriers } \\
\text { (PCS 48, } 62 \text { à 68) }\end{array}$ \\
\hline
\end{tabular}

\section{France 1953-1993 : l’augmentation de la mobilité observée}

Pour la tranche d'âge que nous étudions, 50,7\% des hommes étaient, en 1953 , classés dans la même catégorie sociale que leur père. Cette forte immobilité décroît continûment au cours des décennies suivantes : 40,0\% en 1970, 37,6\% en 1977 (40,2\% à la même date dans la version PCS de notre nomenclature), $36,5 \%$ en $1985,35,1 \%$ enfin en 1993. Rapprocher la position sociale que les femmes occupent par leur emploi de la catégorie paternelle fait apparaître une évolution encore plus prononcée. Pour celles-ci, en effet, la proportion totale d'immobilité s'élève à $47,6 \%$ en $1953,36,2 \%$ en $1970,30,5 \%$ en $1977(30,7 \%$ dans la version PCS), 23,9\% en 1985 et $22,9 \%$ en 1993. 
Ainsi, au milieu du siècle, ce n'était qu'un homme ou une femme sur deux qui, à l'âge de la maturité et du fait de leur situation professionnelle, avaient quitté leur milieu d'origine. C'est le cas d'environ deux hommes sur trois et trois femmes sur quatre au début des années quatre-vingt-dix. Cette forte croissance de la mobilité observée renvoie aux transformations profondes de la France en quarante ans. D'une part, de la transition d'une société comportant un fort secteur agricole à une société industrielle, puis postindustrielle résultent des différences marquées dans la taille des catégories sociales, de la génération des pères à celle des enfants, et, plus ces différences sont fortes, plus il est probable que la mobilité sera élevée (21); d'autre part, au cours de l'industrialisation d'une société, l'importance relative de la paysannerie et de la petite bourgeoisie indépendante décroît et, puisqu'un haut degré d'immobilité entre générations caractérise ces deux classes, il s'ensuit une augmentation de la mobilité observée (Kurz, Müller, 1987).

Le Tableau III et le Tableau IV présentent l'évolution en quarante ans des destinées professionnelles des hommes et femmes originaires des différents milicux sociaux. Les données de l'enquête de 1977 y figurent sous deux formes, qui correspondent aux deux versions de notre nomenclature de classes. En permettant de raccorder les séries, elles donnent aussi à voir les écarts résultant des changements intervenus dans la codification statistique : ils concernent pour l'essentiel la séparation effectuée entre petits et gros indépendants - les postes 3 et 4 de notre nomenclature - ainsi que, de façon plus mineure, le partage entre les employés et les contremaîtres et ouvriers. En dépit de ces variations, l'évolution intervenue en quatre décennies dans les destins sociaux reste le plus souvent nette et bien lisible. On ne relèvera ici que quelques aspects, parmi les plus saillants (22).

Si nombre de transformations sont communes aux hommes et femmes originaires des mêmes milieux sociaux et portent ainsi la marque de la variation générale de la distribution socioprofessionnelle, ce n'est pas le cas de toutes. Au total, le maintien dans la catégorie paternelle est devenu moins fréquent en

(21) La dissemblance entre la distribution socioprofessionnclle des fils (ou filles) et celle des pères peut êtré évaluéc par l'indice de dissimilarité entre les marges du tableau de mobilité. Pour les hommes, il vaut $14.1 \%$ en $1953,21,3 \%$ en $1970,22,4 \%$ en 1977 (22,8\% dans la version PCS de notre nomenclature). $19.1 \%$ en 1985 et $20.9 \%$ en 1993. Pour les femmes, les valeurs correspondantes sont respectivement $22,1 \%$, $27,9 \%, 33,3 \%(39,4 \%$ en version PCS $), 46,5 \%$ et 47,6\%. L'augmentation de la mobilité observée prend donc sa source dans l'écart croissant, mais qui semble plafonner aujourd'hui, entre les structures professionnelles des deux générations. On aura relevé que, pour la proportion totale d'immobilité ou l'indice de dissimilarité entre marges, le passage des CSP aux Prs peut, en 1977 , s'accompagner de variations non négligeables : à cette date, ce sont $18,4 \%$ des hommes et $16,2 \%$ des femmes qui sont classés dans des catégories différentes des deux versions de notre nomenclature en huit postes.

(22) I'utilisation du fichier où figure la recodification $P$ 's de l'enquête de 1977 a conduit, pour le même champ, à retenir un homme de plus (9 408 contre 9407) et deux femmes de plus (4558 contre 4556 ) que dans le fichier original de la même enquête; ces différences sont négligeables. Nous faisons figurer dans les Tableaux III à VI les effectifs totaux non pondérés des lignes ou des colonnes pour donner une indication approchée de la précision des distributions de pourcentages correspondantes. 
quarante ans parmi les hommes originaires de six des huit catégories sociales distinguées; seuls les fils des cadres supérieurs et des cadres moyens font ici exception. Parmi les femmes, il faut ajouter à ces dernières catégories celle des gros indépendants où l'évolution apparaît entachée d'irrégularités et celle des employés. Le classement dans la catégorie paternelle a considérablement chuté chez les enfants des agriculteurs exploitants comme chez ceux des salariés de l'agriculture. Interrompue depuis la fin de la décennie soixante-dix parmi les fils des artisans et commerçants, la diminution de l'immobilité s'est en revanche poursuivie parmi les femmes originaires du même milieu social et cet écart entre sexes tient sans doute à la perte progressive d'importance du statut d'aide familiale dans la population féminine. Bien que plus faible, la diminution de l'immobilité sociale a aussi été régulière jusqu'au milieu des années quatrevingt parmi les fils et filles des contremaîtres et ouvriers; ceux-ci appartiennent plus souvent que par le passé à la catégorie des cadres supérieurs et à celle des cadres moyens (respectivement $11 \%$ et $18 \%$ des fils en 1993 contre $2 \%$ et $8 \%$ en $1953,5 \%$ et $20 \%$ des filles en 1993 contre $0 \%$ et $2 \%$ en 1953 ). On relèvera aussi que, si les femmes originaires de tous les milieux sociaux ont profité de l'expansion considérable des emplois de cadre supérieur - la fréquence de cette destinée sociale croît en effet en quarante ans quelle que soit la catégorie paternelle - il n'en a pas été de même parmi les hommes : dans les années quatre-vingt-dix comme dans les années cinquante, c'est un peu plus de quatre fils de cadre supérieur sur dix qui sont demeurés dans la catégorie paternelle et cette stabilité contraste avec l'augmentation intervenue dans les autres milieux sociaux, notamment chez les fils d'un agriculteur exploitant, d'un artisan ou commerçant, d'un employé, ou encore d'un ouvrier comme on l'a déjà souligné. Enfin, appartenir à «l'archipel des employés » (Chenu, 1990) est un destin social dont la fréquence n'a guère varié en quarante ans parmi les hommes des différentes origines alors qu'elle a crû, souvent de façon importante, chez les femmes qui ont grandi dans la plupart des milieux que notre nomenclature distingue.

Le Tableau V et le Tableau VI adoptent, pour les hommes et les femmes, le point de vue complémentaire du recrutement social des différentes catégories professionnelles. L'autorecrutement des agriculteurs et agricultrices exploitants est resté très stable et très important tout au long de la période étudiée tandis que celui des salariés de l'agriculture a chuté au profit d'une origine plus fréquente dans la catégorie ouvrière. Les artisans et commerçants, hommes et femmes, sont aussi issus moins souvent que par le passé de la petite bourgeoisie indépendante; ils sont en revanche plus nombreux à être enfants d'un contremaître ou d'un ouvrier, mais aussi d'un cadre, supérieur ou moyen. L'évolution du recrutement social de la catégorie des industriels, gros commerçants et professions libérales a suivi des tendances analogues, pour les hommes au moins. L'expansion des professions de cadre supérieur a fait décroître, dans la population masculine, la part des membres de cette catégorie qui en sont eux-mêmes issus, mais le mouvement a été inverse parmi les femmes. Dans les deux cas cependant, le recrutement des cadres supérieurs dans la classe ouvrière est devenu plus fréquent en quarante ans. 
1953 - Hommes français et étrangers, actifs, âgés de 30 à 59 ans 1970, 1977, 1985, 1993 - Hommes français, actifs occupés, âgés de 35 à 59 ans

\begin{tabular}{|c|c|c|c|c|c|c|c|c|c|c|c|}
\hline Fils & & 1 & 2 & 3 & 4 & 5 & 6 & 7 & 8 & Total & $(\mathrm{N})$ \\
\hline \multirow{6}{*}{$\begin{array}{l}1 \text { Agriculteur } \\
\text { exploitant }\end{array}$} & 1953 & 58 & 6 & 8 & 1 & 1 & 3 & 5 & 18 & 100 & 1106 \\
\hline & 1970 & 43 & 5 & 6 & 1 & 2 & 4 & 9 & 30 & 100 & 1973 \\
\hline & 1977 CSP & 38 & 3 & 6 & 2 & 3 & 5 & 10 & 33 & 100 & 2279 \\
\hline & 1977 PCS & 38 & 3 & 8 & 1 & 3 & 6 & 6 & 35 & 100 & 2298 \\
\hline & 1985 & 34 & 2 & 9 & 2 & 4 & 9 & 6 & 34 & 100 & 1771 \\
\hline & 1993 & 27 & 2 & 7 & 1 & 10 & 10 & 8 & 35 & 100 & 772 \\
\hline \multirow{6}{*}{$\begin{array}{l}2 \text { Salarié agricole } \\
\text { (Ouvrier agricole) }\end{array}$} & 1953 & 12 & 29 & 11 & 0 & 0 & 0 & 7 & 41 & 100 & 327 \\
\hline & 1970 & 9 & 16 & 8 & 1 & 2 & 4 & 11 & 49 & 100 & 518 \\
\hline & 1977 CSP & 4 & 12 & 7 & 1 & 2 & 6 & 11 & 57 & 100 & 402 \\
\hline & 1977 PCS & 4 & 12 & 7 & 1 & 2 & 6 & 7 & 61 & 100 & 416 \\
\hline & 1985 & 5 & 7 & 10 & 1 & 3 & 11 & 10 & 53 & 100 & 339 \\
\hline & 1993 & 3 & 5 & 10 & 2 & 4 & 14 & 11 & 51 & 100 & 131 \\
\hline \multirow{6}{*}{$\begin{array}{l}3 \text { Artisan, } \\
\text { petit commerçant } \\
\text { (Artisan, } \\
\text { commerçant et } \\
\text { assimilé) }\end{array}$} & 1953 & 3 & 1 & 47 & 4 & 3 & 6 & 10 & 26 & 100 & 545 \\
\hline & 1970 & 2 & 2 & 26 & 7 & 10 & 12 & 12 & 29 & 100 & 1346 \\
\hline & 1977 CSP & 2 & 1 & 22 & 7 & 13 & 11 & 14 & 30 & 100 & 1192 \\
\hline & 1977 PCS & 2 & 1 & 27 & 4 & 12 & 15 & 8 & 31 & 100 & 1496 \\
\hline & 1985 & 2 & 0 & 26 & 5 & 16 & 17 & 8 & 26 & 100 & 1255 \\
\hline & 1993 & 2 & 0 & 27 & 6 & 17 & 17 & 7 & 24 & 100 & 446 \\
\hline \multirow{6}{*}{$\begin{array}{l}4 \text { Industriel, } \\
\text { gros commerçant, } \\
\text { profession libérale } \\
\text { (Chef d'entreprise } \\
\text { de } 10 \text { salariés ou } \\
\text { plus, profession } \\
\text { libérale) }\end{array}$} & 1953 & 2 & 1 & 12 & 45 & 13 & 6 & 7 & 14 & 100 & 85 \\
\hline & 1970 & 1 & 1 & 11 & 24 & 24 & 13 & 13 & 13 & 100 & 573 \\
\hline & 1977 CSP & 1 & 0 & 14 & 20 & 30 & 12 & 9 & 14 & 100 & 503 \\
\hline & 1977 PCS & 2 & 0 & 7 & 31 & 31 & 14 & 5 & 10 & 100 & 215 \\
\hline & 1985 & 2 & 0 & 15 & 29 & 32 & 13 & 1 & 8 & 100 & 285 \\
\hline & 1993 & 0 & 0 & 9 & 23 & 35 & 18 & 8 & 7 & 100 & 106 \\
\hline
\end{tabular}




\begin{tabular}{|c|c|c|c|c|c|c|c|c|c|c|c|}
\hline \multirow{6}{*}{$\begin{array}{l}5 \text { Cadre supérieur } \\
\text { (Cadre, profession } \\
\text { intellectuelle } \\
\text { supérieure) }\end{array}$} & 1953 & 3 & 0 & 13 & 8 & 43 & 19 & 13 & 1 & 100 & 70 \\
\hline & 1970 & 2 & 0 & 3 & 9 & 45 & 19 & 12 & 10 & 100 & 634 \\
\hline & 1977 CSP & 2 & 0 & 4 & 8 & 45 & 23 & 9 & 9 & 100 & 537 \\
\hline & 1977 PCS & 2 & 0 & 5 & 9 & 45 & 24 & 5 & 10 & 100 & 511 \\
\hline & 1985 & 0 & 0 & 6 & 12 & 46 & 24 & 6 & 6 & 100 & 639 \\
\hline & 1993 & 0 & 0 & 8 & 13 & 41 & 21 & 8 & 9 & 100 & 272 \\
\hline \multirow{6}{*}{$\begin{array}{l}6 \text { Cadre moyen } \\
\text { (Profession } \\
\text { intermédiaire) }\end{array}$} & 1953 & 0 & 0 & 11 & 8 & 13 & 25 & 24 & 19 & 100 & 67 \\
\hline & 1970 & 0 & 0 & 5 & 5 & 27 & 32 & 14 & 17 & 100 & 519 \\
\hline & 1977 CSP & 0 & 0 & 4 & 6 & 30 & 31 & 12 & 17 & 100 & 496 \\
\hline & 1977 PCS & 1 & 0 & 6 & 4 & 27 & 31 & 10 & 21 & 100 & 535 \\
\hline & 1985 & 0 & 0 & 8 & 6 & 31 & 27 & 11 & 17 & 100 & 674 \\
\hline & 1993 & 1 & 0 & 6 & 8 & 31 & 31 & 9 & 14 & 100 & 299 \\
\hline \multirow{6}{*}{$\begin{array}{l}7 \text { Employé, } \\
\text { personnel de } \\
\text { service, } \\
\text { autre actif } \\
\text { (Employé) }\end{array}$} & 1953 & 6 & 0 & 9 & 3 & 11 & 15 & 21 & 35 & 100 & 257 \\
\hline & 1970 & 1 & 1 & 9 & 3 & 12 & 18 & 18 & 38 & 100 & 1241 \\
\hline & 1977 CSP & 1 & 1 & 7 & 2 & 16 & 22 & 18 & 33 & 100 & 1029 \\
\hline & 1977 PCS & 1 & 0 & 9 & 2 & 15 & 24 & 15 & 34 & 100 & 809 \\
\hline & 1985 & 1 & 0 & 9 & 2 & 20 & 24 & 17 & 27 & 100 & 809 \\
\hline & 1993 & 0 & 0 & 7 & 3 & 19 & 26 & 14 & 31 & 100 & 459 \\
\hline \multirow{6}{*}{$\begin{array}{l}8 \text { Contremaître, } \\
\text { ouvrier } \\
\text { (Contremaître, } \\
\text { agent de maîtrise, } \\
\text { ouvrier) }\end{array}$} & 1953 & 3 & 2 & 11 & 0 & 2 & 8 & 12 & 62 & 100 & 1026 \\
\hline & 1970 & 1 & 1 & 7 & 2 & 5 & 12 & 14 & 58 & 100 & 3207 \\
\hline & 1977 CSP & 1 & 1 & 7 & 2 & 7 & 13 & 14 & 55 & 100 & 2969 \\
\hline & 1977 PCS & 1 & 1 & 8 & 1 & 6 & 14 & 10 & 59 & 100 & 3128 \\
\hline & 1985 & 1 & 0 & 10 & 1 & 10 & 16 & 11 & 51 & 100 & 3055 \\
\hline & 1993 & 0 & 0 & 8 & 1 & 11 & 18 & 11 & 51 & 100 & 1528 \\
\hline \multirow[t]{6}{*}{ Ensemble } & 1953 & 22 & 6 & 15 & 3 & 3 & 6 & 10 & 35 & 100 & 3483 \\
\hline & 1970 & 14 & 3 & 9 & 4 & 8 & 11 & 13 & 38 & 100 & 10011 \\
\hline & 1977 CSP & 11 & 2 & 9 & 4 & 11 & 12 & 13 & 38 & 100 & 9407 \\
\hline & 1977 PCS & 11 & 2 & 11 & 2 & 10 & 14 & 9 & 41 & 100 & 9408 \\
\hline & 1985 & 8 & 1 & 12 & 4 & 14 & 16 & 9 & 36 & 100 & 8827 \\
\hline & 1993 & 5 & 1 & 10 & 4 & 16 & 19 & 10 & 35 & 100 & 4013 \\
\hline
\end{tabular}


1953 - Femmes françaises et étrangères, actives, âgées de 30 à 59 ans

\begin{tabular}{|c|c|c|c|c|c|c|c|c|c|c|c|}
\hline Fille & & 1 & 2 & 3 & 4 & 5 & 6 & 7 & 8 & Total & $(\mathrm{N})$ \\
\hline \multirow{6}{*}{$\begin{array}{l}1 \text { Agriculteur } \\
\text { exploitant }\end{array}$} & 1953 & 65 & 2 & 12 & 0 & 0 & 2 & 10 & 9 & 100 & 781 \\
\hline & 1970 & 52 & 1 & 10 & 0 & 1 & 4 & 15 & 17 & 100 & 865 \\
\hline & 1977 CSP & 43 & 1 & 8 & 1 & 1 & 6 & 22 & 18 & 100 & 1065 \\
\hline & 1977 PCS & 43 & 1 & 8 & 0 & 1 & 6 & 28 & 13 & 100 & 1072 \\
\hline & 1985 & 32 & 1 & 9 & 0 & 2 & 11 & 34 & 11 & 100 & 1042 \\
\hline & 1993 & 21 & 0 & 8 & 0 & 3 & 17 & 40 & 11 & 100 & 634 \\
\hline \multirow{6}{*}{$\begin{array}{l}2 \text { Salarié agricole } \\
\text { (Ouvrier agricole) }\end{array}$} & 1953 & 19 & 13 & 20 & 0 & 0 & 2 & 24 & 22 & 100 & 160 \\
\hline & 1970 & 12 & 9 & 11 & 0 & 0 & 3 & 36 & 29 & 100 & 164 \\
\hline & 1977 CSP & 13 & 1 & 11 & 0 & 1 & 7 & 39 & 28 & 100 & 211 \\
\hline & 1977 PCS & 13 & 1 & 11 & 0 & 1 & 5 & 49 & 20 & 100 & 208 \\
\hline & 1985 & 8 & 0 & 10 & 1 & 1 & 7 & 56 & 17 & 100 & 217 \\
\hline & 1993 & 5 & 0 & 7 & 1 & 3 & 12 & 47 & 25 & 100 & 124 \\
\hline \multirow{6}{*}{$\begin{array}{l}3 \text { Artisan, } \\
\text { petit commerçant } \\
\text { (Artisan, } \\
\text { commerçant et } \\
\text { assimilé) }\end{array}$} & 1953 & 9 & 0 & 48 & 2 & 3 & 6 & 19 & 13 & 100 & 278 \\
\hline & 1970 & 7 & 0 & 30 & 2 & 2 & 12 & 35 & 12 & 100 & 506 \\
\hline & 1977 CSP & 4 & 0 & 24 & 3 & 5 & 16 & 35 & 13 & 100 & 578 \\
\hline & 1977 PCS & 4 & 0 & 24 & 1 & 5 & 18 & 38 & 10 & 100 & 709 \\
\hline & 1985 & 2 & 0 & 19 & 3 & 8 & 27 & 35 & 6 & 100 & 676 \\
\hline & 1993 & 3 & 0 & 11 & 1 & 13 & 28 & 37 & 7 & 100 & 372 \\
\hline \multirow{6}{*}{$\begin{array}{l}4 \text { Industriel, } \\
\text { gros commerçant, } \\
\text { profession libérale } \\
\text { (Chef d'entreprise } \\
\text { de } 10 \text { salariés ou } \\
\text { plus, profession } \\
\text { libérale) }\end{array}$} & 1953 & 6 & 0 & 12 & 40 & 6 & 12 & 21 & 3 & 100 & 33 \\
\hline & 1970 & 2 & 0 & 26 & 7 & 12 & 26 & 24 & 3 & 100 & 179 \\
\hline & 1977 CSP & 2 & 0 & 16 & 7 & 13 & 26 & 30 & 6 & 100 & 228 \\
\hline & 1977 PCS & 0 & 0 & 8 & 13 & 16 & 32 & 28 & 3 & 100 & 103 \\
\hline & 1985 & 5 & 0 & 11 & 9 & 21 & 28 & 24 & 2 & 100 & 169 \\
\hline & 1993 & 2 & 0 & 4 & 17 & 24 & 25 & 26 & 2 & 100 & 79 \\
\hline
\end{tabular}




\begin{tabular}{|c|c|c|c|c|c|c|c|c|c|c|c|}
\hline \multirow{6}{*}{$\begin{array}{l}5 \text { Cadre supérieur } \\
\text { (Cadre, profession } \\
\text { intellectuelle } \\
\text { supérieure) }\end{array}$} & 1953 & 9 & 0 & 7 & 12 & 14 & 25 & 21 & 12 & 100 & 43 \\
\hline & 1970 & 2 & 0 & 13 & 3 & 15 & 30 & 32 & 5 & 100 & 182 \\
\hline & 1977 CSP & 3 & 0 & 7 & 5 & 24 & 38 & 19 & 4 & 100 & 242 \\
\hline & 1977 PCS & 3 & 0 & 5 & 7 & 19 & 36 & 28 & 2 & 100 & 219 \\
\hline & 1985 & 0 & 0 & 5 & 5 & 27 & 38 & 22 & 3 & 100 & 405 \\
\hline & 1993 & 0 & 0 & 4 & 5 & 33 & 28 & 26 & 4 & 100 & 245 \\
\hline \multirow{6}{*}{$\begin{array}{l}6 \text { Cadre moyen } \\
\text { (Profession } \\
\text { intermédiaire) }\end{array}$} & 1953 & 0 & 0 & 13 & 3 & 8 & 30 & 41 & 5 & 100 & 37 \\
\hline & 1970 & 2 & 0 & 7 & 3 & 12 & 35 & 30 & 11 & 100 & 196 \\
\hline & 1977 CSP & 0 & 0 & 13 & 5 & 9 & 32 & 35 & 6 & 100 & 272 \\
\hline & 1977 PCS & 1 & 0 & 16 & 2 & 9 & 35 & 32 & 5 & 100 & 288 \\
\hline & 1985 & 1 & 0 & 6 & 3 & 13 & 35 & 36 & 6 & 100 & 429 \\
\hline & 1993 & 2 & 0 & 4 & 1 & 19 & 37 & 33 & 4 & 100 & 228 \\
\hline \multirow{6}{*}{$\begin{array}{l}7 \text { Employé, } \\
\text { personnel de } \\
\text { service, autre actif } \\
\text { (Employé) }\end{array}$} & 1953 & 4 & 0 & 22 & 2 & 0 & 16 & 41 & 15 & 100 & 171 \\
\hline & 1970 & 2 & 0 & 14 & 2 & 3 & 18 & 43 & 18 & 100 & 452 \\
\hline & 1977 CSP & 2 & 0 & 13 & 1 & 6 & 22 & 41 & 15 & 100 & 531 \\
\hline & 1977 PCS & 1 & 0 & 12 & 1 & 4 & 19 & 52 & 11 & 100 & 452 \\
\hline & 1985 & 1 & 0 & 12 & 1 & 7 & 26 & 47 & 6 & 100 & 458 \\
\hline & 1993 & 1 & 0 & 4 & 1 & 13 & 26 & 46 & 9 & 100 & 359 \\
\hline \multirow{6}{*}{$\begin{array}{l}8 \text { Contremaître, } \\
\text { ouvrier } \\
\text { (Contremaître, } \\
\text { agent de maîtrise, } \\
\text { ouvrier) }\end{array}$} & 1953 & 8 & 2 & 17 & 1 & 0 & 2 & 32 & 38 & 100 & 504 \\
\hline & 1970 & 3 & 0 & 11 & 1 & 1 & 9 & 43 & 32 & 100 & 1017 \\
\hline & 1977 CSP & 4 & 0 & 9 & 1 & 3 & 14 & 42 & 27 & 100 & 1429 \\
\hline & 1977 PCS & 3 & 0 & 10 & 0 & 2 & 14 & 47 & 24 & 100 & 1507 \\
\hline & 1985 & 2 & 0 & 9 & 1 & 3 & 19 & 50 & 16 & 100 & 1782 \\
\hline & 1993 & 1 & 1 & 5 & 0 & 5 & 20 & 50 & 18 & 100 & 1141 \\
\hline \multirow[t]{6}{*}{ Ensemble } & 1953 & 31 & 2 & 20 & 2 & 1 & 5 & 21 & 18 & 100 & 2007 \\
\hline & 1970 & 19 & 1 & 14 & 1 & 3 & 11 & 31 & 20 & 100 & 3561 \\
\hline & 1977 CSP & 14 & 0 & 12 & 2 & 4 & 15 & 34 & 19 & 100 & 4556 \\
\hline & 1977 PCS & 15 & 0 & 12 & 1 & 3 & 15 & 39 & 15 & 100 & 4558 \\
\hline & 1985 & 9 & 0 & 10 & 2 & 6 & 21 & 41 & 11 & 100 & 5178 \\
\hline & 1993 & 5 & 1 & 6 & 1 & 10 & 23 & 42 & 12 & 100 & 3182 \\
\hline
\end{tabular}


953 - Hommes français et étrangers, actifs, âgés de 30 à 59 ans

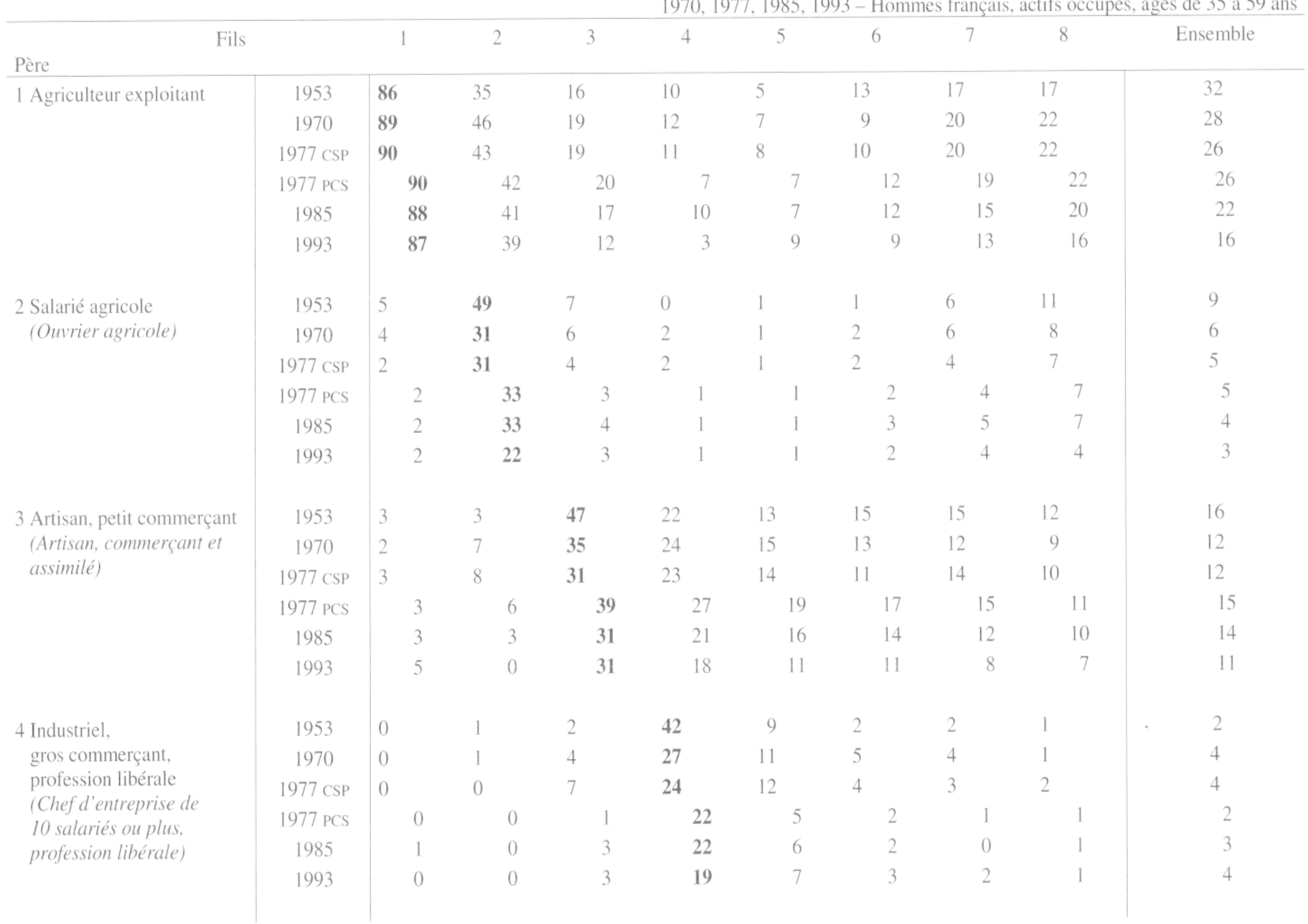




\begin{tabular}{|c|c|c|c|c|c|c|c|c|c|c|}
\hline \multirow{6}{*}{$\begin{array}{l}5 \text { Cadre supérieur } \\
\text { (Cadre, profession } \\
\text { intellectuelle supérieure) }\end{array}$} & 1953 & 0 & 0 & 2 & 7 & 25 & 6 & 3 & 0 & 2 \\
\hline & 1970 & 1 & 0 & 1 & 9 & 20 & 7 & 3 & 1 & 4 \\
\hline & 1977 CSP & 1 & 0 & 2 & 10 & 18 & 9 & 3 & 1 & 5 \\
\hline & 1977 PCS & 1 & 0 & 2 & 15 & 20 & 7 & 2 & 1 & 4 \\
\hline & 1985 & 0 & 2 & 3 & 19 & 19 & 9 & 4 & 1 & 6 \\
\hline & 1993 & 0 & 0 & 6 & 23 & 18 & 8 & 6 & 2 & 7 \\
\hline \multirow{6}{*}{$\begin{array}{l}6 \text { Cadre moyen } \\
\text { (Profession intermédiaire) }\end{array}$} & 1953 & 0 & 0 & 1 & 5 & 8 & 8 & 5 & 1 & 2 \\
\hline & 1970 & 0 & 0 & 2 & 5 & 12 & 11 & 4 & 2 & 4 \\
\hline & 1977 CSP & 0 & 1 & 2 & 7 & 12 & 11 & 4 & 2 & 4 \\
\hline & 1977 PCS & 0 & 0 & 3 & 8 & 13 & 11 & 6 & 2 & 5 \\
\hline & 1985 & 0 & 0 & 4 & 12 & 14 & 11 & 7 & 3 & 6 \\
\hline & 1993 & 1 & 4 & 5 & 15 & 15 & 13 & 7 & 3 & 8 \\
\hline \multirow{6}{*}{$\begin{array}{l}7 \text { Employé, personnel de } \\
\text { service, autre actif } \\
\text { (Employé) }\end{array}$} & 1953 & 2 & 0 & 4 & 9 & 24 & 18 & 15 & 7 & 7 \\
\hline & 1970 & 1 & 3 & 10 & 8 & 16 & 18 & 16 & 11 & 11 \\
\hline & 1977 CSP & 1 & 3 & 9 & 7 & 15 & 19 & 15 & 9 & 11 \\
\hline & 1977 PCS & 1 & 1 & 7 & 7 & 13 & 14 & 14 & 7 & 8 \\
\hline & 1985 & 1 & 4 & 7 & 4 & 12 & 13 & 17 & 7 & 9 \\
\hline & 1993 & 1 & 7 & 9 & 7 & 14 & 17 & 17 & 11 & 12 \\
\hline \multirow{6}{*}{$\begin{array}{l}8 \text { Contremaître, ouvrier } \\
\text { (Contremaître, agent de } \\
\text { maîtrise, ouvrier) }\end{array}$} & 1953 & 4 & 12 & 21 & 5 & 15 & 37 & 37 & 51 & 30 \\
\hline & 1970 & 3 & 12 & 23 & 13 & 18 & 35 & 35 & 46 & 31 \\
\hline & 1977 CSP & 3 & 14 & 26 & 16 & 20 & 34 & 37 & 47 & 33 \\
\hline & 1977 PCS & 3 & 18 & 25 & 13 & 22 & 35 & 39 & 49 & 35 \\
\hline & 1985 & 5 & 17 & 31 & 11 & 25 & 36 & 40 & 51 & 36 \\
\hline & 1993 & 4 & 28 & 31 & 14 & 25 & 37 & 43 & 56 & 39 \\
\hline Total (pour chaque date) & & 100 & 100 & 100 & 100 & 100 & 100 & 100 & 100 & 100 \\
\hline \multirow[t]{6}{*}{$(\mathrm{N})$} & 1953 & 756 & 192 & 538 & 91 & 119 & 215 & 342 & 1230 & 3483 \\
\hline & 1970 & 565 & 176 & 798 & 581 & 1688 & 1535 & 985 & 3683 & 10011 \\
\hline & 1977 CSP & 941 & 156 & 753 & 473 & 1463 & 1343 & 1034 & 3244 & 9407 \\
\hline & 1977 PCS & 950 & 148 & 993 & 341 & 1280 & 1477 & 703 & 3516 & 9408 \\
\hline & 1985 & 649 & 65 & 974 & 425 & 1719 & 1625 & 673 & 2697 & 8827 \\
\hline & 1993 & 333 & 32 & 376 & 159 & 615 & 713 & 391 & 1394 & 4013 \\
\hline
\end{tabular}


1953 - Femmes françaises et étrangères, actives, âgées de 30 à 59 ans 1970, 1977. 1985, 1993 - Femmes françaises, actives occupées, âgées de 35 à 59 ans

\begin{tabular}{|c|c|c|c|c|c|c|c|c|c|c|}
\hline Fille & & 1 & 2 & 3 & 4 & 5 & 6 & 7 & 8 & Ensemb \\
\hline \multirow[t]{6}{*}{1 Agriculteur exploitant } & 1953 & 83 & 28 & 23 & 3 & 14 & 18 & 18 & 18 & 39 \\
\hline & 1970 & 84 & 30 & 22 & 6 & 13 & 11 & 15 & 25 & 30 \\
\hline & 1977 CSP & 81 & 62 & 19 & 11 & 9 & 11 & 18 & 26 & 27 \\
\hline & 1977 PCS & 82 & 70 & 19 & 7 & 7 & 12 & 20 & 23 & 27 \\
\hline & 1985 & 83 & 59 & 20 & 7 & 6 & 13 & 19 & 22 & 23 \\
\hline & 1993 & 74 & 25 & 22 & 0 & 4 & 13 & 17 & 16 & 17 \\
\hline \multirow{6}{*}{$\begin{array}{l}2 \text { Salarié agricole } \\
\text { (Ouvrier agricole) }\end{array}$} & 1953 & 5 & 48 & 8 & 0 & 0 & 3 & 9 & 10 & 8 \\
\hline & 1970 & 4 & 54 & 4 & 2 & 1 & 1 & 7 & 8 & 6 \\
\hline & 1977 CSP & 5 & 8 & 5 & 0 & 1 & 2 & 6 & 8 & 5 \\
\hline & 1977 PCS & 5 & 12 & 5 & 0 & 1 & 2 & 6 & 7 & 5 \\
\hline & 1985 & 4 & 4 & 5 & 2 & 1 & 2 & 6 & 7 & 4 \\
\hline & 1993 & 4 & 0 & 4 & 3 & 1 & 2 & 4 & 7 & 4 \\
\hline \multirow{6}{*}{$\begin{array}{l}3 \text { Artisan, petit commerçant } \\
\text { (Artisan, commerçant et } \\
\text { assimilé) }\end{array}$} & 1953 & 4 & 0 & 34 & 17 & 32 & 16 & 12 & 10 & 14 \\
\hline & 1970 & 5 & 6 & 29 & 19 & 11 & 16 & 15 & 8 & 14 \\
\hline & 1977 CSP & 4 & 8 & 26 & 19 & 15 & 13 & 13 & 9 & 13 \\
\hline & 1977 PCS & 4 & 4 & 30 & 18 & 20 & 19 & 15 & 10 & 15 \\
\hline & 1985 & 3 & 4 & 23 & 26 & 16 & 16 & 11 & 7 & 12 \\
\hline & 1993 & 7 & 12 & 21 & 10 & 15 & 15 & 10 & 7 & 12 \\
\hline \multirow{6}{*}{$\begin{array}{l}4 \text { Industriel, } \\
\text { gros commerçant, } \\
\text { profession libérale } \\
\text { (Chef d'entreprise de } 10 \\
\text { salariés ou plus, } \\
\text { profession libérale) }\end{array}$} & 1953 & 0 & 0 & 1 & 37 & 9 & 4 & 2 & 0 & 2 \\
\hline & 1970 & 0 & 0 & 6 & 22 & 14 & 8 & 2 & 1 & 3 \\
\hline & 1977 CSP & 1 & 0 & 6 & 15 & 11 & 7 & 3 & 1 & 4 \\
\hline & 1977 PCS & 0 & 0 & 1 & 21 & 7 & 3 & 1 & 0 & 2 \\
\hline & 1985 & 1 & 0 & 3 & 14 & 9 & 4 & 1 & 1 & 3 \\
\hline & 1993 & 1 & 0 & 2 & 37 & 6 & 3 & 2 & 0 & 3 \\
\hline
\end{tabular}




\begin{tabular}{|c|c|c|c|c|c|c|c|c|c|c|}
\hline \multirow{6}{*}{$\begin{array}{l}5 \text { Cadre supérieur } \\
\text { (Cadre, profession } \\
\text { intellectuelle supérieure) }\end{array}$} & 1953 & 1 & 0 & 1 & 14 & 27 & 11 & 2 & 1 & 2 \\
\hline & 1970 & 0 & 0 & 3 & 8 & 17 & 9 & 3 & 1 & 3 \\
\hline & 1977 CSP & 1 & 0 & 2 & 12 & 21 & 10 & 2 & 1 & 4 \\
\hline & 1977 PCS & 1 & 0 & 2 & 28 & 20 & 9 & 3 & 0 & 4 \\
\hline & 1985 & 0 & 0 & 3 & 16 & 25 & 10 & 3 & 1 & 6 \\
\hline & 1993 & 0 & 0 & 6 & 30 & 28 & 10 & 5 & 3 & 8 \\
\hline \multirow{6}{*}{$\begin{array}{l}6 \text { Cadre moyen } \\
\text { (Profession intermédiaire) }\end{array}$} & 1953 & 0 & 0 & 1 & 3 & 14 & 11 & 3 & 1 & 2 \\
\hline & 1970 & 1 & 0 & 2 & 11 & 17 & 12 & 3 & 2 & 4 \\
\hline & 1977 CSP & 0 & 0 & 5 & 14 & 10 & 11 & 5 & 1 & 5 \\
\hline & 1977 PCS & 0 & 0 & 6 & 11 & 14 & 12 & 4 & 2 & 5 \\
\hline & 1985 & 1 & 0 & 4 & 14 & 15 & 12 & 6 & 4 & 7 \\
\hline & 1993 & 3 & 0 & 5 & 5 & 14 & 12 & 6 & 3 & 7 \\
\hline \multirow{6}{*}{$\begin{array}{l}7 \text { Employé, } \\
\text { personnel de service, } \\
\text { autre actif } \\
\text { (Employé) }\end{array}$} & 1953 & 1 & 0 & 10 & 12 & 0 & 26 & 16 & 7 & 8 \\
\hline & 1970 & 1 & 1 & 11 & 19 & 15 & 18 & 16 & 10 & 11 \\
\hline & 1977 CsP & 1 & 4 & 13 & 6 & 15 & 16 & 14 & 9 & 11 \\
\hline & 1977 PCS & 1 & 0 & 10 & 7 & 11 & 12 & 12 & 7 & 9 \\
\hline & 1985 & 1 & 0 & 10 & 5 & 9 & 11 & 10 & 5 & 9 \\
\hline & 1993 & 2 & 0 & 8 & 10 & 15 & 13 & 13 & 9 & 12 \\
\hline \multirow{6}{*}{$\begin{array}{l}8 \text { Contremaître, ouvrier } \\
\text { (Contremaître, agent de } \\
\text { maîtrise, ouvrier) }\end{array}$} & 1953 & 6 & 24 & 22 & 14 & 4 & 11 & 38 & 53 & 25 \\
\hline & 1970 & 5 & 9 & 23 & 13 & 12 & 25 & 39 & 45 & 29 \\
\hline & 1977 CSP & 7 & 18 & 24 & 23 & 18 & 30 & 39 & 45 & 31 \\
\hline & 1977 PCS & 7 & 14 & 27 & 8 & 20 & 31 & 39 & 51 & 33 \\
\hline & 1985 & 7 & 33 & 32 & 16 & 19 & 32 & 44 & 53 & 36 \\
\hline & 1993 & 9 & 63 & 32 & 5 & 17 & 32 & 43 & 55 & 37 \\
\hline Total (pour chaque date) & & 100 & 100 & 100 & 100 & 100 & 100 & 100 & 100 & 100 \\
\hline \multirow[t]{6}{*}{$(\mathrm{N})$} & 1953 & 617 & 42 & 395 & 35 & 22 & 103 & 429 & 364 & 2007 \\
\hline & 1970 & 415 & 22 & 453 & 81 & 279 & 658 & 897 & 756 & 3561 \\
\hline & 1977 CSP & 450 & 19 & 379 & 126 & 407 & 864 & 1443 & 868 & 4556 \\
\hline & 1977 PCS & 451 & 18 & 426 & 83 & 310 & 867 & 1685 & 718 & 4558 \\
\hline & 1985 & 333 & 21 & 354 & 136 & 638 & 1283 & 1863 & 550 & 5178 \\
\hline & 1993 & 262 & 9 & 190 & 38 & 292 & 689 & 1306 & 396 & 3182 \\
\hline
\end{tabular}


La composition sociale de la catégorie des cadres moyens et de celle des employés est demeurée assez stable, pour les hommes et les femmes, du milieu à la fin du siècle. Enfin, l'autorecrutement de la classe ouvrière a d'abord diminué jusqu'à la décennie soixante-dix, puis augmente depuis cette date et cette variation, irrégulière, correspond trait pour trait à la croissance, puis à la diminution progressive, de la part des ouvriers et ouvrières fils et filles d'un agriculteur exploitant.

Au total, les changements intervenus dans les taux absolus de mobilité entre générations ont été nombreux en quarante ans au sein de la société française. En particulier, la mobilité observée a beaucoup augmenté. Que la proportion d'individus qui, durant leur existence, demeurent enracinés dans le même milieu ait décliné a pu ainsi donner aux membres de la société le sentiment que les barrières entre catégories sociales s'étaient quelque peu affaissées et que l'inégalité des chances s'était réduite. Savoir s'il en a été ainsi nécessite toutefois d'adopter l'optique de l'analyse de la fluidité sociale ou du régime de mobilité.

\section{La fluidité sociale a augmenté légèrement, mais continûment en France de 1953 à 1993}

Soit deux dates $t$ et $t^{\prime}$, deux origines sociales quelconques $i$ et $i$, deux positions sociales quelconques $j$ et $j$ ' et $o d$ le rapport des chances relatives qui mesure, à la date $t$, la force intrinsèque de l'association statistique entre ces origines et ces positions:

$$
o d=\frac{m_{i j t} m_{i^{\prime} j^{\prime} t}}{m_{i j^{\prime} t} m_{i^{\prime} j t}} \text { ou encore } \log (o d)=\log \left(m_{i j t}\right)+\log \left(m_{i^{\prime} j^{\prime} t}\right)-\log \left(m_{i j^{\prime} t}\right)-\log \left(m_{i^{\prime}{ }^{\prime} t}\right)
$$

L'hypothèse de constance de la fluidité sociale de la date $t$ à la date $t^{\prime}$ peut être exprimée par le modèle log-linéaire d'absence d'interaction entre origine $(\mathrm{O})$, position $(\mathrm{P})$ et temps $(\mathrm{T})$, c'est-à-dire :

$$
\log \left(m_{i j t}\right)=\lambda+\lambda_{i}^{O}+\lambda_{j}^{l^{\prime}}+\lambda_{t}^{T}+\lambda_{i t}^{O T}+\lambda_{j t}^{P T}+\lambda_{i j}^{O P} \quad \text { (Modèle A) }
$$

De l'expression de ce modèle, on déduit en effet :

$$
\log (o d)=\lambda_{i j}^{O P}+\lambda_{i^{\prime} j^{\prime}}^{O P}-\lambda_{i j^{\prime}}^{O P}-\lambda_{i^{\prime} j}^{O P}
$$

i.e. le logarithme de chaque odds ratio s'exprime en fonction de paramètres qui ne sont pas indicés par $t$, ce qui correspond au fait que l'association statistique entre origine et position sociales ne varie pas selon le temps (23).

(23) La présence, au sein du modèle, des paramètres $\lambda$-autres que ceux, $\lambda_{i j}$, relatifs à la liaison entre origine et position - garantit que les tables de mobilité estimées aux dates $t$ et $t$ ' seront dotées des mêmes marges que les tables obser- vées correspondantes. Avec ce type de modèle, c'est donc bien l'association statistique entre origine et position sociales, une fois prise en compte l'évolution des distributions socioprofessionnelles, qui est analysée. 
Considérons désormais le modèle suivant, plus complexe, qui n'est autre que le modèle log-multiplicatif de différence uniforme ou UNIDIFF proposé par Erikson et Goldthorpe (1992) ou encore Xie (1992) :

$$
\log \left(m_{i j t}\right)=\lambda+\lambda_{i}^{O}+\lambda_{j}^{P}+\lambda_{t}^{T}+\lambda_{i t}^{O T}+\lambda_{j t}^{P T}+\beta_{t} \psi_{i j} \quad \text { (Modèle B) }
$$

De l'expression de ce modèle, il est possible de déduire :

$$
\log (o d)=\beta_{t}\left(\psi_{i j}+\psi_{i^{\prime} j^{\prime}}-\psi_{i j^{\prime}}-\psi_{i^{\prime} j}\right)
$$

i.e. le logarithme de chaque odds ratio s'exprime comme le produit de deux ensembles : l'expression formée des paramètres $\psi$ traduit la forme générale indépendante du temps - de l'association statistique entre les origines $i$ et $i$ ' et les positions $j$ et $j$ ' mises en jeu ; le paramètre $\beta_{t}$, spécifique à la date considérée, traduit quant à lui la force relative de l'association entre origine et position sociales à cet instant.

Le modèle UNIDIFF a donc l'intérêt d'exprimer la variation temporelle de la fluidité sociale d'une manière très parcimonieuse : elle prend la forme simple de la comparaison de deux valeurs $\beta_{t}$ et $\beta_{t}{ }^{\prime}$, caractéristiques des dates $t$ ou $t$ ' considérées. Il est d'ailleurs aisé de vérifier que le modèle de fluidité sociale constante ne forme qu'un cas particulier du modèle UNIDIFF obtenu en supposant la stricte égalité des paramètres $\beta_{t}$ et $\beta_{t}$. (fixés alors conventionnellement à 1 ).

Supposons maintenant que, dans l'estimation du modèle UNIDIFF sur les tables de mobilité obtenues aux dates $t$ et $t$ ', la valeur du paramètre $\beta_{t}$ relatif à la première date soit conventionnellement fixée à 1 (24). L'estimation du paramètre $\beta_{t}$. pour la seconde date renseignera alors sur le sens de la variation de la fluidité sociale entre $t$ et $t^{\prime}$. Plus précisément, si $\beta_{t}$, est inférieur à 1 , les logarithmes des odds ratios - qui, à la date $t$ et selon les cas, étaient positifs ou négatifs - seront uniformément plus proches de 0 à la date $t$ '. En d'autres termes, une valeur estimée $\beta_{t}$. inférieure à 1 correspondra au fait que les odds ratios sont uniformément plus proches de 1 , c'est-à-dire que la liaison entre origine et position sociales est moins stricte en $t$ ' qu'elle ne l'était en $t$ ou encore que la fluidité sociale s'est accrue. Inversement, une valeur estimée $\beta_{t}$ ' supérieure à 1 traduira le fait que le lien entre milieu d'origine et position occupée s'est renforcé, c'est-à-dire que le niveau général de la fluidité sociale s'est abaissé ou que l'inégalité des chances sociales a crû au cours de la période (25).

Une telle démarche d'estimation statistique peut être suivie, en premier lieu, en retenant les tables de mobilité masculines obtenues en 1953 et 1970 .

(24) Tel est précisément le cas dans l'algorithme d'estimation que nous utilisons.

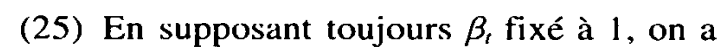
vérifié empiriquement que $\beta_{t}$. valait 0 dans le cas - théorique - où, à la seconde date, la société était caractérisée par l'absence complète de lien entre origine et position sociales, c'est-à-dire par la mobilité ou la fluidité parfaites. En notant $\log (o d)$ ' le logarithme d'un odds ratio quelcon- que, homologue de $o d$, mais relatif à la date $t^{\prime}$, il vient alors :

$$
\begin{aligned}
{\left[\log (o d)^{\prime}-\log (o d)\right] / \log (o d) } & = \\
\left(\beta_{t}-\beta_{t}\right) / \beta_{t} & =\beta_{t} \cdot-\beta_{t}
\end{aligned}
$$

$\beta_{t} \cdot-\beta_{t}$ s'interprète ainsi comme le taux de variation de l'association statistique entre origine et position de $t$ à $t$ '. Par exemple, si $\beta_{r}$. vaut 0,8 , on dira, $\beta_{t}$ valant 1 , que le niveau général de la fluidité sociale s'est accru de $20 \%$ sur la période. 
En l'absence de toute tendance de la fluidité sociale entre ces deux dates au sein de la société française, il existe une chance sur deux que le paramètre $\beta_{1970}$ estimé soit (légèrement) inférieur à 1 et aussi une sur deux qu'il soit (un peu) supérieur à 1 puisque les données, issues d'enquêtes par sondage, sont par nature entachées d'un aléa statistique. Le même exercice peut être reproduit avec les tables masculines de 1970 et 1977 (dans la version CSP de notre nomenclature), avec celles de 1977 et 1985 (en utilisant cette fois la version PCS des données de 1977 afin que la comparaison soit aussi pure que possible), avec celles de 1985 et 1993 enfin. Il est encore possible de procéder de même à partir des tables de mobilité féminines.

Nous disposons ainsi de huit épreuves en vue de répondre à la même question. En l'absence d'une tendance de long terme à l'accroissement de la fluidité sociale dans la société française, la probabilité que tous les paramètres $\beta$ estimés soient inférieurs à 1 est celle d'un événement très rare : $1 /\left(2^{8}\right)$, soit une chance sur 256, ou encore $4 \%$ environ (26). À l'inverse, si un tel résultat était observé dans les valeurs des paramètres $\beta$ estimés, cela constituerait un support empirique fort en faveur de l'idée que l'inégalité des chances sociales s'est réduite continûment dans la société française. Nos estimations sont présentées dans le Tableau VII : le résultat majeur est qu'elles se conforment point par point au schéma général qui vient d'être esquissé.

Fixé à 1 en 1953, le paramètre log-multiplicatif estimé sur les tables de mobilité masculines vaut 0,888 en 1970 et cette différence s'avère significative au seuil de un pour mille (27); ce n'est en revanche pas le cas de la variation, de même sens, mise en évidence sur les tables féminines correspondantes. Entre 1970 et 1977, une tendance à l'accroissement de la fluidité sociale apparaît de nouveau pour les hommes et les femmes; elle est cette fois significative pour les secondes et tout juste non significative pour les premiers au seuil de dix pour cent. S'agissant des périodes 1977-1985 et 1985-1993, le même schéma se reproduit : une tendance à la réduction de l'inégalité des chances sociales est mise au jour et est avérée, au sens d'un test statistique, tantôt pour les hommes, tantôt pour les femmes.

La même analyse peut être reproduite en mettant en jeu des périodes plus longues, fondées sur 1'utilisation des deux versions - CSP, puis PCS - de notre nomenclature des positions sociales. Fixé à 1 en 1953, le paramètre $\beta$ vaut alors 0,900 en 1970 , puis 0,861 en 1977 parmi les hommes (respectivement 0,957 et 0,843 parmi les femmes). Pour les deux sexes, l'amélioration qu'apporte le modèle UNIDIFF par rapport à celui de fluidité sociale constante est en outre significative au seuil de un pour mille. En un quart de siècle, la fluidité sociale

(26) Encore que les épreuves ne soient pas strictement indépendantes puisque ce sont les mêmes dates qui sont mises en jeu pour les hommes et les femmes.

(27) Le logiciel que nous employons ne calcule pas l'erreur-type des paramètres d'un modèle log-multiplicatif. Aussi utilisons-nous, pour les tests statistiques, la différence des khideux du rapport de vraisemblance entre les deux modèles emboîtés, celui de fluidité sociale constante et celui de différence uniforme. 
Louis-André Vallet

TABI.EAU VII. - Estimation du modèle unIDIFF sur les paires de tables de mobilité successives

\begin{tabular}{|c|c|c|c|c|}
\hline Modèle & $\begin{array}{c}\text { Degrés de } \\
\text { liberté }\end{array}$ & Khi-deux & Test & $\begin{array}{c}\text { Paramètres } \\
\text { log-multiplicatifs }\end{array}$ \\
\hline \multicolumn{5}{|l|}{ Hommes } \\
\hline \multicolumn{5}{|l|}{$1953-1970$} \\
\hline A - Fluidité sociale constante & 47 & 108.653 & $\mathrm{p}<.001$ & \\
\hline B - Différence uniforme & 46 & 93.306 & $\mathrm{p}<.001$ & $\beta_{1953}=1.000 \quad \beta_{1970}=0.888$ \\
\hline Différence $(A)-(B)$ & 1 & 15.347 & $\mathrm{p}<.001$ & \\
\hline \multicolumn{5}{|l|}{$1970-1977$} \\
\hline A - Fluidité sociale constante & 49 & 47.231 & ns & \\
\hline B - Différence uniforme & 48 & 44.620 & ns & $\beta_{l 970}=1.000 \quad \beta_{l 977}=0.960$ \\
\hline Différence $(A)-(B)$ & 1 & 2.611 & ns & \\
\hline \multicolumn{5}{|l|}{$1977-1985$} \\
\hline A - Fluidité sociale constante & 47 & 52.163 & ns & \\
\hline B - Différence uniforme & 46 & 47.452 & $\mathrm{~ns}$ & $\beta_{l 977}=1.000 \quad \beta_{l 985}=0.944$ \\
\hline Différence $(\mathrm{A})-(\mathrm{B})$ & 1 & 4.711 & $\mathrm{p}<.05$ & \\
\hline \multicolumn{5}{|l|}{$1985-1993$} \\
\hline A - Fluidité sociale constante & 48 & 71.961 & $\mathrm{p}<.02$ & \\
\hline B - Différence uniforme & 47 & 69.504 & $\mathrm{p}<.02$ & $\beta_{1985}=1.000 \beta_{1993}=0.944$ \\
\hline Différence $(\mathrm{A})-(\mathrm{B})$ & 1 & 2.457 & ns & \\
\hline
\end{tabular}

Femmes $1953-1970$

A - Fluidité sociale constante $\begin{array}{cccc}46 & 87.088 & \mathrm{p}<.001 & \\ 45 & & 85.604 & \mathrm{p}<.001 \quad \beta_{1953}=1.000 \quad \beta_{/ 970}=0.946\end{array}$

B - Différence uniforme

Différence (A) - (B) $1970-1977$

A - Fluidité sociale constante

46

73.619

$$
\begin{aligned}
& \mathrm{p}<.01 \\
& \mathrm{p}<.05 \quad \beta_{1970}=1.000 \quad \beta_{1977}=0.884
\end{aligned}
$$

B - Différence uniforme

Différence (A) - (B)

45

64.834 $1977-1985$

A - Fluidité sociale constante

B - Différence uniforme

Différence (A) - (B)
$45 \quad 58.690 \quad \mathrm{p}<.10$
$44 \quad 58.086 \quad \mathrm{p}<.10 \quad \beta_{1977}=1.000 \quad \beta_{1985}=0.968$
ns

$1985-1993$

A - Fluidité sociale constante

$\begin{array}{lll}45 & 61.441 & \mathrm{p}<.10 \\ 44 & 57.430 & \mathrm{p}<.10\end{array}$

B - Différence uniforme

4.011

$\mathrm{p}<.05$

Différence (A) - (B)

14.011

Hommes français et étrangers, âgés de 30 à 59 ans, actifs ou anciens actifs

(Nomenclature des PCS à un chiffre) 1977 - $1985-1993$

A - Fluidité sociale constante

50

$83.648 \quad \mathrm{p}<.01$

B - Différence uniforme

$48 \quad 62.507$

21.141

$\mathrm{p}<.10$

$2 \quad 21.141 \quad \mathrm{p}<.001$

$\beta_{l 977}=1.000 \beta_{l 985}=0.942$ $\beta_{1993}=0.868$

Le nombre de degrés de liberté de chaque modèle est éventuellement ajusté pour tenir compte de la présence de zéros dans la marge (OP) observée (Bishop, Fienberg, Holland, 1975, pp. 115-119). 
se serait ainsi accrue d'environ $15 \%$ dans la société française. Sur la période 1977-1993 et dans la version PCS de notre nomenclature, une variation de même sens réapparaît encore : fixé à 1 en 1977, le paramètre $\beta$ est, dans le cas des hommes, estimé à 0,945 en 1985 et 0,890 en 1993 ; dans le cas des femmes, les valeurs correspondantes sont 0,965 et 0,870 ; enfin, par rapport au modèle de fluidité sociale constante, l'amélioration de l'ajustement qu'implique la prise en compte de cette tendance par le modèle UNIDIFF est significative au seuil de un pour cent pour les hommes et de cinq pour cent pour les femmes. De nouveau, en seize ans, le niveau général de la fluidité sociale se serait donc accru - ou l'inégalité des chances sociales se serait réduite - d'environ $12 \%$ (28).

Des analyses qui précèdent émerge donc une conclusion qui contraste nettement avec celle obtenue dans les recherches françaises récentes. Loin d'être demeurée inchangée de la fin des années soixante-dix au début de la décennie en cours, la force du lien qui unit le milieu d'origine des individus à la position sociale qu'ils occupent du fait de leur profession aurait au contraire connu une lente érosion et ce mouvement s'inscrirait de surcroît dans le strict prolongement d'une tendance déjà présente, au sein de la société française, depuis le milieu du siècle. Une explication plausible de la divergence entre les conclusions pourrait tenir au fait qu'en utilisant le modèle UNIDIFF, nous disposons en réalité d'un instrument puissant pour détecter des variations, même ténues, du régime de mobilité qui ne pouvaient guère être mises au jour par les recherches précédentes, essentiellement fondées sur le modèle d'invariance temporelle ou sur un équivalent de celui-ci. Avant d'accepter une telle explication, il nous faut cependant reconnaître que notre étude se sépare des précédentes sur un point important, celui de la nomenclature des positions sociales utilisée. Les travaux de Forsé (1997), Goux et Maurin (1997a, 1997b) et Merllié et Prévot (1997) ont en effet en commun d'employer le code des PCS à un chiffre. Or, on ne peut $a$ priori exclure que ce dernier soit plus adapté à la période récente que ne l'est notre nomenclature en huit positions sociales, puisque celle-ci dérive largement du souci d'introduire dans l'analyse les données, désormais anciennes, de 1953. $\mathrm{Si}$ tel était le cas et si les résultats que nous avons obtenus ne pouvaient être reproduits avec l'usage de la nomenclature récente, alors la divergence s'évanouirait et, du même coup, notre conclusion se trouverait invalidée puisqu'elle résulterait de l'usage d'un schéma dont nous pourrions dire qu'il établit, entre les positions sociales, des distinctions aujourd'hui largement périmées.

(28) Rappclons que nos données portent, de 1970 à 1993, sur les hommes et femmes français de 35 à 59 ans qui occupent un emploi. Or, on ne peut a priori exclure que l'abaissement de l'âge légal de fin d’activité et la multiplication, sur la fïn de la période, des formes de retrait anticipé de l'activité professionnelle aient pour conséquence de sélectionner aux âges élevés, en 1985 et 1993 , un ćchantillon peu comparable à celui obtenu en 1977, ce qui pourrait introduire un biais dans les résultats que nous obtenons. Nous avons contrôlé ce point en réitérant nos analyses de la période
1977-1993 de deux manières, i.e. en adoptant un champ plus restreint (hommes et femmes français de 35 à 54 ans qui occupent un emploi) ou plus large (hommes et femmes français de 35 à 59 ans, actifs occupés ou anciens actifs occupés). Les résultats ainsi obtenus - que, par souci de brièveté, nous ne détaillons pas ici - ne diffèrent que très peu de ceux présentés plus haut et ne conduisent donc nullement à infléchir, dans un sens ou dans l'autre, notre conclusion. Ils sont disponibles auprès de l'auteur. 
Afin d'explorer cet aspect, la dernière partie du Tableau VII présente les résultats obtenus sur les tables de mobilité de 1977, 1985 et 1993 construites dans la nomenclature des PCS à un chiffre. La population étudiée est en outre celle retenue par Goux et Maurin dans leur recherche : hommes français et étrangers, âgés de 30 à 59 ans, actifs ou anciens actifs. Des trois études évoquées, c'est en effet celle-ci qui retient le champ de population le plus large. Par rapport à nos analyses antérieures sont donc ajoutés les étrangers, mais aussi les préretraités et les chômeurs ayant déjà travaillé ; seuls manquent les chômeurs n'ayant jamais exercé d'activité professionnelle qui, dans la tranche d'âge considérée, sont en nombre négligeable en 1985 ou 1993. Sur ces données, le modèle de fluidité sociale constante est rejeté au seuil de un pour cent, mais le modèle UNIDIFF ne l'est pas au risque de cinq pour cent et l'amélioration de l'ajustement qu'il apporte s'avère en outre hautement significative. Enfin, les paramètres log-multiplicatifs reproduisent, en l'amplifiant même légèrement, la tendance que nous avons mise au jour : fixé à 1 en $1977, \beta$ vaut en effet 0,942 en 1985 et 0,868 en 1993. Il paraît donc impossible d'accepter la conclusion des auteurs selon laquelle «les inégalités [...] sociales ne semblent ni plus ni moins intenses aujourd'hui qu'il y a une ou deux décennies » (1997a, p. 25) et l'examen attentif des données comme des estimations des modèles fournit des indices éclairants de l'évolution intervenue. Selon l'enquête de 1977 et pour la population retenue, les chances d'être cadre ou profession intellectuelle supérieure plutôt qu'ouvrier étaient 100 fois plus fortes pour les fils de la première origine sociale que pour ceux de la seconde; le même odds ratio vaut 76 dans les données de 1985 et 39 dans celles de 1993. Le modèle de fluidité sociale constante estime à 72 , à chaque date, la valeur de ce rapport des chances relatives, mais le modèle UNIDIFF reproduit en revanche assez fidèlement la tendance présente dans les données d'enquête : les estimations valent 92 en 1977,71 en 1985 et 51 en 1993 (29).

Ainsi, toutes nos analyses convergent et concourent à montrer que, pour les hommes et les femmes, la fluidité sociale s'est accrue légèrement, mais continûment en France, durant quatre décennies. Il est dès lors naturel de tenter d'embrasser, en un seul regard, l'ensemble de la période étudiée. Certes, le prix à payer pour une telle entreprise consiste à mêler, dans la même analyse, trois tables de mobilité - celles de 1953, 1970 et 1977 - qui utilisent la version ancienne de notre nomenclature des positions sociales et deux autres - celles de 1985 et 1993 - qui mettent en jeu la version plus récente. Mais on peut espérer qu'en dépit de l'hétérogénéité introduite la tendance que nous avons mise au jour réapparaisse et cela constituera au demeurant un test de la robustesse de notre résultat. C'est aussi dans le cadre de cette analyse d'ensemble qu'il sera possible d'examiner comment cet accroissement du niveau général de la fluidité

(29) Goux et Maurin portent leur attention sur des tableaux à trois ou plus de trois dimensions, en particulier le tableau croisant origine, diplôme et position sociale; leur modélisation traite donc du lien entre origine et position sociales, à diplôme donné. N'analysant pas le tableau à deux dimensions origine-position qui seul décrit l'association statistique totale entre ces deux variables, ils ne remarquent pas cet indice important d'une ouverture du régime de mobilité entre générations dans la société française. 
sociale peut être réconcilié avec le résultat antérieur, établi pour la période 1953-1977, d'une diminution de la propension à l'immobilité sociale entre générations (Goldthorpe, Portocarero, 1981 ; Thélot, 1982 ; Vallet, 1991, 1992).

\section{Modélisation}

Reprenons les notations introduites précédemment où $t$ désigne une date parmi les cinq considérées, $i$ et $i$ ' sont deux origines sociales quelconques (parmi 8), $j$ et $j$ ' sont deux positions sociales quelconques (parmi 8) et od est le odds ratio qui mesure, à la date $t$, la force du lien entre ces origines et ces positions :

$$
o d=\frac{m_{i j t} m_{i^{\prime} j^{\prime} t}}{m_{i j^{\prime} t} m_{i^{\prime} j t}} \text { ou encore } \log (o d)=\log \left(m_{i j t}\right)+\log \left(m_{i^{\prime} j^{\prime} t}\right)-\log \left(m_{i j^{\prime} t}\right)-\log \left(m_{i^{\prime} j_{t}}\right)
$$

Nous pouvons entamer l'analyse du tableau de contingence croisant origine $(\mathrm{O})$, position $(\mathrm{P})$ et temps $(\mathrm{T})$ à l'aide du modèle log-linéaire suivant :

$$
\log \left(m_{i j t}\right)=\lambda+\lambda_{i}^{O}+\lambda_{j}^{P}+\lambda_{t}^{T}+\lambda_{i t}^{O T}+\lambda_{j t}^{P T}
$$

(Modèle 1)

dont on déduit facilement : $\log (o d)=0$ ou encore $o d=1$. Postulant ainsi que, pour l'année d'enquête considérée, il y a indépendance statistique entre origine et position sociales, un tel modèle exprime l'hypothèse d'une fluidité parfaite à chaque date. Hautement improbable, il fournira néanmoins une base utile pour apprécier l'amélioration de l'ajustement aux données qu'apportent des modèles plus complexes, mais plus réalistes. En ajoutant à l'expression précédente les paramètres $\lambda_{i j}$ relatifs à l'association entre origine et position, nous obtenons le modèle de constance temporelle de la fluidité sociale :

$$
\log \left(m_{i j t}\right)=\lambda+\lambda_{i}^{O}+\lambda_{j}^{P}+\lambda_{t}^{T}+\lambda_{i t}^{O T}+\lambda_{j t}^{P T}+\lambda_{i j}^{O P}
$$

(Modèle 2)

qui implique :

$$
\log (o d)=\left(\lambda_{i j}^{O P}+\lambda_{i^{\prime} j^{\prime}}^{O P}-\lambda_{i j^{\prime}}^{O P}-\lambda_{i^{\prime} j}^{O P}\right)
$$

Nous sommes désormais à pied d'œuvre pour envisager deux directions générales dans l'analyse de la modification temporelle de la fluidité sociale. La première consiste à supposer - en suivant les recherches qui viennent d'être évoquées sur la France des années 1953 à 1977 - que la fluidité sociale est restée constante en dehors d'une variation, au cours du temps, de la propension à l'immobilité entre générations. Une première hypothèse - celle explorée par Erikson et Goldthorpe (1992) - postule que cette variation a été la même dans les différentes classes, mais spécifique à chaque date considérée :

$$
\log \left(m_{i j t}\right)=\lambda+\lambda_{i}^{O}+\lambda_{j}^{P}+\lambda_{t}^{T}+\lambda_{i t}^{o T}+\lambda_{j t}^{P T}+\lambda_{i j}^{O P}+\alpha_{t} d_{i j}
$$
avec $d_{i j}=1$ si $i=j$ et $d_{i j}=0$ sinon d'une part, $\alpha_{1953}=0$ par convention d'autre part (les variations de la propension à l'immobilité seront donc exprimées par comparaison à la première enquête). Un tel modèle implique en effet :

$$
\log (o d)=\left(\lambda_{i j}^{O P}+\lambda_{i^{\prime} j^{\prime}}^{O P}-\lambda_{i j^{\prime}}^{O P}-\lambda_{i^{\prime} j}^{O P}\right) \text { si aucune cellule diagonale n'est mise en jeu, }
$$


alors que, si $i=j$ et $i^{\prime}=j^{\prime}$ :

$$
\begin{aligned}
& \log (o d)=\left(\lambda_{i j}^{O P}+\lambda_{i^{\prime} j^{\prime}}^{O P}-\lambda_{i j^{\prime}}^{O P}-\lambda_{i^{\prime} j}^{O P}\right) \text { en } 1953 \\
& \log (o d)=\left(\lambda_{i j}^{O P}+\lambda_{i^{\prime} j^{\prime}}^{O P}-\lambda_{i j^{\prime}}^{O P}-\lambda_{i^{\prime} j}^{O P}\right)+2 \alpha_{1970} \text { en } 1970, \text { etc. }
\end{aligned}
$$

Une hypothèse plus restrictive que la précédente peut encore être formulée. Elle conduit à un modèle très parcimonieux qui ne diffère de celui d'invariance temporelle que par l'estimation d'un paramètre supplémentaire. Il consiste à supposer que la variation temporelle de la propension à l'immobilité a suivi une tendance linéaire :

$$
\log \left(m_{i j t}\right)=\lambda+\lambda_{i}^{O}+\lambda_{j}^{P}+\lambda_{t}^{T}+\lambda_{i t}^{O T}+\lambda_{j t}^{P T}+\lambda_{i j}^{O P}+\alpha_{l i n} d_{i j t}
$$

avec $d_{i j t}=0$ si $i$ est différent de $j$ alors que, si $i=j, d_{i j t}=0$ en 1953, 17 en 1970, 24 en 1977, 32 en 1985 et 40 en 1993. La différence de khi-deux entre les modèles 2 et 4 - que nous noterons dans la suite (2)-(4) - permettra donc de tester la significativité d'une tendance temporelle linéaire, commune aux différentes classes, dans la variation de la propension à l'immobilité. De même, (4)-(3) éprouvera l'hypothèse que cette tendance linéaire décrit correctement la variation de la propension à l'immobilité entre les différentes enquêtes.

Il est alors possible de desserrer progressivement les contraintes assez fortes du modèle 4, tout d'abord en permettant que la propension à l'immobilité varie, au cours du temps, selon une tendance linéaire spécifique à chaque classe :

$$
\log \left(m_{i j t}\right)=\lambda+\lambda_{i}^{O}+\lambda_{j}^{P}+\lambda_{t}^{T}+\lambda_{i t}^{O T}+\lambda_{j t}^{P T}+\lambda_{i j}^{O P}+\alpha_{i l i n} d_{i j t}
$$

(Modèle 5)

où $d_{i j t}$ est défini comme précédemment; (4)-(5) testera donc le fait que la tendance linéaire dans la variation de l'immobilité sociale n'a pas été la même dans les différentes classes. On peut enfin supposer que la propension à l'immobilité a été spécifique à chaque classe et à chaque date :

$$
\log \left(m_{i j t}\right)=\lambda+\lambda_{i}^{O}+\lambda_{j}^{P}+\lambda_{t}^{T}+\lambda_{i t}^{O T}+\lambda_{j t}^{P T}+\lambda_{i j}^{O P}+a_{i r} d_{i j}
$$

(Modèle 6)

où $d_{i j}$ est défini comme précédemment et les paramètres $\alpha_{i t}$ ont pour effet d'ajuster parfaitement la diagonale de chaque table de mobilité ; (5)-(6) testera donc le fait que les variations temporelles de la propension à l'immobilité dans les différentes classes ne peuvent être résumées par des tendances linéaires.

Nous disposons désormais d'une vision structurée pour la variation temporelle de la propension à l'immobilité sociale entre les différentes enquêtes. Les modèles log-linéaires 3 à 6 ont en commun le point suivant : seuls les odds ratios qui mettent en jeu au moins une cellule diagonale se transforment au cours du temps. À l'opposé, la seconde direction d'analyse - celle d'une évolution du niveau général de la fluidité sociale - implique que tous les odds ratios aient leur valeur qui varie entre les différentes enquêtes. Il faut ici recourir à des modèles plus complexes, de type log-multiplicatif. Cette hypothèse peut alors revêtir deux formes, dont la première - le modèle UNIDIFF - a été utilisée dans la section précédente :

$$
\log \left(m_{i j t}\right)=\lambda+\lambda_{i}^{O}+\lambda_{j}^{P}+\lambda_{t}^{T}+\lambda_{i t}^{O T}+\lambda_{j t}^{P T}+\beta_{t} \psi_{i j}
$$

(Modèle 7) 
qui implique :

$$
\log (o d)=\beta_{t}\left(\psi_{i j}+\psi_{i j^{\prime}}-\psi_{i j^{\prime}}-\psi_{i^{\prime} j}\right)
$$

avec $\beta_{1953}=1$. La seconde forme envisagée dérive des résultats précédents qui semblent mettre au jour une tendance monotone et régulière au sein des paramètres $\beta$. Elle consiste à postuler que ceux-ci ont varié selon une tendance linéaire :

$$
\log \left(m_{i j t}\right)=\lambda+\lambda_{i}^{O}+\lambda_{j}^{P}+\lambda_{t}^{T}+\lambda_{i t}^{O T}+\lambda_{j t}^{P T}+\left(1+\beta_{l i n} \text { year }\right) \psi_{i j}
$$

qui implique :

$$
\log (\text { od })=\left(1+\beta_{l i n} \text { year }\right)\left(\psi_{i j}+\psi_{i^{\prime} j^{\prime}}-\psi_{i j^{\prime}}-\psi_{i^{\prime} j}\right)
$$

où year est une variable quantitative qui vaut 0 en 1953, 17 en 1970, 24 en 1977 , 32 en 1985 et 40 en 1993. Dans un tel cadre, $\beta_{\text {lin }}$ s'interprète comme le taux annuel de variation de l'association statistique entre origine et position sociales - mesurée par le logarithme des odds ratios - sur la période de quarante ans considérée. Comme le modèle 4 , le modèle 8 a l'intérêt d'être extrêmement parcimonieux puisqu'il n'inclut, par comparaison à l'hypothèse de fluidité sociale constante, qu' un paramètre supplémentaire. Enfin, (2)-(8) permettra de tester la significativité d'une tendance linéaire dans la variation temporelle du niveau général de la fluidité sociale tandis que (8)-(7) éprouvera l'hypothèse que cette tendance linéaire traduit correctement les modifications, intervenues entre les différentes enquêtes, de la force du lien entre origine et position.

Une question reste en suspens : à supposer que l'analyse décèle des variations temporelles, à la fois dans la propension à l'immobilité (modèles 3 à 6) et dans le niveau général de la fluidité sociale (modèles 7 et 8), comment savoir si ces deux tendances ont coexisté ou, dans le cas contraire, laquelle a supplanté l'autre et traduit ainsi le mieux la variation présente dans les données d'enquête? Une réponse peut être apportée en réunissant, au sein d'un même modèle, les deux tendances en question. Considérons ainsi le modèle 9 qui combine la tendance linéaire du modèle 4 et celle du modèle 8 :

$$
\log \left(m_{i j t}\right)=\lambda+\lambda_{i}^{O}+\lambda_{j}^{P}+\lambda_{t}^{T}+\lambda_{i t}^{o T}+\lambda_{j r}^{p T}+\alpha_{l i n} d_{i j t}+\left(1+\beta_{l i n} \text { year }\right) \psi_{i j}
$$

(Modèle 9)

(4)-(9) permettra de tester l'hypothèse qu'après prise en compte d'une tendance linéaire dans la propension à l'immobilité sociale entre générations, l'introduction d'une tendance - linéaire également - pour le niveau général de la fluidité sociale s'avère significative; de même, (8)-(9) éprouvera l'hypothèse inverse de la pertinence éventuelle d'une tendance pour l'immobilité après la prise en compte de l'autre. La comparaison des deux résultats fournira ainsi des éléments de décision quant à la réponse à apporter à la question précédente. Il est enfin possible de combiner les caractéristiques du modèle 6 avec celles du modèle 8 :

$$
\log \left(m_{i j t}\right)=\lambda+\lambda_{i}^{o}+\lambda_{j}^{P}+\lambda_{t}^{T}+\lambda_{i t}^{o T}+\lambda_{j t}^{P T}+\alpha_{i t} d_{i j}+\left(1+\beta_{l i n} \text { year }\right) \psi_{i j} \quad \text { (Modèle 10) }
$$

Puisque le modèle 6 ajuste parfaitement l'effectif des cellules diagonales des cinq tables de mobilité et qu'il n'introduit ainsi aucune hypothèse restrictive quant aux variations temporelles dans la propension à l'immobilité, (6)-(10) 
testera donc le fait que, pour les seuls individus mobiles, i.e. ceux qui ont quitté la catégorie paternelle, une variation linéaire du niveau général de la fluidité sociale est intervenue au cours du temps (30).

Nous disposons désormais de dix modèles pour conduire une analyse générale de l'évolution temporelle de la fluidité sociale en France. Après l'estimation de chacun d'eux sur les données d'enquête, le khi-deux du rapport de vraisemblance mesure la distance des effectifs estimés à ceux observés. À partir de cette statistique, il est possible d'évaluer la part du khi-deux du modèle de base celui de fluidité parfaite à chaque date - dont un autre modèle, plus complexe, rend compte. Un second indicateur descriptif de la qualité de l'ajustement obtenu est fourni par l'indice de dissimilarité delta entre le tableau de contingence $5 \times 8 \times 8$ estimé et celui observé : il mesure la proportion de l'effectif total d'individus «qui devraient changer de cellule» pour que le premier tableau devienne identique au second; plus delta est faible, plus le modèle estimé reproduit donc avec fidélité les données observées. Enfin, à partir des valeurs de khi-deux obtenues, il est possible de conduire un test statistique sur le rejet ou le non-rejet d'un modèle comme sur la différence entre deux modèles emboîtés.

La réalisation de ces tests se heurte cependant à une difficulté, désormais commune dans la recherche sociologique quantitative et à laquelle nous n'échappons pas, difficulté liée à la taille souvent très importante des échantillons mis en jeu - plus de 35000 hommes et plus de 18000 femmes dans l'analyse que nous conduisons. Une situation fréquente est en effet que tout modèle ou presque est rejeté, en dehors de celui, saturé, qui décrit parfaitement les données, mais ne les simplifie en rien. Les tests statistiques classiques conduisent ainsi, si on les suit aveuglément, à incorporer de nombreux paramètres dont un certain nombre correspondent à des effets d'amplitude triviale dont l'interprétation peut être malaisée. La même situation se reproduit dans la comparaison entre modèles. Il se pourrait par exemple qu'entre le modèle 4 et le modèle 6 qui, dans notre analyse, diffèrent de 31 degrés de liberté, la différence soit significative alors que la qualité de l'ajustement aux données ne s'améliore en réalité que très peu par l'incorporation de 31 paramètres supplémentaires dont l'examen ne révélerait aucune structure clairement interprétable. Une solution à ce problème délicat de sélection de modèle en présence d'un grand échantillon a été proposée par Raftery $(1986,1995)$ et son usage s'est progressivement diffusé. D'un point de vue pratique, l'indicateur BIC - pour Bayesian Information Criterion - favorise l'adoption d'un modèle relativement parcimonieux en effectuant un arbitrage entre l'écart du modèle aux données et le nombre de paramètres qu'il inclut. Plus précisément, on a pu montrer, dans le contexte de l'approche bayésienne de l'inférence statistique, qu'une valeur

(30) Nous avons en réalité envisagé et testé toutes les combinaisons (au nombre de huit) entre les modèles 3 à 6 et les modèles 7 et 8 . Elles conduisent très largement à un même résultat, pour les hommes comme pour les femmes. Aussi ne retenons-nous ici que les deux combinaisons dont l'interprétation nous paraît être la plus éclairante. 
négative de BIC correspond au fait que le modèle considéré est plus probable que le modèle saturé, étant donné les données; il s'ensuit par ailleurs qu'entre deux modèles emboîtés ou non emboîtés, celui présentant la statistique BIC la plus négative peut être considéré comme le plus vraisemblable et doit donc être préféré (31).

Dans le contexte de notre analyse où la comparaison est à effectuer avec l'hypothèse d'invariance temporelle du régime de mobilité, une règle de décision cohérente peut donc être adoptée : tout modèle qui, par rapport à celui de fluidité sociale constante, ajoute une (des) tendance(s) statistiquement significative(s), mais fournit une statistique BIC moins négative, ne devra pas être préféré à ce dernier; ce sera en revanche le cas chaque fois que la tendance introduite s'avérera significative et que le BIC obtenu sera plus négatif que celui associé à l'hypothèse d'invariance temporelle. On considérera alors que la tendance en question est d'importance suffisante pour être prise en compte et interprétée d'un point de vue théorique.

\section{La fluidité sociale a augmenté au rythme de $0,5 \%$ par an durant quarante ans}

Réalisée sur les tables de mobilité masculines, l'analyse générale fournit les résultats présentés dans le Tableau VIII. Bien qu'il soit rejeté au terme d'un test statistique classique, le modèle de constance de la fluidité sociale ne classe de manière erronée que moins de $3 \%$ de l'effectif total d'hommes considéré et il élimine près de $98 \%$ de la distance des données au modèle de base - celui de fluidité parfaite à chaque date. Fournissant de surcroît une statistique BIC très négative (-1654), le modèle d'invariance apparaît ainsi comme un candidat très sérieux à la description de la fluidité sociale - ou de l'inégalité des chances sociales - en France entre 1953 et 1993.

Cependant, en envisageant les modèles qui permettent que la propension à l'immobilité entre générations se modifie au cours du temps, nous observons, à partir des paramètres estimés sous le modèle 3 , une diminution régulière de celle-ci et cette diminution peut en outre être résumée, sans perte d'information notable, par un trend linéaire. Le modèle 4 révèle ainsi une tendance générale, très significative, à l'affaiblissement de la propension à l'immobilité et, puisqu'il fournit une statistique BIC nettement inférieure à celle obtenue sous le modèle d'invariance (-1670), doit être préféré à ce dernier. Au-delà de ce modèle, les variations - linéaires, puis non linéaires - de la propension à

(31) Bic se calcule au moyen de la formule : $k h i$-deux - (ddl) $\log N$, où $d d l$ est le nombre de degrés de liberté du modèle et $N$ désigne la taille de l'échantillon. Dans le Iong chapitre de Sociological methodology 1995 (p. 139, tableau 6) qu'il consacre à l'approche bayésienne de la sé- lection de modèle, Raftery précisc qu'entre deux modèles, une différence de BIC comprise entre 6 et 10 points correspond à une évidence forte (et une différence supérieure à 10 points à une évidence très forte) en faveur du modèle au BIC le plus faible. 
l'immobilité entre les différentes classes sont elles aussi significatives, mais, la valeur de BIC associée à ces hypothèses se détériorant, c'est encore, des modèles 3 à 6 , le modèle 4 qui doit être préféré.

S'agissant de la variation du niveau général de la fluidité sociale, le modèle UNIDIFF (modèle 7) met au jour une diminution régulière de la force du lien entre origine et position sociales. Fixé à 1 en 1953, le paramètre $\beta$ décline en effet jusqu'à valoir 0,806 en 1993 et indique ainsi une augmentation de la fluidité sociale de l'ordre de $20 \%$ en quatre décennies. Et, de nouveau, cette modification peut être résumée, avec une perte d'information négligeable, par un trend linéaire très significatif. En raison de la statistique BIC qu'il procure $(-1679)$, le modèle UNIDIFF muni d'une tendance linéaire doit ainsi être préféré à celui d'invariance. Il conduit à conclure, $\beta_{l i n}$ valant $-0,0046$, que la fluidité sociale s'est accrue au rythme annuel de $0,5 \%$ durant toute la période étudiée.

Enfin, réunir au sein du modèle 9 les deux tendances mises au jour conduit, une fois encore, à une description de l'évolution qui, selon la statistique BIC, doit être préférée à l'hypothèse d'invariance temporelle et réalise de plus un arbitrage entre les deux trends mis en jeu. En effet, l'introduction d'une tendance linéaire dans le niveau général de la fluidité sociale après celle d'une tendance analogue pour la propension à l'immobilité s'accompagne d'une amélioration significative de l'ajustement (différence entre les modèles 4 et 9), mais l'inverse n'est pas vrai (différence entre les modèles 8 et 9). Qu'une modification générale de la fluidité sociale existe ainsi au-delà de la variation dans la propension à l'immobilité est encore confirmé par le modèle 10 : cette tendance, estimée sur les seuls individus qui ont quitté la catégorie paternelle, est significative au seuil de un pour mille et, $\beta_{\text {lin }}$ valant $-0,0060$, n'est en aucun cas plus faible que celle décelée sur l'ensemble du tableau de mobilité. Au total et en raison de ces résultats, c'est le modèle UNIDIFF muni d'une tendance linéaire (modèle 8 ) qui décrit le mieux l'évolution intervenue; de tous les modèles considérés, c'est du reste celui dont la statistique $B I C$ est la plus négative (32).

La même analyse générale, réalisée sur les tables de mobilité féminines, conduit aux résultats du Tableau IX. Il ne nécessite pas un commentaire approfondi car un examen attentif suffit à se convaincre que les conclusions relatives à la mobilité des femmes ne diffèrent en rien de celles établies pour les hommes. Ce sont de nouveau trois modèles - les mêmes que précédemment qui, du fait de leur statistique BIC, doivent être préférés à celui d'invariance temporelle. C'est encore le modèle UNIDIFF muni d'une tendance linéaire (modèle 8) qui, au terme d'un examen analogue à celui détaillé dans le cas

(32) John H. Goldthorpe a attiré notre attention sur le fait que la validité de l'indicateur BIC n'était pas unanimement acceptée (voir notamment Weakliem, 1992, pp. 171-173). Nous voudrions préciser que le cadre dans lequel BIC a été proposé nous semble bien correspondre à notre recherche, i.e. une situation où le problème n'est pas tant de sélectionner le vrai modèle que de retenir celui qui fournit le plus d'information par rapport au monde réel (Hagenaars, 1990, pp. 6668 ; Vermunt, 1997, p. 22). Par ailleurs, un autre indicateur d'usage fréquent - le critère d'information d'Akaike - conduit aussi, pour les hommes et les femmes, à préférer le modèle 8 au modèle de fluidité sociale constante comme aux modèles 3,4 et 9 . 
TABLEAL VIII. - Analyse de la variation temporelle de la fluidité sociale en France, 1953-1993 Hommes $(N=35741)$

\begin{tabular}{|c|c|c|c|c|c|c|}
\hline Modèle & $\begin{array}{l}\text { Degrés de } \\
\text { liberté }\end{array}$ & Khi-deux & Test & $\operatorname{Delta}(\%)$ & $\begin{array}{l}\text { Part de khi-deux } \\
\text { expliquée }(\%)\end{array}$ & BIC \\
\hline 1 - Fluidité parfaite à chaque date & 245 & 17340.758 & $\mathrm{p}<.001$ & 25.6 & - & 14772.2 \\
\hline 2 - Fluidité sociale constante & 196 & 401.258 & $\mathrm{p}<.001$ & 2.8 & 97.7 & -1653.6 \\
\hline \multicolumn{7}{|l|}{ Variation de la propension à l'immobilité } \\
\hline $\begin{array}{c}\text { 3- Variation commune aux classes } \\
\text { et spécifique à l'année }\end{array}$ & 192 & 368.240 & $\mathrm{p}<.001$ & 2.6 & 97.9 & -1644.7 \\
\hline 4 - Tendance linéaire commune aux classes & 195 & 374.079 & $\mathrm{p}<.001$ & 2.7 & 97.8 & -1670.3 \\
\hline Différence (2) - (4) & 1 & 27.179 & $\mathrm{p}<.001$ & & & \\
\hline Différence (4) - (3) & 3 & 5.839 & ns & & & \\
\hline 5 - Tendance linéaire spécifique à chaque classe & 188 & 336.411 & $\mathrm{p}<.001$ & 2.5 & 98.1 & -1634.6 \\
\hline Différence (4) - (5) & 7 & 37.668 & $\mathrm{p}<.001$ & & & \\
\hline $\begin{array}{l}\text { 6- Variation spécifique à chaque classe } \\
\text { et à chaque date }\end{array}$ & 164 & 270.364 & $\mathrm{p}<.001$ & 1.9 & 98.4 & -1449.0 \\
\hline Différence $(5)-(6)$ & 24 & 66.047 & $\mathrm{p}<.001$ & & & \\
\hline \multicolumn{7}{|l|}{ Variation du niveau général de la fluidité sociale } \\
\hline 7 - Différence uniforme & 192 & 364.810 & $\mathrm{p}<.001$ & 2.7 & 97.9 & -1648.1 \\
\hline 8 - Différence uniforme avec tendance linéaire & 195 & 365.615 & $\mathrm{p}<.001$ & 2.7 & 97.9 & -1678.8 \\
\hline Différence (2) - (8) & 1 & 35.643 & $\mathrm{p}<.001$ & & & \\
\hline Différence $(8)-(7)$ & 3 & 0.805 & ns & & & \\
\hline \multicolumn{7}{|c|}{ Variation de la propension à l'immobilité et du niveau général de la fluidité sociale } \\
\hline 9 - Combinaison des modèles 4 et 8 & 194 & 364.342 & $\mathrm{p}<.001$ & 2.7 & 97.9 & -1669.6 \\
\hline Différence (4) - (9) & 1 & 9.737 & $\mathrm{p}<.01$ & & & \\
\hline Différence (8) - (9) & 1 & 1.273 & ns & & & \\
\hline
\end{tabular}


10 - Combinaison des modèles 6 et 8

163

254.580

$\mathrm{p}<.001$

1.9

98.5

$-1454.3$

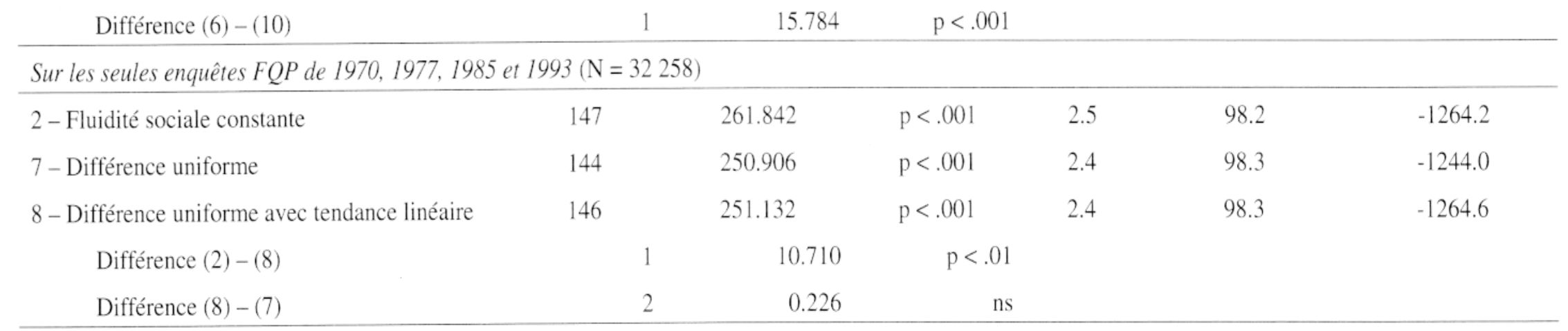

\begin{tabular}{|c|c|c|c|c|c|}
\hline \multirow{2}{*}{$\begin{array}{c}\text { Modèle } \\
3-\text { Variation commune aux classes } \\
\text { et spécifique à l'année }\end{array}$} & \multicolumn{5}{|c|}{ Paramètres estimés } \\
\hline & $\begin{array}{l}a_{1953}=0 \text { (référence) } \\
a_{1985}=-0.269^{* * *}\end{array}$ & $\begin{array}{l}a_{1970} \\
a_{1993}\end{array}$ & $\begin{array}{l}.201 * * * \\
.276 * * *\end{array}$ & $a_{1977}=-0.248 * * *$ & \\
\hline 4 - Tendance linéaire commune aux classes & $a_{\operatorname{lin}}=-0.0067 * * *$ & & & & \\
\hline 7 - Différence uniforme & $\beta_{1953}=1.000$ & $\beta_{1970}=0.905$ & $\beta_{1977}=0.872$ & $\beta_{1985}=0.851$ & $\beta_{1993}=0.806$ \\
\hline 8 - Différence uniforme avec tendance linéaire & $\beta_{\text {lin }}=-0.0046$ & & & & \\
\hline 9 - Combinaison des modèles 4 et 8 & $\alpha_{\operatorname{lin}}=-0.0020$ & \multirow{2}{*}{\multicolumn{2}{|c|}{$\beta_{\text {lin }}=-0.0037$}} & & \\
\hline 10 - Combinaison des modèles 6 et 8 & $\beta_{\text {lin }}=-0.0060$ & & & & \\
\hline \multicolumn{6}{|c|}{ Sur les seules enquêtes FQP de 1970, 1977, 1985 et $1993(\mathrm{~N}=32$ 258) } \\
\hline 7 - Différence uniforme & $\beta_{1970}=1.000$ & $\beta_{1977}=0.967$ & $\beta_{1985}=0.944$ & $\beta_{1993}=0.897$ & \\
\hline 8 - Différence uniforme avec tendance linéaire & $\beta_{\operatorname{lin}}=-0.0042$ & & & & \\
\hline
\end{tabular}


Femmes $(N=18484)$

\begin{tabular}{|c|c|c|c|c|c|c|}
\hline Modèle & $\begin{array}{l}\text { Degrés de } \\
\text { liberté }\end{array}$ & Khi-deux & Test & Delta $(\%)$ & $\begin{array}{l}\text { Part de khi-deux } \\
\text { expliquée }(\%)\end{array}$ & BIC \\
\hline 1 - Fluidité parfaite à chaque date & 245 & 6964.391 & $\mathrm{p}<.001$ & 22.5 & - & 4557.3 \\
\hline 2 -Fluidité sociale constante & 184 & 341.859 & $\mathrm{p}<.001$ & 3.6 & 95.1 & -1465.9 \\
\hline \multicolumn{7}{|l|}{ Variation de la propension à l'immobilité } \\
\hline $\begin{array}{c}3 \text { - Variation commune aux classes } \\
\text { et spécifique à l'année }\end{array}$ & 180 & 316.361 & $\mathrm{p}<.001$ & 3.5 & 95.5 & -1452.1 \\
\hline 4 - Tendance linéaire commune aux classes & 183 & 324.174 & $\mathrm{p}<.001$ & 3.6 & 95.3 & -1473.7 \\
\hline Différence (2) - (4) & 1 & 17.685 & $\mathrm{p}<.001$ & & & \\
\hline Différence (4) - (3) & 3 & 7.813 & $\mathrm{p}<.10$ & & & \\
\hline 5 - Tendance linéaire spécifique à chaque classe & 176 & 310.536 & $\mathrm{p}<.001$ & 3.5 & 95.5 & -1418.6 \\
\hline Différence (4) - (5) & 7 & 13.638 & $\mathrm{p}<.10$ & & & \\
\hline $\begin{array}{l}6 \text { - Variation spécifique à chaque classe } \\
\text { et à chaque date }\end{array}$ & 152 & 240.203 & $\mathrm{p}<.001$ & 2.7 & 96.6 & -1253.1 \\
\hline Différence (5) - (6) & 24 & 70.333 & $\mathrm{p}<.001$ & & & \\
\hline \multicolumn{7}{|l|}{ Variation du niveau général de la fluidité sociale } \\
\hline 7 - Différence uniforme & 180 & 312.914 & $\mathrm{p}<.001$ & 3.5 & 95.5 & -1455.5 \\
\hline 8 - Différence uniforme avec tendance linéaire & 183 & 320.250 & $\mathrm{p}<.001$ & 3.5 & 95.4 & -1477.7 \\
\hline Différence (2) - (8) & 1 & 21.609 & $\mathrm{p}<.001$ & & & \\
\hline Différence (8) - (7) & 3 & 7.336 & $\mathrm{p}<.10$ & & & \\
\hline \multicolumn{7}{|c|}{ Variation de la propension à l'immobilité et du niveau général de la fluidité sociale } \\
\hline 9 - Combinaison des modèles 4 et 8 & 182 & 318.448 & $\mathrm{p}<.001$ & 3.5 & 95.4 & -1469.6 \\
\hline Différence (4) - (9) & 1 & 5.726 & $\mathrm{p}<.02$ & & & \\
\hline Différence (8) - (9) & 1 & 1.802 & ns & & & \\
\hline
\end{tabular}

$$
\text { Différence (2) - (4) }
$$$$
\text { Différence (4) - (5) }
$$$$
\text { et à chaque date }
$$ 
10 - Combinaison des modèles 6 et 8

151

234.761

$\mathrm{p}<.001$

2.7

96.6

$-1248.8$

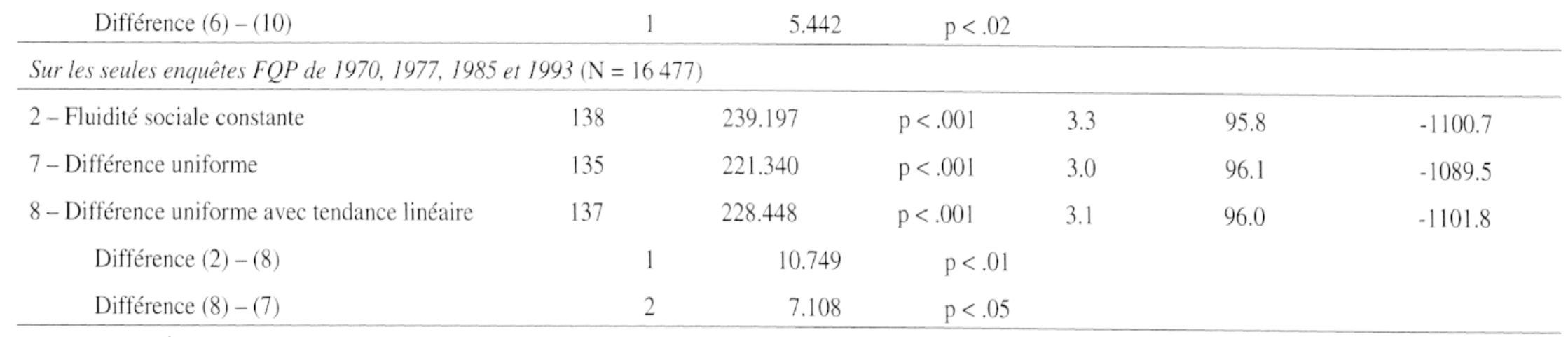

Le nombre de degrés de liberté de chaque modèle est éventuellement ajusté pour tenir compte de la présence de zéros dans la marge (OP) observée (Bishop, Fienberg et Holland, 1975, pp. 115-119).

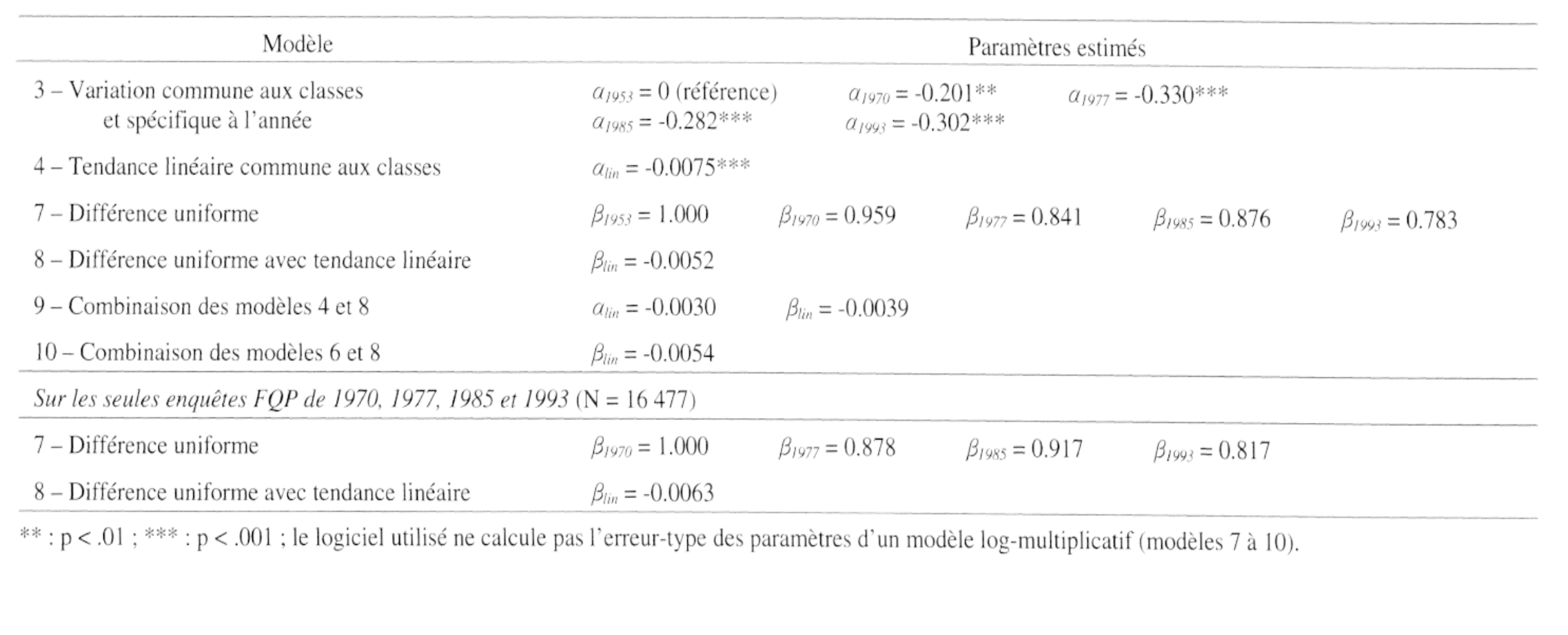


masculin, doit finalement être sélectionné. C'est enfin l'intensité de la tendance régulière à l'augmentation de la fluidité sociale qui ne diffère pas de celle mesurée sur les hommes : le paramètre $\beta_{l i n}$ vaut $-0,0052$ et exprime ainsi un rythme annuel de $0,5 \%$ pour la diminution de la force du lien entre la position sociale des femmes et celle de leur père. En vérité, la seule différence qui sépare l'analyse des tables féminines de celle conduite pour les hommes porte sur le modèle UNIDIFF dans sa forme la plus générale (modèle 7) : une non-linéarité apparaît lorsque, aux trois tables construites dans la version CSP de notre nomenclature, succèdent celles qui utilisent la version PCS; $\beta_{1985}$ est en effet supérieur à $\beta_{1977}$. Nous sommes cependant enclin à minorer l'importance de cette irrégularité et à la tenir pour un artefact créé par le changement de codification puisque rien d'équivalent n'apparaissait, dans les pages précédentes, quand l'analyse était conduite, dans une nomenclature homogène, sur la période 1977-1993. La présence et la direction de cet artefact signaleraient ainsi qu'en mêlant dans une analyse unique des tables de nomenclatures quelque peu différentes, nous sous-évaluons en réalité légèrement l'amplitude du changement intervenu en quarante ans. La comparaison, pour les hommes et les femmes, de l'estimation produite par l'analyse générale - une diminution de la force du lien d'environ $20 \%$ - avec celle qui dérive de l'examen séparé des deux périodes (1953-1977 et 1977-1993) - une diminution d'environ $25 \%$ d'après les résultats d'une section précédente - accrédite d'ailleurs ce diagnostic (33).

De l'analyse générale découlent en définitive trois enseignements majeurs. Premièrement, un modèle particulièrement simple qui ne diffère de celui d'invariance temporelle que par l'estimation d'un paramètre supplémentaire décrit mieux que ce dernier les tables de mobilité observées en France pour les hommes et les femmes sur une période de quarante ans. Il révèle une transformation unidirectionnelle de la société française dans le sens d'une fluidité accrue, c'est-à-dire d'une diminution régulière de l'inégalité des chances sociales, et l'estimation de modèles différents confirme la robustesse et l'amplitude de ce mouvement. Il faudra donc, avant la fin de cet article et par des moyens appropriés, donner à voir la circulation sociale supplémentaire que l'existence d'une telle tendance a impliquée. Deuxièmement, les recherches antérieures portant sur la période 1953-1977 (Goldthorpe, Portocarero, 1981 ; Thélot, 1982 ; Vallet, 1991, 1992) interprétaient comme une diminution de la seule propension à l'immobilité ce qui n'était en réalité que la partie la plus immédiatement visible d'un mouvement plus général d'ouverture du régime de mobilité. Ce dernier n'était en effet guère décelable car les outils nécessaires pour le mettre en évidence - notamment le modèle UNIDIFF - n'étaient pas disponibles au moment où ces travaux étaient conduits. Troisièmement, on pouvait craindre que les

(33) On en trouve enfin une preuve en estimant le modèle UNIDIFF sur les tables de mobilité de 1977 présentées dans les versions CSP et PCS de notre nomenclature. En fixant $\beta_{C S P}$ à $1, \beta_{P C S}$ vaut 1,069 parmi les femmes et 1,040 parmi les hommes. L'association statistique entre origine et po- sition sociales apparaît donc plus forte dans la version PCS. Il s'ensuit que l'ampleur de l'évolution de long terme est un peu sous-estimée dès que des tables de mobilité utilisant les deux versions sont mêlées. 
données de 1953 pèsent fortement dans la définition de la tendance observée et obscurcissent ainsi notre vision de la période étudiée. Une recherche antérieure avait en effet suggéré que ces données - les plus anciennes - étaient peut-être assez peu comparables aux autres (Thélot, 1983). Pour ce qui concerne notre entreprise au moins, cette crainte n'était pas fondée : comme le montrent les Tableaux VIII et IX, l'ampleur de la tendance annuelle ne varie guère pour les hommes et est même amplifiće pour les femmes lorsque les seules enquêtes Formation - Qualification Professionnelle de 1970 à 1993 sont prises en considération.

\section{Une ultime confirmation à l'aide du modèle log-multiplicatif d'association de Goodman}

En suivant une démarche étroitement inspirée de celle qu'adoptaient Robert Erikson et John Goldthorpe dans The constant flux, nous sommes ainsi parvenu, pour le cas de la France, à une situation que les auteurs eux-mêmes considéraient comme critique pour la thèse de l'invariance temporelle du régime de mobilité entre générations. Après avoir introduit le modèle UNIDIFF et celui autorisant une variation entre cohortes dans la propension à l'immobilité, ils signalaient en effet qu'ils ne testeraient pas la version contrainte de ces modèles - celle incluant une tendance monotone - mais ajoutaient aussitôt qu'à supposer que cette forme de variation apparaisse, il serait imprudent de la négliger, et cela même dans le cas où elle ne se manifesterait qu'à la limite de la significativité statistique (34). Or, c'est bien une telle variation, dotée en outre d'une forte significativité, que nous avons mise au jour, pour les hommes et les femmes, dans la France de la seconde moitié du siècle.

On se rappelle que la recherche de Ganzeboom, Luijkx et Treiman (1989) comme celle de Wong (1994) faisaient usage du modèle log-multiplicatif d'association proposé par Goodman (1979) et nous l'emploierons ici, à titre d'ultime confirmation de notre résultat, sur les données françaises. Dans le cadre de l'analyse de la mobilité entre générations, un tel modèle peut être interprété dans les termes suivants : dans la génération des pères comme dans celle des fils (ou filles), la structure sociale forme une échelle pour laquelle on peut, simultanément, déterminer l'ordre des barreaux et mesurer les intervalles qui les séparent. Ce modèle a donc l'intérêt de ne reposer sur aucune hypothèse a priori concernant l'ordre des catégories dans la structure sociale et ce dernier apparaît comme un produit de l'analyse. En vérité, nous n'entendons pas adopter

(34) «Since we still do not test quite specifically for monotonic change, we show here for each of our nations both the improvement in fit produced by these models and the uniform-or diagonal-change parameters that are estimated for each cohort. Should the latter indicate monotonic change, it would be unwise to discount this, even if the improvement in fit falls short of significance. " (Erikson, Goldthorpc, 1992, pp. 9394). 
cette vision de la structure sociale comme un ordre total et partageons en réalité beaucoup plus la conception d'Erikson et Goldthorpe d'un ensemble de classes discrètes entre lesquelles seuls certains ordres partiels peuvent être déterminés. Aussi notre usage du modèle de Goodman sera-t-il «faible », i.e. nous l'emploierons comme un pur outil statistique en vue de tester, une dernière fois, la robustesse de notre résultat. Appliqué à un ensemble de tables recueillies à des dates différentes, ce modèle peut s'écrire, dans sa version la plus générale :

$$
\log \left(m_{i j t}\right)=\lambda+\lambda_{i}^{O}+\lambda_{j}^{P}+\lambda_{t}^{T}+\lambda_{i t}^{o T}+\lambda_{j t}^{P T}+\phi_{t} \mu_{i t} \nu_{j t}
$$

et implique :

$$
\log (\text { od })=\phi_{t}\left(\mu_{i t}-\mu_{i^{\prime} t}\right)\left(v_{j t}-v_{j^{\prime} t}\right)
$$

L'association statistique entre origine et position sociales est donc analysée sous la forme de trois ensembles de paramètres. Les paramètres $\mu_{i t}$ (ou échelle des scores d'origine) expriment la position de la catégorie $i$ considérée sur l'échelle estimée pour les classes d'origine à la date $t$. Les paramètres $v_{j t}$ (ou échelle des scores de destination) font de même s'agissant de l'échelle estimée, à la même date, pour les classes de destination. Ainsi et comme l'indique le logarithme du odds ratio, plus les catégories $i$ et $i$ 'd'une part, $j$ et $j$ ' d'autre part sont distantes, plus la concurrence est inégale entre les individus des deux origines pour atteindre l'une plutôt que l'autre des deux positions. Dans ce cadre, le paramètre $\phi_{l}$ fournit alors une mesure générale de la force du lien entre origine et position à la date $t$.

Sous cette forme, le modèle de Goodman s'ajuste médiocrement à des données de mobilité réelles - qu'elles soient françaises ou étrangères - car il reconstruit mal l'immobilité sociale, variable d'une classe à l'autre. Nous compléterons donc l'expression précédente par un terme $\gamma_{i} d_{i j}$ où $d_{i j}$ vaut 1 si $i$ est égal à $j$ et 0 sinon; le paramètre $\gamma_{i}$ traduira alors la propension à l'immobilité - invariable dans le temps - de la classe $i$. Par ailleurs, le modèle de Goodman peut être envisagé sous des formes plus contraignantes (Becker, Clogg, 1989) et nous supposerons ici que l'échelle estimée pour les classes d'origine et celle relative aux classes de destination sont inchangées d'une enquête à l'autre. Concentrant ainsi la variation temporelle dans le seul paramètre $\phi$, il est alors possible d'estimer le modèle sous trois formes distinctes. La première postule l'identité des $\phi_{t}$, c'est-à-dire l'invariance temporelle de la force du lien entre milieu d'origine et position occupée :

$$
\log \left(m_{i j t}\right)=\lambda+\lambda_{i}^{O}+\lambda_{j}^{P}+\lambda_{t}^{T}+\lambda_{i t}^{O T}+\lambda_{j t}^{P T}+\gamma_{i} d_{i j}+\phi \mu_{i} v_{j}
$$

(Modèle 11)

La seconde autorise l'hétérogénéité des $\phi_{t}$, c'est-à-dire la modification quelconque de la force du lien entre origine et position au cours du temps :

$$
\log \left(m_{i j t}\right)=\lambda+\lambda_{i}^{O}+\lambda_{j}^{\prime}+\lambda_{t}^{T}+\lambda_{i t}^{o T}+\lambda_{j t}^{\prime T}+\gamma_{i} d_{i j}+\phi_{1} \mu_{i} v_{j}
$$


La troisième contraint enfin cette variation temporelle à suivre une tendance linéaire (Wong, 1995) :

$$
\log \left(m_{i j t}\right)=\lambda+\lambda_{i}^{O}+\lambda_{j}^{P}+\lambda_{t}^{T}+\lambda_{i t}^{O T}+\lambda_{j t}^{P T}+\gamma_{i} d_{i j}+\phi_{0}\left(1+\phi_{\text {lin }} \text { year }\right) \mu_{i} v_{j}
$$

(Modèle 13)

où year est la variable quantitative définie précédemment.

Dans le cadre du modèle de Goodman qui envisage le lien entre origine et position sociales sous une forme très différente de celle du modèle UNIDIFF, une confirmation stricte de notre résultat pourra être obtenue si : premièrement, les paramètres $\phi_{t}$ estimés sous le modèle 12 diminuent régulièrement de 1953 à 1993 ; deuxièmement, cette variation peut être résumée, sans perte d'information notable, par la tendance linéaire du modèle 13; troisièmement, cette dernière s'avère statistiquement significative et le modèle de Goodman muni d'une tendance linéaire fournit, en outre, une statistique BIC qui conduit à le préférer à celui d'invariance (modèle 11 ). Les résultats de nos estimations sont présentés dans le Tableau X.

Ces trois prédictions sont étroitement réalisées, en dehors d'une irrégularité, mineure et déjà apparue, liée au fait que, pour les femmes, $\phi_{1985}$ est supérieur -

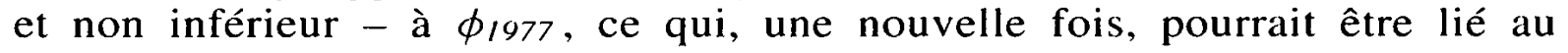
changement de nomenclature intervenu entre les deux enquêtes. On relèvera que $\phi_{\text {lin }}$ vaut $-0,0080$ parmi les hommes et $-0,0073$ parmi les femmes. Ce rythme annuel de variation pour la diminution de la force du lien entre milieu d'origine et position occupée est très proche de celui estimé par Ganzeboom, Luijkx et Treiman (1989) à l'aide du même modèle - environ un pour cent par an. Il est identique à celui obtenu par Wong (1994) dans le cas des États-Unis entre 1947 et 1986 . Il est en revanche supérieur à celui que révélait, sur les données françaises, le modèle UNIDIFF - environ un demi pour cent par an. Il serait toutefois imprudent de réviser nos estimations à la hausse puisque, comme l'indiquent les valeurs de khi-deux obtenues, le modèle d'association de Goodman décrit en réalité moins fidèlement que le modèle UNIDIFF les tables de mobilité françaises. Cela confirme au demeurant les réserves qu'exprimaient Erikson et Goldthorpe (1992, p. 100) à l'égard des modèles d'association : ils semblent en effet surestimer l'ampleur de l'évolution intervenue.

Bref, un modèle très différent de celui utilisé initialement confirme que la fluidité sociale s'est accrue continûment, au sein de la société française, durant quatre décennies. Et, autre aspect important, la même évolution peut être mise au jour à partir de données différentes de celles des enquêtes Formation Qualification Professionnelle : les enquêtes Emploi de 1982 et 1997 (35).

(35) Au cours des échanges que nous avons eus avec lui durant la préparation de cet article, Michel Forsé a estimé le modèle unIDIFF sur deux tables de mobilité père-fils, issues des enquêtes Emploi de 1982 et 1997, portant sur des hommes actifs âgés de 40 à 55 ans et construites dans la nomenclature des PCS à un chiffre $(\mathrm{N}=5107$ pour chaque table). Fixé à 1 en 1982, le paramètre $\beta$ vaut 0,92 en 1997 , indiquant ainsi une augmentation de la fluidité sociale de $8 \%$ en 15 ans, soit de nouveau $0,5 \%$ par an. Cette différence est en outre significative au seuil de dix pour cent. Nous remercions Michel Forsé d'a voir mis ses résultats à notre disposition (Forsé, 1998). 


\begin{tabular}{|c|c|c|c|c|c|c|}
\hline Modèle & $\begin{array}{l}\text { Degrés de } \\
\text { liberté }\end{array}$ & Khi-deux & Test & Part de $\mathrm{k}$ & $\begin{array}{l}\text { hi-deux expliquée } \\
(\%)\end{array}$ & $\mathrm{BIC}$ \\
\hline \multicolumn{7}{|l|}{ Hommes $(\mathrm{N}=35741)$} \\
\hline 11 - Invariance temporelle des $\phi$ & 224 & 939.527 & $\mathrm{p}<.001$ & 4.4 & 94.6 & -1408.9 \\
\hline 12 - Hétérogénéité des $\phi$ & 220 & 895.752 & $\mathrm{p}<.001$ & 4.3 & 94.8 & -1410.7 \\
\hline 13 - Tendance linéaire des $\phi$ & 223 & 899.698 & $\mathrm{p}<.001$ & 4.3 & 94.8 & -1438.2 \\
\hline Différence (11) - (13) & 1 & 39.829 & $\mathrm{p}<.001$ & & & \\
\hline Différence (13) - (12) & 3 & 3.946 & ns & & & \\
\hline \multicolumn{7}{|l|}{ Femmes $(\mathrm{N}=18484)$} \\
\hline $11-$ Invariance temporelle des $\phi$ & 224 & 574.787 & $\mathrm{p}<.001$ & 5.3 & 91.7 & -1625.9 \\
\hline 12 - Hétérogénéité des $\phi$ & 220 & 554.428 & $\mathrm{p}<.001$ & 5.2 & 92.0 & -1607.0 \\
\hline 13 - Tendance linéaire des $\phi$ & 223 & 560.331 & $\mathrm{p}<.001$ & 5.3 & 92.0 & -1630.6 \\
\hline Différence (11) - (13) & 1 & 14.456 & $\mathrm{p}<.001$ & & & \\
\hline Différence (13) - (12) & 3 & 5.903 & $\mathrm{~ns}$ & & & \\
\hline Modèle & \multicolumn{6}{|c|}{ Paramètres estimés } \\
\hline \multicolumn{7}{|l|}{ Hommes $(\mathrm{N}=35741)$} \\
\hline 11 - Invariance temporelle des $\phi$ & $\phi=7.651$ & & & & & \\
\hline 12 - Hétérogénéité des $\phi$ & $\phi_{1953}=9.672$ & $\phi_{1970}=7.830$ & $\phi_{1977}=7.396$ & $\phi_{1985}=7.212$ & $\phi_{1993}=6.141$ & \\
\hline $13-$ Tendance linéaire des $\phi$ & $\phi_{0}=9.300$ & $\phi_{\operatorname{lin}}=-0.0080$ & & & & \\
\hline \multicolumn{7}{|l|}{ Femmes $(\mathrm{N}=18484)$} \\
\hline 11 - Invariance temporelle des $\phi$ & $\phi=8.409$ & & & & & \\
\hline 12 - Hétérogénéité des $\phi$ & $\phi_{1953}=11.239$ & $\phi_{1970}=10.391$ & $\phi_{1977}=8.293$ & $\phi_{1985}=8.862$ & $\phi_{1993}=7.842$ & \\
\hline $13-$ Tendance linéaire des $\phi$ & $\phi_{0}=10.835$ & $\phi_{\text {lin }}=-0.0073$ & & & & \\
\hline
\end{tabular}

Le logiciel utilisé ne calcule pas l'erreur-type des paramètres d'un modèle log-multiplicatif (modèles 11 à 13 ). 


\section{Une appréhension concrète de la réduction de l'inégalité des chances sociales}

Selon le modèle qui exprime le mieux l'évolution intervenue en France en quarante ans, la force intrinsèque du lien qui unit la position sociale que les individus occupent du fait de leur emploi à leur milieu d'origine a décliné au rythme de $0,5 \%$ par an, soit une variation de $20 \%$ sur l'ensemble de la période. Cette formulation reste très abstraite et il importe désormais de prendre la mesure concrète du changement qu'a entraîné cette ouverture du régime de mobilité entre générations. $\mathrm{Si}$, en effet, la fluidité sociale s'est accrue, c'est que des hommes et des femmes occupent aujourd'hui des positions sociales qui $n$ 'auraient pas été les leurs dans le cas où le régime de mobilité serait demeuré celui de la France du milieu du siècle. Il faut donc rendre visible la circulation sociale supplémentaire qu'a entraînée la réduction légère, mais continue, de l'inégalité des chances.

Partons des tables de mobilité estimées sous le modèle UNIDIFF muni d'une tendance linéaire. Elles sont établies sur un échantillon. À l'aide d'une multiplication par une constante appropriée, nous pouvons en premier lieu ajuster à celle de 1993 les marges caractéristiques de la société française à cette date, c'est-à-dire la distribution socioprofessionnelle des hommes (ou femmes) français de 35 à 59 ans qui occupent un emploi et celle de leurs pères. Nommons $T$ le tableau ainsi obtenu. Il ne diffère guère de la table réelle de 1993 - celle directement extrapolée des données de l'enquête - puisque le modèle fournit une description assez fidèle de la période étudiée. Prenons en second lieu la table de mobilité estimée en 1953. En lui ajustant les marges caractéristiques de la société française en 1993, nous obtenons une table contrefactuelle, celle que l'on aurait dû observer en 1993 si le régime de mobilité était demeuré strictement celui de la France du début des années cinquante. Il suffit pour cela de faire usage d'un algorithme dont la caractéristique essentielle est de préserver les odds ratios, c'est-à-dire la structure de la mobilité, de la table-échantillon (36). Si l'on convient de nommer $T$ ' le tableau ainsi construit, il reste alors à calculer, cellule par cellule, la différence $T$ - T' pour rendre visible la circulation sociale qui, en 1993, a résulté de la seule ouverture du régime de mobilité entre générations. Ce sont précisément ces différences, arrondies au millier, qui sont présentées dans le Tableau XI. Tout en fournissant un aperçu plausible de l'accroissement de la fluidité sociale, elles sont aussi entachées d'une certaine incertitude puisque tout le calcul est fondé sur les estimations d'un modèle. Il convient donc de ne pas accorder une importance démesurée aux faibles effectifs. Il est aussi souhaitable de s'assurer, sur des populations distinctes, de la solidité de la configuration obtenue.

(36) Cet algorithme a déjà été évoqué dans la note 9 du présent article. 
Considérons d'abord la première table, relative aux hommes français de 35 à 59 ans qui ont un emploi en 1993. Par construction, le tableau des différences a, aux arrondis près, une somme nulle pour chaque ligne et chaque colonne. En effet, la circulation sociale qui résulte de la seule ouverture du régime de mobilité entre générations peut être décrite ainsi : parmi les hommes d'une origine donnée, il en est qui n'occupent pas certaines positions, mais en ont rejoint d'autres, lesquelles ne peuvent alors être tenues par les hommes qui ont grandi dans un autre milieu. L'ouverture du régime de mobilité entre générations s'apparente ainsi à un jeu à somme nulle où les positions gagnées par certains ont été perdues par d'autres. Que peut-on dès lors observer?

Le mouvement prend naissance dans la chute de l'immobilité. La diagonale ne porte en effet que des effectifs négatifs, même si ces derniers apparaissent aussi - moins fréquemment - dans d'autres secteurs de la table. À partir de là, les destins sociaux diffèrent selon le milieu d'origine considéré, mais c'est bien d'une réduction de l'inégalité des chances qu'il s'agit. Les fils d'agriculteur qui, en raison de l'accroissement de la fluidité sociale, ne sont pas à la terre ont surtout rejoint des positions de cadre, supérieur ou moyen. Il en va de même des fils des salariés de l'agriculture qui n'ont pas suivi les traces de leur père et ne sont pas non plus devenus ouvriers. Des enfants de la petite bourgeoisie indépendante ont, du fait de la seule ouverture du régime de mobilité, essaimé dans d'autres catégories sociales; ils ont surtout rejoint la classe ouvrière, signe d'une «fragilisation» de ce milieu d'origine. Le maintien dans la catégorie paternelle est devenu moins assuré parmi les fils des cadres supérieurs et moyens comme le sont aussi les chances des enfants de cadre subalterne de s'élever dans la hiérarchie sociale. Ce sont enfin 71000 fils de contremaître ou d'ouvrier qui, du seul fait de l'ouverture du régime de mobilité entre générations, ont quitté la classe ouvrière et, parmi eux, 34000 sont devenus cadres supérieurs. Au total et dans la population considérée, on peut estimer à près de 265000 , soit $3,8 \%$ de l'effectif total, le nombre d'hommes occupant des positions qui n'auraient pas été les leurs en l'absence d'une augmentation de la fluidité sociale en quarante ans. Cette proportion n'est certes pas considérable, mais on ne saurait non plus la tenir pour négligeable ou anodine.

Réalisé de manière indépendante sur la population des femmes françaises de 35 à 59 ans qui ont un emploi en 1993, le même calcul conduit à la seconde table. Il est frappant de constater à quel point la configuration qu'elle révèle est semblable à celle qui vient d'être décrite, attestant ainsi de la cohérence de notre résultat. La seule nuance réside dans le fait que la réallocation des positions sociales met en jeu plus souvent la catégorie des employés dont on sait qu'elle concentre une part importante de la main-d'œuvre féminine. Ce sont ici 20000 filles de contremaître ou d'ouvrier qui ne sont pas elles-mêmes ouvrières et 41000 qui ne sont pas devenues employées du seul fait de l'évolution intervenue en France entre 1953 et 1993 ; parmi ces femmes, 22000 occupent des positions de cadre supérieur et 17000 sont cadres moyens. Au total, on peut estimer à 195000 , soit $3,6 \%$ de l'effectif total, le nombre de femmes dont, en 1993, le destin social a été ainsi transformé. 
Louis-André Vallet

TABLEAU XI. - Circulation sociale résultant de l'ouverture du régime de mobilité entre générations (les effectifs sont exprimés en milliers d'individus)

Effet de l'ouverture sur la période 1953-1993 (Hommes français, actifs occupés, âgés de 35 à 59 ans)

\begin{tabular}{|c|c|c|c|c|c|c|c|c|c|}
\hline Père & 1 & 2 & 3 & 4 & 5 & 6 & 7 & 8 & $\begin{array}{c}\text { Total } \\
\text { (en milliers) }\end{array}$ \\
\hline I Agriculteur exploitant & -28 & -1 & +1 & +4 & +14 & +12 & 0 & -1 & $\left(\begin{array}{llll}1 & 1 & 10\end{array}\right)$ \\
\hline 2 Salarié agricole & +3 & -4 & 0 & +1 & +3 & +3 & 0 & -7 & $(220)$ \\
\hline 3 Artisan, petit commerçant & +5 & +1 & -34 & -2 & +4 & +4 & +2 & +21 & (778) \\
\hline $\begin{array}{l}4 \text { Industriel, gros commerçant, } \\
\text { profession libérale }\end{array}$ & +1 & 0 & +2 & -14 & -4 & +4 & +2 & +8 & $(225)$ \\
\hline 5 Cadre supérieur & +2 & 0 & +6 & -2 & -31 & +5 & +6 & +14 & $(502)$ \\
\hline 6 Cadre moyen & +1 & 0 & +6 & 0 & -15 & -14 & +3 & +20 & $(552)$ \\
\hline $\begin{array}{l}7 \text { Employé, personnel de } \\
\text { service, autre actif }\end{array}$ & +4 & +1 & +5 & +3 & -4 & -18 & -7 & +17 & $(837)$ \\
\hline 8 Contremaître, ouvrier & +12 & +3 & +14 & +10 & +34 & +3 & -6 & -71 & $(2713)$ \\
\hline Total (en milliers) & (346) & $(50)$ & $(674)$ & $(281)$ & $\left(\begin{array}{ll}1 & 145\end{array}\right)$ & $\left(\begin{array}{ll}1 & 302\end{array}\right)$ & (684) & $(2456)$ & $(6937)$ \\
\hline
\end{tabular}

Lire ainsi : Dans la population formée des hommes français de 35 à 59 ans qui ont un emploi en 1993 , on compte 2713000 fils de contremaître ou d'ouvrier ; en raison de l'augmentation de la fluidité sociale intervenue entre 1953 et 1993, 71000 ne sont pas eux-mêmes ouvriers et, parmi eux, 34000 sont cadres supérieurs.

Effet de l'ouverture sur la période 1953-1993 (Femmes françaises, actives occupées, âgées de 35 à 59 ans)

\begin{tabular}{|c|c|c|c|c|c|c|c|c|c|}
\hline Fille & 1 & 2 & 3 & 4 & 5 & 6 & 7 & 8 & $\begin{array}{c}\text { Total } \\
\text { (en milliers) }\end{array}$ \\
\hline 1 Agriculteur exploitant & -27 & 0 & -2 & +1 & +10 & +14 & +6 & -2 & $(945)$ \\
\hline 2 Salarié agricole & +2 & -1 & 0 & 0 & +2 & +4 & -5 & -3 & (198) \\
\hline 3 Artisan, petit commerçant & +5 & 0 & -12 & 0 & +1 & -6 & +5 & +7 & $(641)$ \\
\hline $\begin{array}{l}4 \text { Industriel, gros commerçant, } \\
\text { profession libérale }\end{array}$ & +1 & 0 & 0 & -3 & -5 & -1 & +6 & +1 & (142) \\
\hline 5 Cadre supérieur & +2 & 0 & +3 & -1 & -28 & -4 & +23 & +5 & $(460)$ \\
\hline 6 Cadre moyen & +1 & 0 & +2 & 0 & -5 & -15 & +12 & +5 & $(406)$ \\
\hline $\begin{array}{l}7 \text { Employé, personnel de } \\
\text { service, autre actif }\end{array}$ & +2 & 0 & +1 & +1 & +3 & -9 & -5 & +7 & (631) \\
\hline 8 Contremaître, ouvrier & +13 & +1 & +6 & +3 & +22 & +17 & -41 & -20 & (1 977) \\
\hline Total (en milliers) & $(263)$ & (18) & $(330)$ & $(67)$ & $(556)$ & $(1241)$ & $(2270)$ & $(654)$ & (5 399) \\
\hline
\end{tabular}

Lire ainsi: Dans la population formée des femmes françaises de 35 à 59 ans qui ont un emploi en 1993 , on compte 1977000 filles de contremaître ou d'ouvrier ; en raison de l'augmentation de la fluidité sociale intervenue entre 1953 et 1993, 20000 ne sont pas elles-mêmes ouvrières, 41000 ne sont pas employées ou personnels de service et, parmi ces femmes, 22000 sont cadres supérieurs et 17000 sont cadres moyens. 
Revue française de sociologie

Effet de l'ouverture sur la période 1977-1993

(Hommes français et étrangers, actifs ou anciens actifs, âgés de 30 à 59 ans) (Code des PCS à un chiffre)

\begin{tabular}{|c|c|c|c|c|c|c|c|}
\hline Père & 1 & 2 & 3 & 4 & 5 & 6 & $\begin{array}{c}\text { Total } \\
\text { (en milliers) }\end{array}$ \\
\hline 1 Agriculteur exploitant & -27 & +3 & +15 & +14 & +3 & -8 & (1 646) \\
\hline $\begin{array}{l}2 \text { Artisan, commerçant, } \\
\text { chef d'entreprise }\end{array}$ & +6 & -35 & 0 & +2 & +3 & +23 & (1 338) \\
\hline $\begin{array}{l}3 \text { Cadre, profession } \\
\text { intellectuelle supérieure }\end{array}$ & +3 & +7 & -43 & +10 & +6 & +17 & $(872)$ \\
\hline 4 Profession intermédiaire & +2 & +5 & -15 & -20 & 0 & +29 & (1 228) \\
\hline 5 Employé & +3 & +4 & 0 & -14 & -12 & +19 & $(1210)$ \\
\hline 6 Ouvrier & +14 & +16 & +43 & +8 & -1 & -79 & $(4340)$ \\
\hline Total (en milliers) & (436) & $\left(\begin{array}{ll}1 & 023\end{array}\right)$ & (1 749) & (2 368) & $\left(\begin{array}{ll}1 & 062\end{array}\right)$ & (3 995) & $(10633)$ \\
\hline
\end{tabular}

Lire ainsi: Dans la population formée des hommes français et étrangers de 30 à 59 ans actifs ou anciens actifs en 1993, on compte 4340000 fils d'ouvrier ; en raison de l'augmentation de la fluidité sociale intervenue entre 1977 et 1993, 79000 ne sont pas eux-mêmes ouvriers et, parmi eux, 43000 sont cadres ou professions intellectuelles supérieures.

Puisque l'augmentation de la fluidité sociale a pris la forme d'une tendance continue, ses effets peuvent être évalués sur un intervalle temporel plus court que quatre décennies. En retenant la période 1977-1993, on traitera ainsi des transformations de la France au cours, approximativement, des vingt dernières années. Faire porter l'analyse sur la population qu'étudiaient Goux et Maurin (1997a, 1997b) - les hommes français et étrangers de 30 à 59 ans actifs ou anciens actifs - présente alors plusieurs intérêts. D'une part, en ajoutant près de quatre millions d'individus aux hommes considérés dans le premier tableau, on élargit considérablement le spectre de l'analyse. D'autre part, il est possible de retenir la nomenclature, désormais habituelle, des PCS à un chiffre. Enfin, sur ces données de 1977,1985 et 1993, le modèle UNIDIFF muni d'une tendance linéaire s'ajuste remarquablement bien (37). La circulation sociale qu'a entraînée l'ouverture, de 1977 à 1993, du régime de mobilité entre générations réapparaît encore sous la même forme. Elle a concerné, è 1993, 255000 hommes, soit $2,4 \%$ de l'effectif total. La proportion est ici plus faible que précédemment car l'intervalle temporel mis en jeu est plus court.

Toutes les évaluations qui précèdent sont fondées sur les estimations d'un modèle. Il est dès lors souhaitable de les confronter à d'autres qui ne dépen-

(37) Khi-deux vaut 62,635 pour 49 degrés de liberté, delta $1,2 \%$, BIC $-448,1$ contre $-437,5$ pour le modèle de fluidité sociale constante, $\beta_{\text {lin }}$ est estimé à $-0,0081$. Le modèle est donc rejeté au seuil de $10 \%$, mais non de $5 \%$, ce qui, en raison de l'effectif mis en jeu ( $N=33602)$, est remarquable. Ces résultats peuvent être rapprochés de ceux du Tableau VII. 
draient que des données réelles. Prenons la table de mobilité observée à l'enquête de 1977 dans la nomenclature des PCS à un chiffre; on peut lui ajuster les marges de 1993 et calculer ainsi la circulation sociale supplémentaire liée à la transformation du régime de mobilité. Comparer cette évaluation sans modèle à la précédente conduit alors à deux constats principaux. D'une part, l'effet de l'ouverture du régime de mobilité entre générations est plus fort dans cette nouvelle évaluation (400000 hommes, soit $3,8 \%$ de l'effectif total); le fait que le modèle retenu sous-estime l'ampleur de l'évolution intervenue surgit donc une nouvelle fois. D'autre part, l'évaluation sans modèle fait de nouveau apparaître en les amplifiant les aspects les plus significatifs du Tableau XI : chute de l'immobilité dans les catégories salariées, destinée cadre ou profession intermédiaire plus fréquente parmi les fils d'agriculteur, échanges accrus entre la catégorie des cadres, professions intellectuelles supérieures et celle des ouvriers. Des évaluations analogues, pour les hommes et les femmes, sur la période 1953-1993 confortent enfin ces deux constats, même s'il est nécessaire de les considérer avec quelque précaution en raison du changement de nomenclature intervenu.

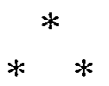

Ainsi, en France, le Flux n'a pas été constant, pour reprendre, en l'amendant, le titre du bel ouvrage que Robert Erikson et John Goldthorpe ont consacré à la mobilité sociale dans les sociétés industrielles. Dans La Seconde Révolution française où il évoquait l'augmentation de la mobilité nette que le travail de Claude Thélot mettait en évidence, Henri Mendras écrivait : «Dans les quarante dernières années, la société française s'est très visiblement assouplie. » (1988, p. 57). Et, reconnaissant qu'un changement avait pu se produire dans la période de l'après-guerre, John Goldthorpe répondait qu'il n'avait «cependant pas atteint l'intensité que le commentaire de Mendras impliquerait» (1995, p. 69). Nous savons désormais que cet assouplissement a été réel et continu, mais que le qualificatif de visible était sans doute exagéré s'agissant d'un changement qui s'effectuait à un rythme inférieur, en tout état de cause, à un pour cent par an et qui ne pouvait ainsi guère apparaître clairement, même aux yeux d'un observateur averti (38). Comme l'atteste la qualité de la description que fournit, à lui seul, le modèle de fluidité constante, les formes de l'inégalité des chances sociales sont en effet demeurées largement inchangées, mais c'est le niveau de celle-ci qui a décliné, justifiant l'expression de lente érosion que nous avons déjà employée et qui nous paraît être la plus fidèle pour traduire l'évolution intervenue (39).

(38) Au reste, la controverse scientifique aurait été moins forte si le changement avait été si visible.

(39) S'ćtonnant de n'avoir pu mettre en évidence une transformation du régime de mobilité entre 1977 et 1993, Merllié et Prévot concluaient en ces termes : «Si la mobilité observée (les flux entre catégories) changent de manière importante en même temps que la structure sociale (la taille relative des catégories), on s'attendrait plutôt à ce que le régime de mobilité (les proximités ou distances entre catégories que traduisent leurs 
Un prolongement assez immédiat du présent travail pourrait alors consister à introduire le modèle «noyau » que John Goldthorpe présentait dans cette revue dans la dynamique des tables de mobilité françaises en quatre décennies et l'on étudierait ainsi quelles dimensions du régime de mobilité ont été spécifiquement transformées. Qu'une telle évolution soit advenue en France depuis le milieu du siècle n'implique cependant ni qu'il en a été de même pour une autre société, ni que l'on puisse tenir pour assuré que le mouvement ira en se prolongeant, avant du moins que l'on ait identifié clairement quels en sont les ressorts.

Falsifier, comme nous pensons l'avoir fait dans le présent article pour lé cas de la France, la thèse de l'invariance temporelle du régime de mobilité entre générations implique, selon nous, que l'on reconnaisse que le niveau de l'inégalité des chances inscrite au cœur de la structure d'une société moderne n'est pas fixe, c'est-à-dire qu'entre les individus de différentes classes d'origine, les inégalités d'accès aux diverses positions sociales ne sont pas reproduites inexorablement d'une génération à la suivante, et donc que les sociétés et leurs membres disposent d'une latitude d'action, même limitée, pour infléchir le cours des destins sociaux des individus. Il reste cependant vrai que l'inégalité des chances est dotée d'une inertie considérable, ce qui impose alors au sociologue de recourir à des méthodes sophistiquées pour révéler sa dynamique et ses transformations. Mais réfuter la thèse de l'invariance temporelle n'implique pas ipso facto que l'on doive adopter la thèse de l'industrialisme ou sa variante postindustrielle (40), selon nous trop téléologiques et finalistes, qui conduisent à prédire la diminution régulière et inéluctable de l'inégalité des chances sociales avec l'avènement d'une Nouvelle société. Comme le soulignait Raymond Boudon en 1984, il y a place pour le désordre dans les théories du changement social.

À nos yeux, le résultat de cette recherche pourrait avoir des conséquences quant à notre compréhension de l'évolution de la société française dans la seconde moitié du siècle. L'innovation méthodologique, due à Erikson et Goldthorpe et à Xie, qui consiste, pour une relation statistique dotée d'une très forte inertie, à étudier son interaction avec le temps sous une forme log-multiplicative se révèle être un instrument très puissant pour mettre au jour des changements non détectés jusqu'alors. Et il pourrait être utile d'appliquer cette méthodologie à l'inégalité des chances scolaires pour confirmer ou infirmer la

(suite de la note 39)

échanges) en soit également affecté, à travers des modifications de la valeur sociale relative des différentes catégories. » (1997, pp. 56-58). En suivant ce commentaire (voir aussi Merllié, 1994, pp. 205-222), on peut voir le changement que nous avons mis au jour - notamment les fils et filles d'ouvrier «supplémentaires» qui sont devenus cadres - comme le résultat d'une transformation des distances entre catégories sociales liée à la croissance du groupe des cadres. Cela ne signifie évidemment pas que ce changement puisse être considéré comme un artefact ou une illusion. Signalons enfin que la modification des distances entre catégories sociales pourrait être abordée comme problème empirique à l'aide du modèle d'association de Goodman ou de ses extensions à plusieurs dimensions.

(40) Dans son ouvrage de 1973, Bell écrit ainsi que «la société postindustrielle, dans sa logique initiale, est une méritocratie ». La section Meritocracy and equality des conclusions d'où est tirée cette phrase (p. 409) n'a pas été traduite dans l'édition française. 
conclusion de stabilité à laquelle parviennent les recherches récentes. Une limite évidente du présent article est qu'en concentrant l'effort sur la mise en évidence d'une tendance de long terme et sur le test de sa robustesse, aucune hypothèse n'a été formulée, ni a fortiori éprouvée, pour expliquer le mouvement mis au jour. C'est à cette tâche qu'il faut désormais s'atteler. Entre le milieu d'origine et la position occupée s'intercalent les études accomplies dans le système scolaire et l'expérience accumulée dans le parcours professionnel. Et l'enquête sur l'emploi de 1953 comme les enquêtes Formation - Qualification Professionnelle de 1964 à 1993 contiennent les informations nécessaires pour tester diverses hypothèses relatives aux changements qui auraient pu advenir dans les relations intermédiaires.

À cet égard, s'il ne fait guère de doute que la croissance de la scolarisation et la démocratisation de l'enseignement sont incapables d'introduire rapidement des transformations fortes dans la structure de la mobilité, faut-il tenir pour assuré qu'elles n'entraînent, sur le long terme, aucun changement? Car c'est bien une transformation unidirectionnelle, ténue mais continue, vers une fluidité accrue que nous avons mise au jour, pour les hommes et les femmes, sur une période de quarante ans. Il est ici stimulant de relire L'inégalité des chances en gardant à l'esprit ce qu'a trouvé Michael Hout pour les États-Unis. Dans la société américaine, l'association statistique entre milieu d'origine et position occupée est d'autant plus faible que l'on considère des individus plus instruits. Ainsi, en l'absence de toute modification de l'inégalité des chances scolaires comme du mécanisme d'allocation des positions sociales en fonction du diplôme, l'élévation générale du niveau d'éducation peut à elle seule affaiblir, par un effet de composition, la relation entre origine et position sociales. Il faudra encore, en suivant un travail récent de Chauvel (1997), étudier plus précisément quelle a été la variation de la fluidité sociale au fil des cohortes de naissance. Il reste ainsi beaucoup à faire pour comprendre quels ont été les ressorts de la dynamique de l'inégalité des chances sociales dans la France de la seconde moitié du $\mathrm{XX}^{\mathrm{e}}$ siècle.

Louis-André VALLET

LASMAS-Institut du Longitudinal - CNRS Membre associé du LSQ-CREST Maison de la Recherche en Sciences Humaines - Université de Caen Esplanade de la Paix, 14032 Caen Cedex vallet@mrsh.unicaen.fr 


\section{RÉFÉRENCES BIBLIOGRAPHIQUES}

Becker M. P., Clogg C. C., 1989. - « Analysis of sets of two-way contingency tables using association models », Journal of the American statistical association, 84, 405, pp. 142-151.

Bell D., 1973. - The coming of post-industrial society. A venture in social forecasting, New York, Basic Books [Trad. fr. 1976, Paris, Laffont].

Bertaux D., 1969. - «Sur l'analyse des tables de mobilité sociale », Revue française de sociologie, IO, 4, pp. 448-490.

Bishop Y. M. M., Fienberg S. E., Holland P. W., 1975. - Discrete multivariate analysis : theory and practice, Cambridge, MIT Press.

Blau P. M., Duncan O. D., 1967. - The American occupational structure, New York, Wiley.

Boudon R., 1973. - L'inégalité des chances. la mobilité sociale dans les sociétés industrielles, Paris, Armand Colin, [2 éd. 1978, $3^{\circ}$ éd. 1985].

- 1984. - La place du désordre. Critique des théories du changement social, Paris, Presses Universitaires de France.

Brésard M., 1950. - «Mobilité sociale et dimension de la famille», Population, 5, 3, pp. 533-566.

Cautrès B., 1997. - «Modèles log-linéaires et analyse comparative des données d'enquêtè ", Revue internationale de politique comparée, 4, 1, pp. 71-112.

Chauvel L., 1997. - Évolution du système de stratification sociale et succession des cohortes, Thèse de doctorat de l'Université de Lille I, sous la direction de Michel Forsé.

Chenu A., 1990. - L'archipel des employés, Paris, INSEE (Études).

Cherkaoui M., 1992. - «Mobilité» dans R. Boudon (éd.), Traité de sociologie, Paris, Presses Universitaires de France, pp. 153-193.

Clogg C. C., Shihadeh E. S., 1994. - Statistical models for ordinal variables, London, Sage.

Cuin C.-H., 1993. - Les sociologues et la mobilité sociale, Paris, Presses Universitaires de France.

Desabie J., 1954. - «L'enquête par sondage sur l'emploi de juin 1953 », Bulletin mensuel de statistique. nouvelle séric, supplément octobre-décembre, pp. 32-40.

- 1955. - «La mobilité sociale en France », Bulletin de la sFIIIS, $1^{\mathrm{or}}$ novembre.

Duncan O. D., 1966. - «Mcthodological issues in the analysis of social mobility » dans N. J. Smelser, S. M. Iipset (eds.), Social structure and mobility in economic development, Chicago, Aldine, pp. $51-97$.

Erikson R., 1983. - « Changes in social mobility in industrial nations : the case of Sweden », Research in social stratification and mobility, 2, pp. 165-195.

Erikson R., Goldthorpe J. H., 1992. - The constant flux. A study of class mobility in industrial societies, Oxford, Clarendon Press.

Erikson R., Goldthorpe J. H., Portocarero L., 1979. - «Intergenerational class mobility in three Western European societies: England, France and Sweden », British journal of sociology, 30, 4, pp. 415-441.

Featherman D. L., Hauser R. M., 1978. - Opportunity and change, New York, Academic Press.

Forsé M., 1997. - «La diminution de l'inégalité des chances scolaires ne suffit pas à réduire l'inégalité des chances sociales », Revue de l'OFCF, 63, pp. 229-239.

- 1998. - «French trends in social and educational opportunitics, 1982-1997», The Tocqueville review, 19. 1, pp. 173-186.

Forsé M., Chauvel L., 1995. - «L'évolution de l'homogamie en France. Une méthode pour comparer les diagonalités de plusieurs tables », Revue française de sociologie, 36, 1, pp. 123-142.

Fukumoto I. K., Grusky D. B., 1993. - « Social mobility and class structure in early-industrial France » dans A. Miles, D. Vincent (eds.), Building European society. Occupational change and social mobility in Europe, 1840-1940, Manchester, Manchester University Press, pp. 40-67. 
Ganzeboom H. B. G., De Graaf P., 1984. - «Intergenerational occupational mobility in the Netherlands in 1954 and 1977 : a loglinear analysis " dans B. F. M. Bakker, J. Dronkers, H. B. G. Ganzeboom (eds.), Social stratification and mobility in the Netherlands, Amsterdam, siswo, pp. 71-90.

Ganzeboom H. B. G., Luijkx R., Róbert P., 1989. - «Trends in intergenerational occupational mobility in Hungary between 1930 and 1989» dans R. Iuijkx, 1994, Comparative loglinear analyses of social mobility and heterogamy, Tilburg, Tilburg University Press, pp. 43-75.

Ganzeboom H. B. G., Luijkx R., Treiman D. J., 1989. - «Intergenerational class mobility in comparative perspective », Research in social stratification and mobility, 8, pp. 3-84.

Goldthorpe J. H., 1980. - Social mobility and class structure in modern Britain, Oxford, Clarendon Press [2" éd. 1987].

- 1995. - «Le "noyau dur" : fluidité sociale en Angleterre et en France dans les années 70 et 80 », Revue française de sociologie, 36, 1, pp. 61-79.

Goldthorpe J. H., Payne C., 1986. - « Trends in intergenerational class mobility in England and Wales, 1972-1983», Sociology, 20, 1, pp. 1-24.

Goldthorpe J. H., Portocarero I.., 1981. - «La mobilité sociale en France, 1953-1970: un nouvel examen », Revue française de sociologie, 22, 2, pp. $151-166$.

Gollac M., Laulhé P., 1987. - «La transmission du statut social. L'échelle et le fossé », Économie et statistique, 199-200, pp. 85-93.

Goodman L. A., 1972. - «A general model for the analysis of surveys », American journal of sociology, 77, 6, pp. 1035-1086.

- 1979. - « Simple models for the analysis of association in cross-classifications having ordered categories », Journal of the American statistical association, 74, 367, pp. 537-552.

Goux D., Maurin É., 1997a. - « Destinées sociales : le rôle de l'école et du milieu d'origine »,Économie et statistique, 306, pp. 13-26.

- 1997b. - «Meritocracy and social heredity in France : some aspects and trends», European sociological review, 13, 2, pp. 159-177.

Hagenaars J. A., 1990. - Categorical longitudinal data. Log-linear panel, trend, and cohort analysis, London, Sage.

Hauser R. M., Koffel J. N., Travis H. P., Dickinson P. J., 1975. - « Temporal change in occupational mobility : evidence for men in the United States », American sociological review, 40, 3, pp. 279-297.

Hout M., 1984. - «Status, autonomy, and training in occupational mobility », American journal of sociology, 89, 6, pp. 1379-1409.

- 1988. - «More universalism, less structural mobility : the American occupational structure in the 1980s », American journal of sociology, 93, 6, pp. 1358-1400.

Jones F. L., 1992. - «Common social fluidity : a comment on recent criticisms », European sociological review, 8, 3, pp. 233-237.

Jonsson J. O., Mills C., 1993. - «Social mobility in the 1970s and 1980s : a study of men and women in England and Sweden », European sociological review, 9, 3, pp. 229-248.

Kaelble H., 1981. - Historical research on social mobility: Western Europe and the USA in the nineteenth and twentieth centuries, New York, Columbia University Press.

Kerr C., Dunlop J. T., Harbinson F. H., Myers C. A., 1960. - Industrialism and industrial man: the problems of labor and management in economic growth, Cambridge, Harvard University Press [2" éd. 1973].

Kurz K., Muiller W., 1987. - «Class mobility in the industrial world », Annual review of sociology, 13, pp. 417-442.

Luijkx R., 1994. - «False and correct interpretations of structural mobility parameters » dans R. Luijkx, Comparative loglinear analyses of social mobility and heterogamy, Tilburg, Tilburg University Press, pp. $211-232$.

Luijkx R., Ganzeboom H. B. G., 1989. - «Intergenerational class mobility in the Netherlands between 1970 and 1985. Structural composition, structural differences and relative mobility » dans 
W. Jansen, J. Dronkers, K. Verrips (eds.), Similar or different? Continuities in Dutch research on social stratification and social mobility, Amsterdam, siswo, pp. 5-30.

Mendras H., 1988. - La Seconde Révolution française, 1965-1984, Paris, Gallimard.

Merllié D., 1994. - Les enquêtes de mobilité sociale, Paris, Presses Universitaires de France.

Merllié D., Prévot J., 1997. - La mobilité sociale, Paris, La Découverte [1 ${ }^{\text {ìre }}$ éd. 1991 ].

Miles A., Vincent D. (eds.), 1993. - Building European society. Occupational change and social mobility in Europe, 1840-1940, Manchester, Manchester University Press.

Parsons T., 1951. - The social system, Glencoe, Free Press.

Raftery A. E., 1986. - «Choosing models for cross-classifications », American sociological review, 51 , 1, pp. 145-146.

- 1995. - «Bayesian model selection in social research», Sociological methodology, 25, pp. 111-163.

Sewell W. H. Jr, 1985. - Structure and mobility. The men and women of Marseille, 1820-1870, Cambridge, Paris, Cambridge University Press, Éditions de la Maison des Sciences de l'Homme.

Seys B., 1984. - « De l'ancien code à la nouvelle nomenclature des catégories socioprofessionnelles », Économie et statistique, 171-172, pp. 159-166.

Sorokin P. A., 1927. - Social mobility, New York, Harper \& Brothers [rééd. en 1959, Social and cultural mobility, Glencoe, Free Press].

Thélot C., 1982. - Tel père, tel fils ? Position sociale et origine familiale, Paris, Dunod.

- 1983. - «L'évolution de la mobilité sociale dans chaque génération », Économie et statistique, 161, pp. 3-21.

Treiman D. J., 1970. - «Industrialization and social stratification » dans E. O. Laumann (ed.), Social stratification : research and theory for the 1970's, Indianapolis, Bobbs-Merrill, pp. 207-234.

Vallet L.-A., 1986. - «Activité professionnelle de la femme mariée et détermination de la position sociale de la famille. Un test empirique : la France entre 1962 et 1982 », Revue française de sociologie, 27, 4, pp. 655-696.

- 1988. - «L'évolution de l'inégalité des chances devant l'enseignement. Un point de vue de modélisation statistique », Revue française de sociologie, 29, 3, pp. 395-423.

- 1991. - La mobilité sociale des femmes en France. La participation des femmes aux processus de mobilité sociale intergénérationnelle, Thèse de doctorat de l'Université de Paris-Sorbonne, sous la direction de Raymond Boudon.

- 1992. - «La mobilité sociale des femmes en France. Principaux résultats d'une recherche » dans L. Coutrot, C. Dubar (éd.), Cheminements professionnels et mobilités sociales, Paris, La Documentation Française, pp. 179-200.

Van Leeuwen M. H. D., Maas I., 1996. - «Long-term social mobility : research agenda and a case study (Berlin, 1825-1957)», Continuity and change, 11, 3, pp. 399-433.

Vermunt J. K., 1997. - Log-linear models for event histories, London, Sage.

Weakliem D. L., 1992. - «Comparing non-nested models for contingency tables», Sociological methodology, 22, pp. 147-178.

Wong R. S.-K., 1994. - «Postwar mobility trends in advanced industrial societies », Research in social stratification and mobility, 13, pp. 121-144.

- 1995. - «xtensions in the use of log-multiplicative scaled association models in multiway contingency tables », Sociological methods \& research, 23, 4, pp. 507-538.

Wong R. S.-K., Hauser R. M., 1992. - «Trends in occupational mobility in Hungary under socialism », Social science research, 21,4 , pp. 419-444.

Xie Y., 1992. - «The log-multiplicative layer effect model for comparing mobility tables », American sociological review, 57,3 , pp. 380-395. 